The Production of High $p_{T} \pi^{0}$ Mesons in $515 \mathrm{GeV} / \mathrm{c} \pi^{-}-$Nucleus Collisions

A dissertation presented

by

Wieslaw Dlugosz

to

The Department of Physics

In partial fulfillment of the requirements

for the degree of

Doctor of Philosophy

in the field of

Physics

Northeastern University

Boston, Massachusetts

August 1994

\title{
FERMILAB LIBRARY
}


$-$

$-$

$-$

$-$

$-$

$-$ 
The Production of High $p_{T} \pi^{0}$ Mesons

in $515 \mathrm{GeV} / \mathrm{c} \pi^{-}-$Nucleus Collisions

by

Wieslaw Dlugosz

The inclusive production cross sections of $\pi^{0}$ mesons from a $515 \mathrm{GeV} / \mathrm{c} \pi^{-}$beam incident on $\mathrm{Be}$ and $\mathrm{Cu}$ have been measured as functions of transverse momentum $p_{T}$ and rapidity $y$. The data were collected during the 1990 run of experiment E706 at Fermilab. E706 triggered on events containing high $p_{T}$ electromagnetic showers detected in a finely segmented lead liquid argon calorimeter. The spectrometer also included a charged particle tracking system and momentum analysis magnet upstream of the calorimeter. $\pi^{0}$ mesons were reconstructed via the photons detected in the electromagnetic calorimeter. Results are reported in the rapidity range $-0.75<y<0.75$ and in the transverse momentum range $4.0<p_{T}<12.0 \mathrm{GeV} / \mathrm{c}$ for $\mathrm{Be}$ and $4.0<p_{T}<10.0 \mathrm{GeV} / \mathrm{c}$ for $\mathrm{Cu}$. Measurements are compared with current next-to-leading logarithm QCD calculations and data from other experiments. The nuclear dependence of the $\pi^{0}$ production cross section is also discussed.

Submitted in partial fulfillment of the requirements for the degree of Doctor of Philosophy in Physics in the Graduate School of Arts and Sciences of Northeastern University, August 1994 
$-$ 


\section{ACKNOWLEDGEMENTS}

I would like to thank my thesis advisor, Professor David Garelick, and Professors Michael Glaubman, George Alverson and William Faissler for introducing me to the field of high energy physics and their support and encouragement during my stay at Fermilab.

Professors Tom Ferbel, Fred Lobkowicz and Paul Slattery provided me with helpful comments and advice for which I am thankful.

I am grateful to George Ginther, Takahiro Yasuda and Marek Zieliński for guidance and patience with my endless questions. Chris Lirakis and Dane Skow taught me about the DA, John Mansour made me familiar with the EMREC, Win Baker and Roger Tokarek showed me how to handle the beam. I express my gratitude to them.

Dan Ruggiero, Lenny Apanasevich, John Bacigalupi, David Brown, Michael Begel, Steve Blusk, Paoti Chang, Woohyun Chung, Lucy de Barbaro, Jim Dunlea, Vijay Kapoor, John Kuehler, Sudhindra Mani, Andre Maul, George Osborne, Ed Pothier, Rob Roser, Lee Sorrell, David Striley, Nikos Varelas, Dhammika Weerasundara, Carlos Yosef and Vishnu Zutshi made my work on E706 enjoyable and educational.

Before entering the field of the GeVs, I had an opportunity to study phenomena on the $\mathrm{eV}$ scale. Professor Arun Bansil in Boston and Professors Stanisław Kaprzyk and Andrzej Oleś in Kraków taught me about electronic properties of materials and showed me how to study solids on computer. Doctor Lucjan Pytlik spent many hours introducing me into the physics of the phase transitions. I am grateful to them for sharing their knowledge with me. 


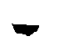

$-$

$-$ 


\section{Contents}

Abstract :

Acknowledgements

Contents $\quad$ v

List of Figures viii

List of Tables $\quad$ xii

1 Introduction 1

1.1 Hadron-Hadron Interactions in QCD . . . . . . . . . . 2

1.2 Nuclear Effects in Hadron-Hadron Interactions . . . . . . . . . 10

1.3 Other Experiments . . . . . . . . . . . . . 10

2 The E706 Spectrometer 13

2.1 The Meson West Beamline . . . . . . . . . . . . . 15

2.2 The Target Region $\ldots \ldots \ldots \ldots \ldots \ldots \ldots$

2.3 The Tracking System . . . . . . . . . . . . . . . . 19

2.3.1 The Silicon Strip Detectors . . . . . . . . . . . . 19

2.3.2 The Analysis Magnet . . . . . . . . . . . . . . . 21

2.3.3 The Proportional Wire Chambers and Straw Drift Tubes . . 22

2.4 Calorimetry . . . . . . . . . . . . . . . 23 
2.4.1 The Liquid Argon Calorimeter . . . . . . . . . . . . 23

2.4.2 The Forward Calorimeter $\ldots \ldots \ldots \ldots \ldots \ldots$

3 The E706 Trigger and Data Acquisition System 33

3.1 Overview. . . . . . . . . . . . . . . 33

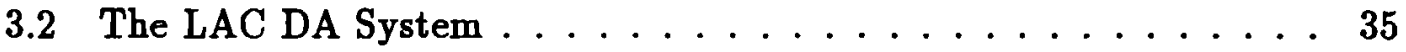

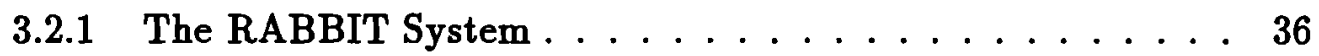

3.2 .2 The FASTBUS System $\ldots \ldots \ldots \ldots \ldots \ldots$

3.3 The E706 Trigger $\ldots \ldots \ldots \ldots \ldots \ldots \ldots \ldots$

3.4 Event Readout . . . . . . . . . . . . . . . . . 49

3.4.1 LAC Event Readout Tasks . . . . . . . . . . . . . 51

4 Event Reconstruction $\quad 56$

4.1 DLREC $\ldots \ldots \ldots \ldots \ldots \ldots \ldots \ldots \ldots$

$4.2 \quad$ PLREC $\ldots \ldots \ldots \ldots \ldots \ldots \ldots \ldots \ldots$

4.3 EMREC and HCREC $\ldots \ldots \ldots \ldots \ldots \ldots$

4.3.1 The Electromagnetic Shower Shape . . . . . . . . . . 61

4.3.2 The EMREC Algorithm $\ldots \ldots \ldots \ldots \ldots$

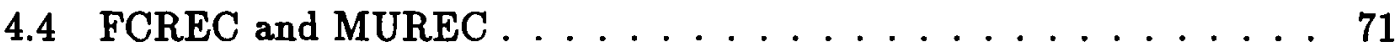

5 Data Analysis $\quad 73$

5.1 Data Selection . . . . . . . . . . . . . . . . 76

5.1 .1 The Vertex Cut ............... 76

5.1.2 The EMLAC Fiducial Volume . . . . . . . . . 76

5.1.3 The Energy Asymmetry . . . . . . . . . . . 81

5.1.4 Hadron Rejection . . . . . . . . . . . . . . 81

5.1.5 Muon Rejection . . . . . . . . . . . . . . 83

5.1.6 The $\pi^{0}$ Signal Definition . . . . . . . . . . 90 
5.2 The Monte Carlo Simulation . . . . . . . . . . . . . . . . 93

5.2.1 Reconstruction Efficiency . . . . . . . . . . . 96

5.3 Trigger Corrections . . . . . . . . . . . . . . . 97

5.4 Beam Normalization and Energy . . . . . . . . . . . 101

5.5 The Cross Section Definition . . . . . . . . . . . . . . 102

6 Results $\quad 105$

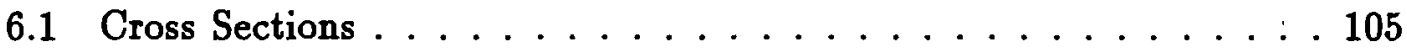

6.2 Comparisons with Other Experiments . . . . . . . . . 113

6.3 Nuclear Dependence . . . . . . . . . . . . . . 113

6.4 Comparison with Theoretical Predictions . . . . . . . . . . 113

6.5 Major Sources of Systematic Uncertainties . . . . . . . . . . 117

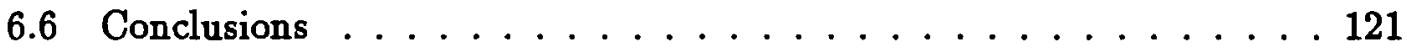

$\begin{array}{lr}\text { Bibliography } & 122\end{array}$

$\begin{array}{ll}\text { Resume } & \mathbf{1 2 7}\end{array}$ 


\section{List of Figures}

1.1 Elementary vertices of QCD. . . . . . . . . . . . 4

1.2 Top: A hadron hadron collision. Bottom: A hadron nucleus collision. 7

2.1 Layout of the MWEST spectrometer. . . . . . . . . . . . 14

2.2 The Meson West beamline. . . . . . . . . . . . . . 16

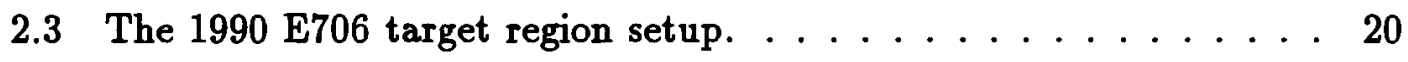

2.4 A side view of the LAC. . . . . . . . . . . . . . 24

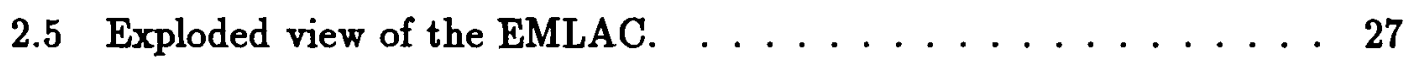

2.6 Structure of the HALAC cell. . . . . . . . . . . . . . . 30

2.7 Geometry of the HALAC readout pads. . . . . . . . . . . . 31

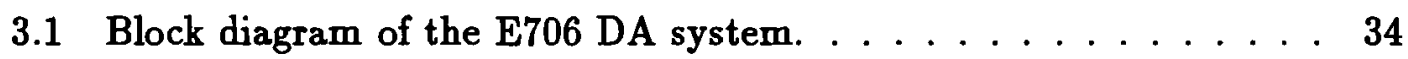

3.2 The LAC DA system. . . . . . . . . . . . . . . . 37

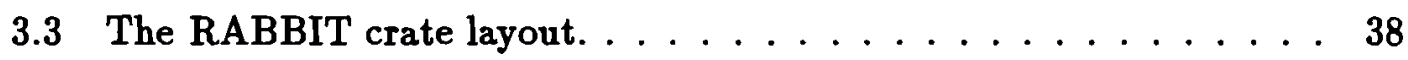

3.4 Block diagram of the LACAMP card. . . . . . . . . . . . 39

3.5 The ICBM block diagram. . . . . . . . . . . . . . 44

3.6 Local trigger signal formation. . . . . . . . . . . . . . 46

5.1 The two photon invariant mass spectrum for $p_{T}>4.0 \mathrm{GeV} / \mathrm{c}$ and $-0.75<y<0.75$. Cuts described in this chapter have been applied to obtain this distribution. . . . . . . . . . . 75 
5.2 Unweighted distributions of the $\pi^{0}$ event vertices. a) The distribution along the $\mathrm{z}$ coordinate axis. b) The $\mathrm{x}-\mathrm{y}$ distribution in the Cu targets. c) The $x-y$ distribution in the Be targets. . . . . . 77

5.3 The distribution of the $\pi^{0}$ candidates within the EMLAC fiducial

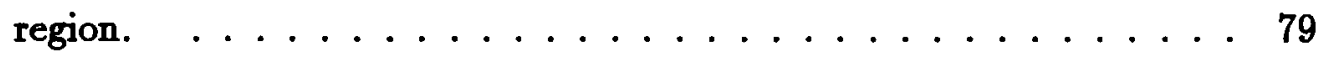

5.4 The averaged $\pi^{0}$ geometric acceptance function in selected $p_{T}$ bins. $\quad 80$

5.5 Top: The definition of the energy asymmetry. Bottom: The asymmetry spectrum of the reconstructed $\pi^{0} \mathrm{~s} \ldots \ldots \ldots \ldots$. . . . 82

5.6 $\pi^{0}$ mass distributions after applying $E_{f} / E_{t}$ and asymmetry cuts. . 84

5.7 The impact of the veto wall and the muon rejection cuts on the $\pi^{0}$ mass distribution for $7.0<p_{T}<9.0 \mathrm{GeV} / \mathrm{c}$. Top row: distributions after $E_{f} / E_{t}$ and asymmetry cuts. Middle row: impact of the veto wall cut. Bottom row: impact of the additional muon rejection cuts. 85

5.8 Photon directionality distributions in rapidity bins for $\pi^{0}$ with $5.5<$ $p_{T}<9.0 \mathrm{GeV} / \mathrm{c}$. Left column: events with the off-line veto wall signal. Right column: Events without the off-line veto wall signal. . 86

5.9 Balanced $p_{T}$ distributions for $\pi^{0}$ with $5.5<p_{T}<9.0 \mathrm{GeV} / \mathrm{c}$. Left column: events with off-line veto wall signal. Right column: Events without off-line veto wall signal. . . . . . . . . . . . . 87

5.10 Photon scaled $\chi^{2}$ distribution for $\pi^{0}$ with $5.5<p_{T}<9.0 \mathrm{GeV} / \mathrm{c}$. Left column: events with off-line veto wall signal. Right column: Events without off-line veto wall signal. . . . . . . . 88

$5.11 \pi^{0}$ and $\eta$ mass distributions after the analysis cuts. . . . . . . 91

$5.12 \pi^{0}(\bullet)$ and $\eta(\circ)$ masses as functions of the octant number, $p_{T}$, and radial position. Masses are normalized to the world averages. . . . 92 
5.13 Top: $\pi^{0}$ candidates mass distributions from data (histogram) and Monte Carlo simulation (o). Bottom: $\pi^{0}$ candidates energy asymmetry distributions from data (histogram) and Monte Carlo simulation (o). Histograms are area normalized. . . . . . . . . . 94

5.14 Photon $E_{f} / E_{t}$ distributions from the data (histogram) and the Monte Carlo simulation (o) in selected photon energy bins. Histograms are area normalized. . . . . . . . . . . . . . . 95

5.15 The $\pi^{0}$ reconstruction efficiency as a function of $p_{T}$ and y. . . . 98

5.16 Trigger efficiency curves for SLH (solid line) and SLL (dashed line) triggers for selected sections in the inner (top) and outer (bottom) sections of an octant as functions of the trigger $p_{T} . \ldots \ldots \ldots 99$

5.17 PRETRIGGER efficiency curves for the inner (left column) and outer (right column) sections of an octant as functions of the trigger

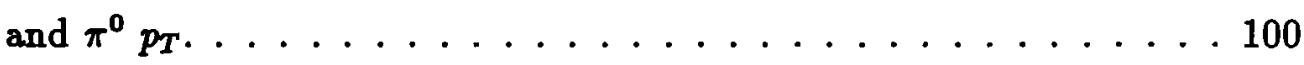

6.1 The $p_{T}$ dependence of the $\pi^{0}$ invariant production cross section per nucleon on a Be target. The dashed line is a fit to Equation 6.1. $\quad .106$

6.2 The $p_{T}$ dependence of the $\pi^{0}$ invariant production cross section per nucleon on a $\mathrm{Cu}$ target. The dashed line is a fit presented in Figure

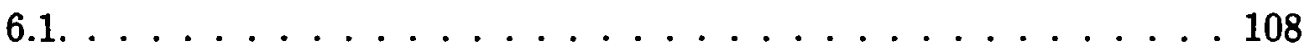

6.3 The $p_{T}$ dependence of the $\pi^{0}$ invariant production cross section per nucleon on a Be target in selected $(y \leq 0)$ rapidity bins. . . . . 110

6.4 The $p_{T}$ dependence of the $\pi^{0}$ invariant production cross section per nucleon on a $\mathrm{Be}$ target in selected $(y \geq 0)$ rapidity bins. . . . . 111

6.5 The rapidity dependence of the $\pi^{0}$ invariant production cross section per nucleon on a Be target in selected $p_{T}$ bins. . . . . . . 112 
6.6 Comparison of the E706 $\pi^{0}$ invariant cross section on a Be target to the results from other experiments. Results of the experiments E706 and NA3 have been corrected for nuclear dependence (see Section

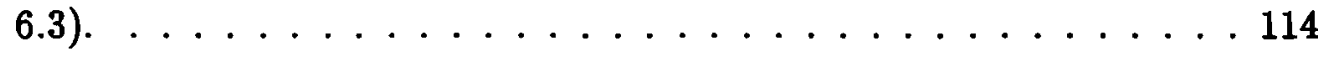

6.7 a) The $p_{T}$ dependence of $\alpha$. b), c), d) The rapidity dependence of $\alpha$ in selected $p_{T}$ bins. . . . . . . . . . . . 115

6.8 The $p_{T}$ dependence of $\alpha$ for $\pi^{-}, \pi^{+}$and $\pi^{0}$ production. Charged pion data are from Reference 26. . . . . . . . . . . . . 116

6.9 Comparison of the $\pi^{0}$ invariant cross section per nucleon on a $\mathrm{Be}$ target with the NLL calculations. . . . . . . . . . . . 118

6.10 Comparison of the $\pi^{0}$ invariant cross section per nucleon on a $\mathrm{Cu}$ target with the NLL calculations. . . . . . . . . . . 119 


\section{List of Tables}

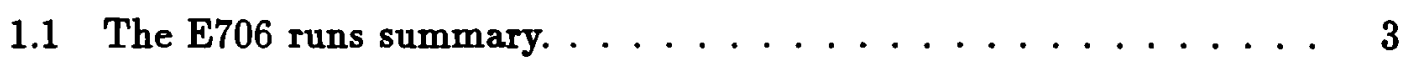

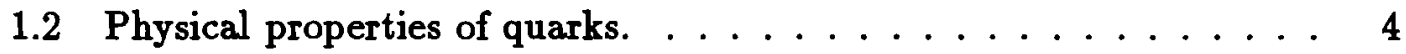

1.3 Characteristics of selected fixed target experiments measuring high $p_{T}$ pion cross sections using a $\pi^{-}$beam. . . . . . . . 11

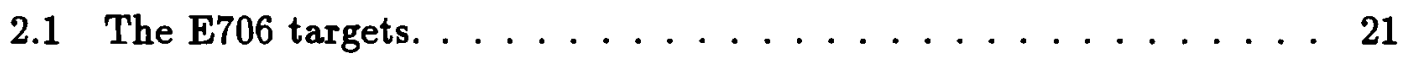

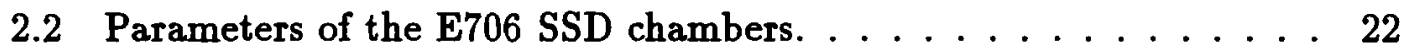

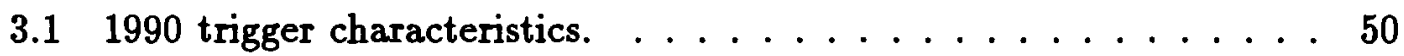

4.1 The simplest EMREC correlation types. Types $15-18$ correspond to $11-14$, but two peaks are created after adding the views separated by the view boundary. In types $21-24$ the boundary photon

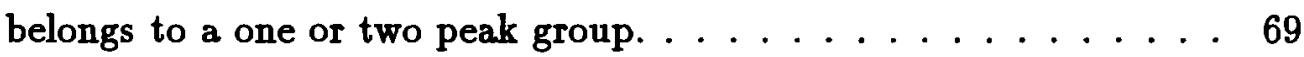

4.2 Multiple gamma correlation types defined in the EMREC. . . . . 70

5.11990 trigger sets. . . . . . . . . . . . . . . . . . 74

5.2 Summary of the averaged corrections. . . . . . . . . . . 104

6.1 The $\pi^{0}$ invariant cross section per nucleon on a Be target. . . . . 107

6.2 The $\pi^{0}$ invariant cross section per nucleon on a $\mathrm{Cu}$ target. . . . . 109

6.3 Values of $\alpha$ averaged over the rapidity range $-0.75<y<0.75 \ldots 117$ 


\section{Chapter 1}

\section{Introduction}

The study of the particles produced at high transverse momenta (high $p_{T}$ ) in hadronic interactions provides a method of determining the parton structure of hadrons. Deep inelastic scattering (DIS) experiments helped establish the parton model and measure quark structure functions, but hadron-hadron scattering with the production of jets and direct photons is a direct probe of the gluonic component of strongly interacting particles, as well as their quark content $[1,2]$. (Direct photons are photons that are produced in the primary scattering, as opposed to photons from hadron decays.) $\Lambda_{Q C D}$, the characteristic energy scale of the strong interaction which is approximately $200 \mathrm{MeV}$, is small compared to the collision energy scale in events with high $p_{T}$, so perturbative Quantum Chromodynamics ( $\mathrm{PQCD}$ ) can be applied and its predictions can be checked against the measured production cross sections of high $p_{T}$ jets, single hadrons and direct photons.

Fermilab fixed target experiment E706 was designed to perform precise measurements of high $p_{T}$ phenomena in hadron-nucleus and hadron-proton interactions. Its main components were a finely segmented liquid argon calorimeter and a sophisticated charged particle tracking system. The first run of E706 was completed in 1988 and results from it have been published since $[3,4,5,6]$. Between 1988 and 1990 several improvements were made to the apparatus, and the pattern 
recognition software was upgraded. This enabled us to collect an order of magnitude more, as well as better, data in runs in 1990 and in 1991 (Table 1.1). In this thesis, I will concentrate on the measurements of high $p_{T} \pi^{0}$ meson cross sections on two nuclear targets, Be and $\mathrm{Cu}$. These $\pi^{0}$ 's are usually the leading particles of the jets produced in the collision, i.e., their momentum is the closest to that of the originally produced parton. Measured cross sections provide material for the extraction as well as tests of the $\pi^{0}$ fragmentation functions. They can also be used to study the influence of the nuclear medium on the parton propagation and parton structure functions.

The rest of this chapter contains an overview of the underlying physics. Chapter 2 describes the E706 spectrometer, while Chapters 3 and 4 are devoted to the data acquisition system and event reconstruction procedures. Chapter 5 presents the data analysis and Chapter 6 contains the results and conclusions.

\subsection{Hadron-Hadron Interactions in QCD}

Quantum Chromodynamics, one of the components of the Standard Model of particle physics, describes the interactions and the structure of hadrons in the language of a gauge field theory $[7,8]$. It is based on an unbroken, non-Abelian gauge group SU(3). Its elementary interaction vertices between quarks (spin 1/2 fermions) and gluons (massless spin 1 gauge bosons) are presented in Figure 1.1 below.

The mass estimates and electric charges of the six quark flavors are listed in Table $1.2[9]$.

Both quarks and gluons carry an additional quantum number, color, analogous to the electric charge in QED. All known bound states of a quark $q$ and an antiquark $\bar{q}$ (mesons), or three quarks (baryons), are color neutral. For example, the quark 


\begin{tabular}{|c|c|c|c|c|}
\hline Run & Interaction & $\begin{array}{c}\text { Beam Momentum } \\
(\mathrm{GeV} / \mathrm{c})\end{array}$ & Number of Events & $\begin{array}{l}\text { Sensitivity } \\
\text { (events/pb) }\end{array}$ \\
\hline 1988 & $\begin{array}{c}\pi^{-} \mathrm{Be} \\
\pi^{-} \mathrm{Cu} \\
\left(\mathrm{p}, \pi^{+}\right) \mathrm{Be} \\
\left(\mathrm{p}, \pi^{+}\right) \mathrm{Cu}\end{array}$ & 500 & $\begin{array}{l}2 \times 10^{6} \\
3 \times 10^{6}\end{array}$ & $\begin{array}{l}0.5 \\
0.1 \\
0.75 \\
0.1\end{array}$ \\
\hline 1990 & $\begin{array}{l}\pi^{-\mathrm{Be}} \\
\pi^{-\mathrm{Cu}}\end{array}$ & 515 & $30 \times 10^{6}$ & $\begin{array}{l}8.6 \\
1.4\end{array}$ \\
\hline \multirow{3}{*}{1991} & $\begin{array}{c}\mathrm{pBe} \\
\mathrm{pCu} \\
\mathrm{pH}\end{array}$ & 800 & $23 \times 10^{6}$ & $\begin{array}{l}7.3 \\
1.8 \\
1.5\end{array}$ \\
\hline & $\begin{array}{c}\left(\mathrm{p}, \pi^{+}\right) \mathrm{Be} \\
\left(\mathrm{p}, \pi^{+}\right) \mathrm{Cu} \\
\left(\mathrm{p}, \pi^{+}\right) \mathrm{H}\end{array}$ & 515 & $14 \times 10^{6}$ & $\begin{array}{l}6.4 \\
1.6 \\
1.3\end{array}$ \\
\hline & $\begin{array}{c}\pi^{-} \mathrm{Be} \\
\pi^{-} \mathrm{Cu} \\
\pi^{-} \mathrm{H}\end{array}$ & 515 & $4 \times 10^{6}$ & $\begin{array}{l}1.4 \\
0.3 \\
0.3\end{array}$ \\
\hline
\end{tabular}

Table 1.1: The E706 runs summary. 

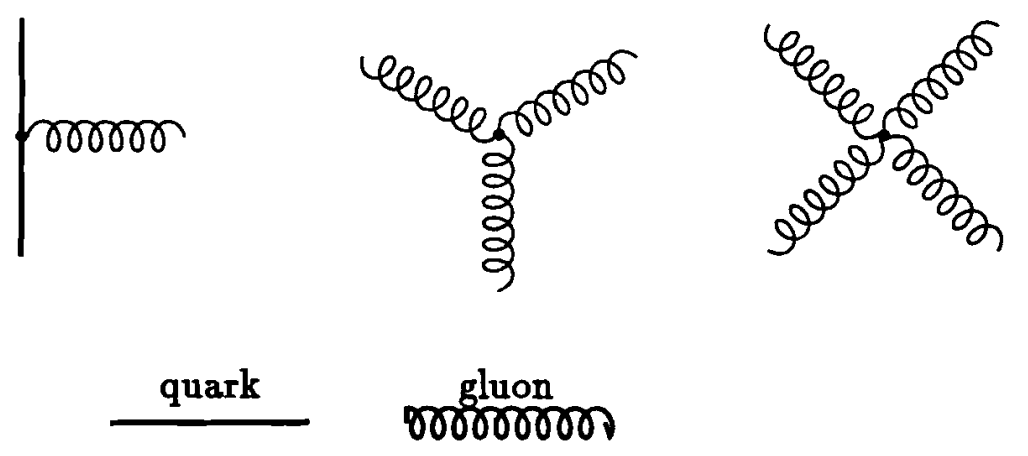

Figure 1.1: Elementary vertices of QCD.

model composition of the $\pi^{0}$ and $\eta$ mesons are:

$$
\begin{gathered}
\pi^{0}=(d \bar{d}-u \bar{u}) / \sqrt{2} \\
\eta=\eta_{8} \sin \theta_{P}+\eta_{0} \cos \theta_{P}
\end{gathered}
$$

where $\eta_{8}$ and $\eta_{0}$ are defined as:

$$
\eta_{8}=(d \bar{d}+u \bar{u}-2 s \bar{s}) / \sqrt{6} \quad \eta_{0}=(d \bar{d}+u \bar{u}+s \bar{s}) / \sqrt{3}
$$

and $\theta_{P}$ is the $\eta_{0}-\eta_{8}$ mixing angle $[10]$.

For the lightest baryons, the proton and the neutron, we have:

\begin{tabular}{|l|c|c|}
\hline $\begin{array}{l}\text { Quark } \\
\text { Flavour }\end{array}$ & $\begin{array}{c}\text { Electric } \\
\text { Charge }(\mathrm{e})\end{array}$ & $\begin{array}{c}\text { Mass Estimate } \\
\left(\mathrm{GeV} / \mathrm{c}^{2}\right)\end{array}$ \\
\hline \hline$d$ (down) & $-1 / 3$ & $0.0099 \pm 0.0011$ \\
$u$ (up) & $+2 / 3$ & $0.0056 \pm 0.0011$ \\
\hline \hline$s$ (strange) & $-1 / 3$ & $0.199 \pm 0.033$ \\
$c$ (charm) & $+2 / 3$ & $1.35 \pm 0.05$ \\
\hline \hline$b$ (bottom) & $-1 / 3$ & $\sim 5$ \\
$t$ (top) & $+2 / 3$ & $174 \pm 10$ \\
\hline
\end{tabular}

Table 1.2: Physical properties of quarks. 


$$
p=u u d \quad n=u d d
$$

The fact that gluons are colored accounts for the self-interaction of these gauge bosons, which leads to the prediction of glueballs, bound states of gluons, as well as to the phenomenon of asymptotic freedom, the decrease in the strength of the interaction with increasing energy scale $Q^{2}$ of the interaction $[11,12]$. The divergences of the theory are handled by renormalization. This leads to a dependence of the strong coupling constant $\alpha$, on $Q^{2}$ which is given in the leading logarithm (LL) approximation by:

$$
\alpha_{s}\left(Q^{2}\right)=\frac{12 \pi}{\left(33-2 n_{f}\right) \ln \left(Q^{2} / \Lambda_{Q C D}^{2}\right)}
$$

$n_{f}$ is the number of quark flavors and $\Lambda_{Q C D}$ is a QCD scale parameter defined by:

$$
\ln \left(\Lambda_{Q C D}^{2}\right)=\ln \left(\mu^{2}\right)-\frac{12 \pi}{\left(33-2 n_{f}\right) \alpha_{s}\left(\mu^{2}\right)}
$$

$\mu$ being the renormalization point. The experimental value of $\alpha_{s}$ is 0.12 at the mass of the $Z$ boson $(91 \mathrm{GeV})$ and 0.35 at the mass of the $\tau(1.8 \mathrm{GeV})$ [13]. Since this coupling is relatively weak at large $Q^{2}$, one can apply perturbation methods to the QCD Lagrangian in order to calculate cross sections for processes characterised by large momentum transfers between the partons of the scattering hadrons, which in the case treated in this thesis results in the high $p_{T}$ production of $\pi^{0}$ 's. At small energies, or distances large in comparison with the range of the color force, $\alpha_{s}$ is large, and nonperturbative methods are needed to describe physical phenomena [14].

Perturbative QCD applied to the high energy hadron-hadron scattering processes allows for a simple visualization of such phenomena [15]. If we are interested in the measurement of the inclusive production cross section of a specific hadron $C:$ 


$$
A+B \rightarrow C+X
$$

where $A$ and $B$ are colliding hadrons and $X$ stands for the remaining products, the pQCD formula for the invariant cross section $E_{C} d^{3} \sigma / d p_{C}^{3}$ can be written as:

$$
\begin{array}{r}
E_{C} \frac{d^{3} \sigma}{d p_{C}^{3}}(A+B \rightarrow C+X)=\sum_{a b c d} \int d x_{a} d x_{b} d z_{c} \delta(\hat{s}+\hat{t}+\hat{u}) \frac{\hat{s}}{z_{c}^{2} \pi} \\
\quad \times G_{a / A}\left(x_{a}, M_{d}^{2}\right) G_{b / B}\left(x_{b}, M_{d}^{2}\right) \frac{d \sigma}{d \hat{t}}(a b \rightarrow c d) D_{C / c}\left(z_{c}, M_{f}^{2}\right)
\end{array}
$$

$\frac{d \sigma}{d \hat{t}}(a b \rightarrow c d)$ is the parton level hard scattering cross section, and $\hat{s}, \hat{t}$ and $\hat{u}$ are the parton level Mandelstam variables. $p_{A}, p_{B}, p_{C}$ are the hadron momenta, and $p_{a}=$ $x_{a} p_{A}, p_{b}=x_{b} p_{B}, p_{c}=p_{C} / z_{c}$ are the parton momenta. To lowest order (scaling) the probability of the parton carrying fraction $x$ of the hadron's momentum, the structure function, is independent of $Q^{2}$, but the renormalization and the need to be able to use independent structure functions for each hadron (factorization), introduces an energy scale and $Q^{2}$ dependence which for the structure functions $G$ is $M_{d}^{2}$ and for the fragmentation function $D$ is $M_{f}^{2}$. The integral is a convolution of these functions with the parton level cross section. More will be said about the $M^{2}$ 's later in this chapter; the outstanding feature of this formula is that $G_{a / A}\left(x_{a}, M_{d}^{2}\right)$, the probability of finding parton $a$ with fraction $x_{a}$ of the momentum of hadron $A$, correspondingly $G_{b / B}$, as well as $D_{C / c}\left(z_{c}, M_{f}^{2}\right)$, the probability of finding hadron $C$ with the fraction $z_{c}$ of the momentum of the outgoing parton $c$, all enter into the convolution independently. Formulas similar to Equation 1.6 can be written for a variety of scattering processes and are know as factorization theorems. Equation 1.6 is shown schematically in the upper section of Figure 1.2.

Parton distribution functions and hadron fragmentation functions characterize the nonperturbative (long range) aspects of QCD. At present they cannot be obtained from theory and have to be extracted from experiment. These functions 

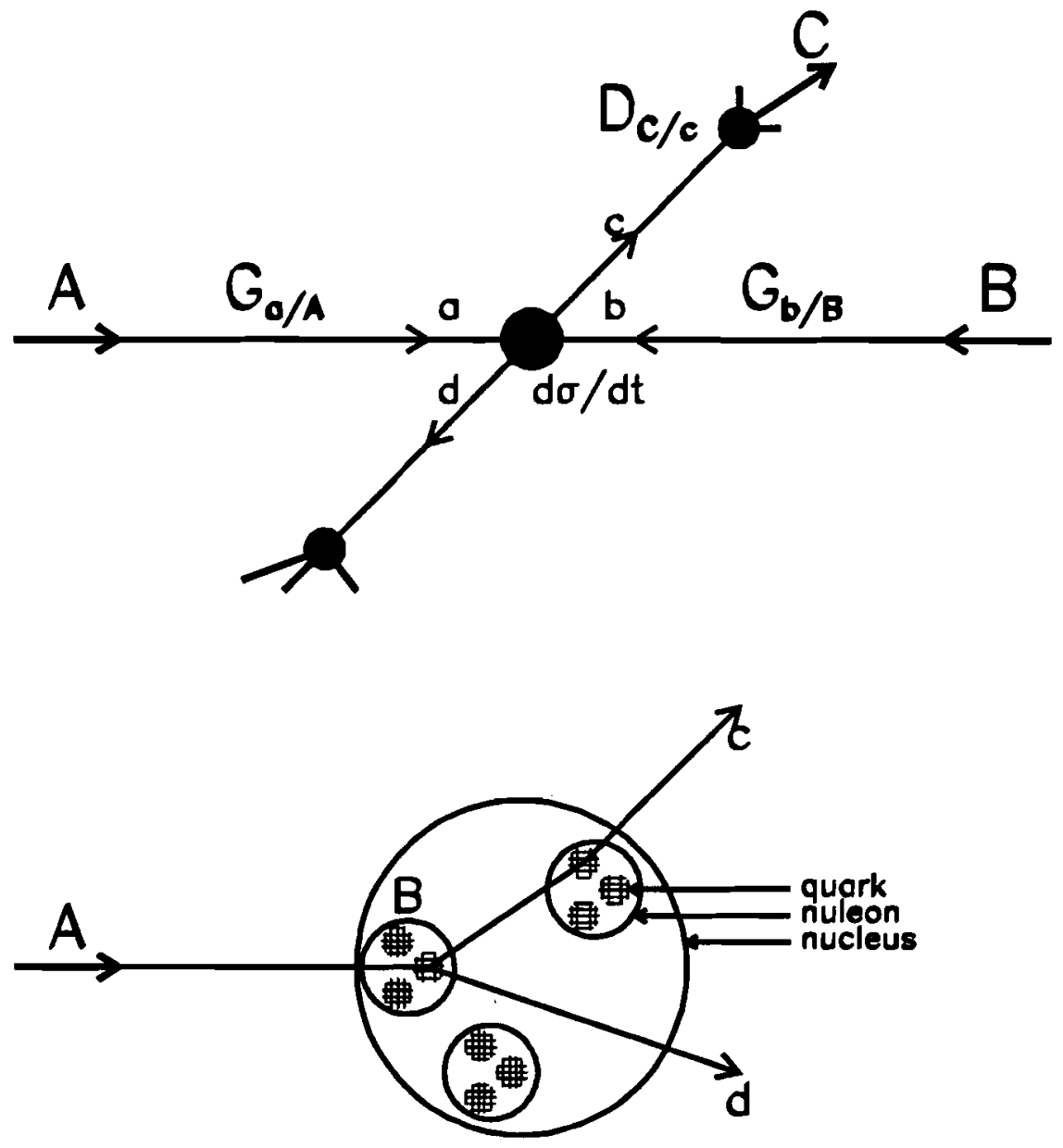

Figure 1.2: Top: A hadron hadron collision. Bottom: A hadron nucleus collision. 
are presumed to be universal in the sense that quark distribution functions obtained from DIS experiments and fragmentation functions determined from $e^{+} e^{-}$ annihilation can be used to make predictions in the case of hadronic reactions. Parametrizations of distribution and fragmentation functions are obtained from global fits to the results of complementary experiments within the framework of PQCD [16]. As hard hadronic processes involve interactions between all the partons in the scattering particles, the measurements of jet, single hadron and photon production cross sections provide important tests of currently available parametrizations and data that can be used to improve them.

Theoretical formulas used in the global fits may be derived using various renormalization schemes and various degrees of approximation. If we expand the formula for the cross section $\sigma$ of a parton level process in terms of powers of $\alpha_{s}$, i.e., beyond the single vertices in the diagrams of Figure 1.1 (see for example $[17,18]$ ), we obtain the following series:

$$
\sigma=\sigma_{0}\left(1+\alpha_{s} A_{1}+\alpha_{s}^{2}\left(A_{2} \ln Q^{2}+B_{2}\right)+\alpha_{s}^{3}\left(A_{3} \ln ^{2} Q^{2}+B_{3} \ln Q^{2}+C_{3}\right)+\ldots\right)
$$

$Q^{2}$ is the energy scale of the reaction in question and the value of $\alpha_{s}$ is determined at the renormalization point. The LL approximation is based on summing all the $A_{i}$ terms in the above expansion, the so called leading logarithms. Only tree level diagrams are used to calculate them and they do not depend on the renormalization scale which is introduced at LL only via $\alpha_{s}$. Inclusion of the less dominant $B_{i}$ terms leads to the next-to-leading logarithm approximation (NLL). Currently, the calculations of all the components needed to obtain the theoretical $\pi^{0}$ production cross section in NLL are available [19]. To find the parton-parton hard scattering cross sections, $O\left(\alpha_{s}^{3}\right)$ matrix elements were used. Being functions of the renormalization scale, their presence compensates partially for the renormalization scale dependence introduced by the $\alpha_{s}[20]$. Two loop evolved distribution $[21,22]$ and 
fragmentation [23] functions were also used. Such NLL hadronic cross section can be written as:

$$
\sigma_{\pi^{0}}=\alpha_{s}^{2} A+\alpha_{s}^{3}\left[2 A \ln \left(\frac{Q^{2}}{\Lambda^{2}}\right)+B \ln \left(\frac{M_{d}^{2}}{\Lambda^{2}}\right)+C \ln \left(\frac{M_{f}^{2}}{\Lambda^{2}}\right)+D\right]
$$

The functions $A, B, C$ and $D$ depend on $M_{d}$ and $M_{f}$ via the distribution and fragmentation functions. The use of renormalization schemes in order to make theoretical predictions finite, and factorization of the cross sections introduce three independent energy scales:

- $Q^{2}$ defines the characteristic energy scale of the scattering process. For high $p_{T}$ hadronic processes it can be chosen as const $\times p_{T}^{2}$ and fitted to get the best agreement between data and theory. The $Q^{2}$ dependence of the strong coupling constant is governed by the renormalization group equation [8].

- $M_{d}^{2}$ and $M_{f}^{2}$ are distribution and fragmentation energy scales which result from incorporating processes not included in the parton level cross sections into the distribution and fragmentation functions. The $M$ dependence of the $G$ and $D$ functions is described by the Altarelli-Parisi equations [24].

One way of choosing appropriate scales is to put some requirements on the behavior of the cross section or higher order terms with respect to, for example $Q^{2}$, and solve the resulting equations for scale. It can be done only on the NLL (or higher) level. The conventional choice is to use the same definition for all scales and, as mentioned above, find one giving the best agreement with the data by trial and error. 


\subsection{Nuclear Effects in Hadron-Hadron Interac- tions}

Measurements of hadron production cross sections on nuclear targets provide an opportunity to study the behavior of partons traversing nuclear matter. The nuclear dependence of the cross section per nucleus $\left(\sigma_{A}\right)$ can be parametrized as:

$$
\sigma_{A}=\sigma A=\sigma_{0} A^{\alpha}
$$

where $\mathrm{A}$ is atomic number of the target material, $\sigma$ is the cross section per nucleon and $\sigma_{0}$ is a constant. For low $p_{T}$ collisions, the inelastic cross sections are proportional to $A^{2 / 3}$, the area shadowed by the nucleus which has a radius proportional to $A^{1 / 3}$. For high $p_{T}$ events, in which hard scattering between constituent partons dominate, it was found [25] that the power $\alpha$ is $p_{T}$ dependent. For $p_{T}>2 \mathrm{GeV} / \mathrm{c}$ the value of $\alpha$ exceeds 1 . This can be attributed to the rescattering of the partons in nuclear matter (see the bottom part of Figure 1.2). Comparison of the $\alpha$ obtained for hadrons and the one for direct photons may show whether incoming or outgoing partons are rescattered since direct photons are not likely to scatter because of the relative weakness of the electromagnetic coupling.

The parameter $\alpha$ can be found by measuring particle production cross sections on different nuclear targets (on $\mathrm{Be}$ and $\mathrm{Cu}$ for E706) and using the following formula derived from Equation 1.11:

$$
\alpha=1+\frac{\ln \left(\sigma_{C u} / \sigma_{B e}\right)}{\ln \left(A_{C u} / A_{B e}\right)}
$$

\subsection{Other Experiments}

The first observation of high- $p_{T}$ particle production in high-energy hadronic collisions was made at the CERN Intersecting Storage Rings (ISR). A broad review 


\begin{tabular}{|c|c|c|c|c|}
\hline \hline Experiment & Target & $\begin{array}{c}\sqrt{s} \\
(\mathrm{GeV})\end{array}$ & $\begin{array}{c}p_{T} \text { Range } \\
(\mathrm{GeV} / \mathrm{c})\end{array}$ & c.m. Rapidity Range \\
\hline E706 & $\mathrm{Be}, \mathrm{Cu}, \mathrm{H}_{2}$ & 31.1 & $1 \rightarrow 12$ & $-0.75 \rightarrow 0.75$ \\
E705 & $\mathrm{Li}$ & 23.7 & $4 \rightarrow 7$ & $-0.6 \rightarrow 0.8$ \\
E258 & $\mathrm{Be}, \mathrm{Cu}, \mathrm{W}, \mathrm{H}$ & $19.4,23.7$ & $1 \rightarrow 6$ & $\sim 0$ \\
E111 & $\mathrm{H}$ & $13.7,19.4$ & $1 \rightarrow 5$ & $\sim 0$ \\
\hline \hline WA70 & $\mathrm{H}_{2}$ & 22.9 & $4 \rightarrow 7$ & $-1.0 \rightarrow 1.3$ \\
NA24 & $\mathrm{H}_{2}$ & 23.7 & $1 \rightarrow 7$ & $-0.65 \rightarrow 0.52$ \\
NA3 & $\mathrm{C}$ & 19.4 & $3 \rightarrow 6$ & $-0.4 \rightarrow 1.2$ \\
\hline \hline
\end{tabular}

Table 1.3: Characteristics of selected fixed target experiments measuring high $p_{T}$ pion cross sections using a $\pi^{-}$beam.

of this topic is presented in [1]. Table 1.3 lists several fixed target experiments performed at Fermilab (E705, E111) and CERN (WA70, NA24, NA3) which measured high $p_{T}$ production of $\pi^{0}$ mesons by $\pi^{-}$beams as well as one (E258) which measured the production of charged pions.

E111 [26] was the first experiment to measure the $\pi^{0}$ production cross section by a pion beam. It used $\pi^{+}, \pi^{-}$and $\mathrm{p}$ beams at 100 and $200 \mathrm{GeV} / \mathrm{c}$ and a liquid hydrogen target. Measurements were made in the central rapidity region. The photon calorimeter, a lead-scintillator sandwich, served as the triggering device.

E258 [27] measured the production of charged hadrons by $\pi^{-}$beams at 200 and $300 \mathrm{GeV} / \mathrm{c}$ on a variety of targets. A magnetic spectrometer was used to observe particles in the central rapidity region. 
Other experiments, E705 [28], NA3 [29], NA24 [30] and WA70 [31] were dedicated direct photon experiments. They also measured $\pi^{0}$ and $\eta$ cross sections over a wide rapidity range using hydrogen or nuclear targets. Each of them featured electromagnetic calorimetry to detect and trigger on high $p_{T}$ showers.

From Table 1.1 it can be seen that E706 measured $\pi^{0}$ production on a variety of targets and over a wide range of rapidities. The high statistics of these data allow for a precise determination of the cross sections as functions of $p_{T}$ and rapidity as well as measurements of nuclear dependence. Comparisons with the NLL theoretical predictions can also be made. Since the meson production cross sections behave as $\sigma \sim p_{T}^{-n}$, where $n \sim 9.5$ (see Chapter 6), this test of pQCD spans many orders of magnitude of the $\pi^{0}$ inclusive cross section. 


\section{Chapter 2}

\section{The E706 Spectrometer}

E706 was located in the Meson West (MWEST) experimental hall of Fermi National Accelerator Laboratory. It took data concurrently with di-muon experiment E672. The layout of both experiments is illustrated in Figure 2.1. The laboratory coordinate system had its $\mathrm{Z}$ axis pointing in the direction of the beam (north), the $\mathrm{Y}$ coordinate pointing up and $\mathrm{X}$ pointing west, with the origin near the experimental target.

The E706 spectrometer consisted of two major sections:

- a charged particle tracking system consisting of a series of Silicon Strip Detectors (SSDs), a dipole analysis magnet, Proportional Wire Chambers (PWCs) and Straw Drift Tubes (STRAWs) chambers.

- calorimetry provided by a Liquid Argon Calorimeter (LAC) divided into Electromagnetic (EMLAC) and Hadronic (HALAC) sections and a Forward Calorimeter (FCAL). The main E706 trigger was a high $p_{T}$ shower in the EMLAC.

The E672 apparatus located downstream from E706 consisted of a toroidal analysis magnet, sets of muon PWCs and muon trigger hodoscopes. 


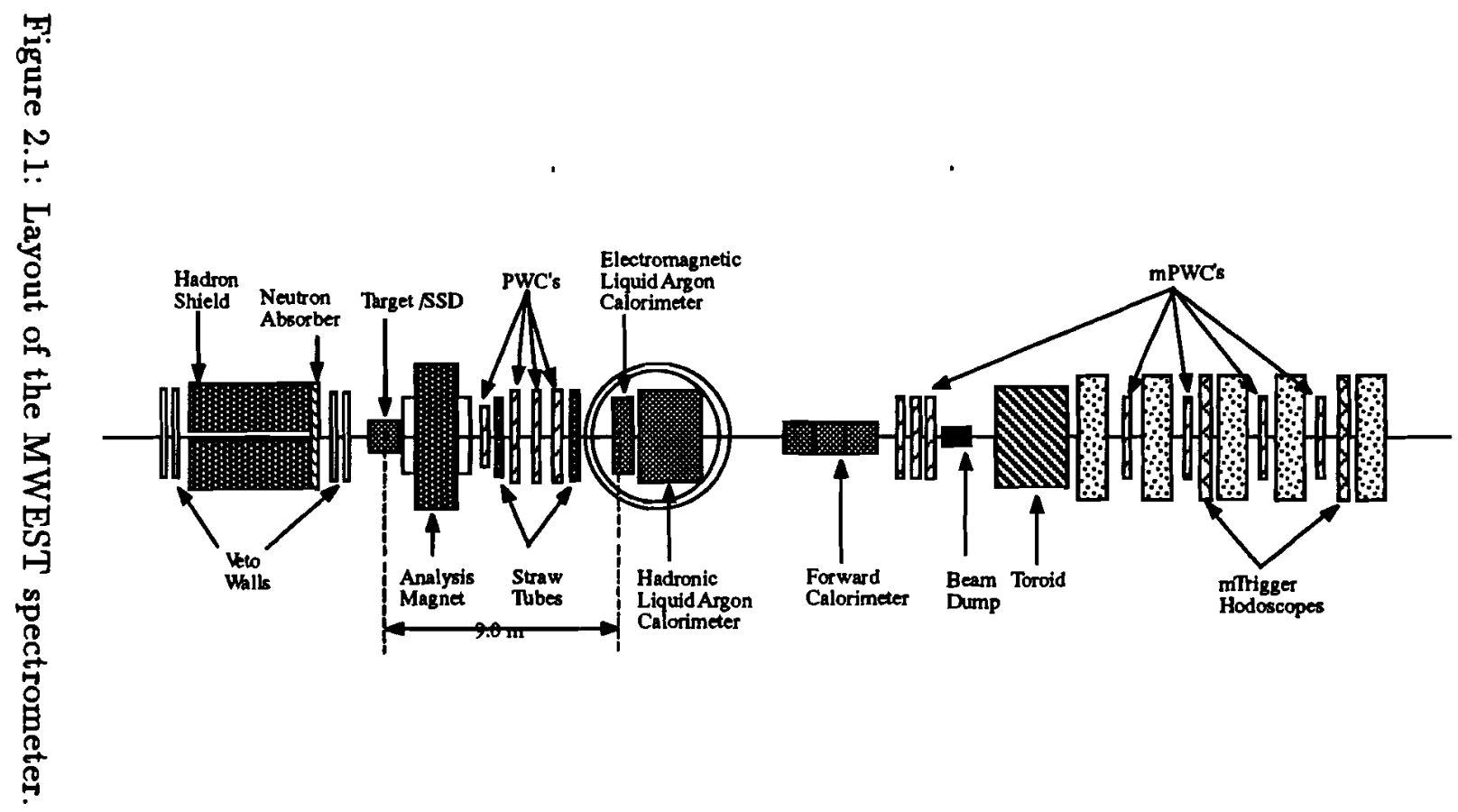

1

1

1

1




\subsection{The Meson West Beamline}

The Meson West (MW) beamline was capable of transporting beams of secondary particles in the momentum range from 25 to $1000 \mathrm{GeV} / \mathrm{c}$ as well as the $800 \mathrm{GeV} / \mathrm{c}$ primary proton beam (Figure 2.2). The beamline was split into two parts: the primary, which transported protons to the production target and selected polarity, and the secondary, which delivered a focused beam to the experiment target. The primary section of the MW beamline comprised enclosures MW1 to MW6, the secondary MW7 to MW8.

After being extracted from the Tevatron ring, the $800 \mathrm{GeV} / \mathrm{c}$ primary proton beam was split in the Switchyard among three experimental areas, Meson, Neutrino and Proton. Each portion was then distributed among the several experiments operating in those areas. The $\mathrm{MW}^{1} \mathrm{~W}^{1}$ magnet split the Meson beam among the MW, MC and MP beam lines. The amount of the beam delivered to the MW beamline depended on the mode in which E706 was operating. In the primary beam mode only about 1 percent of the Tevatron protons was directed down the MW primary beamline, while in the secondary beam mode, in which $800 \mathrm{GeV} / \mathrm{c}$ protons were used to produce pion beams, as much as $30 \%$ of the total Tevatron beam ( $\sim 5 \times 10^{12}$ protons per 23 seconds spill) was delivered to the MWEST production target. The particle composition of the secondary $\pi^{-}$beam was: $97 \%$ pions, $2.9 \%$ kaons and $0.1 \%$ antiprotons [32].

Upon entering MW2, the beam was first bent and then focused on the position of the secondary beam production target, a 1.14 interaction length block of aluminum. Dipole magnet MW6W was used as a simple bend when running in the primary beam mode, and as the secondary beam polarity and energy selector when

\footnotetext{
${ }^{1}$ The coded names of the magnets contain information about their type and location. For example, MW7Q1 describes the first quadrupole (Q1) located in Meson West enclosure number 7 (MW7). MW2W is a dipole bending to the west (W) located in enclosure number 2 (MW2).
} 


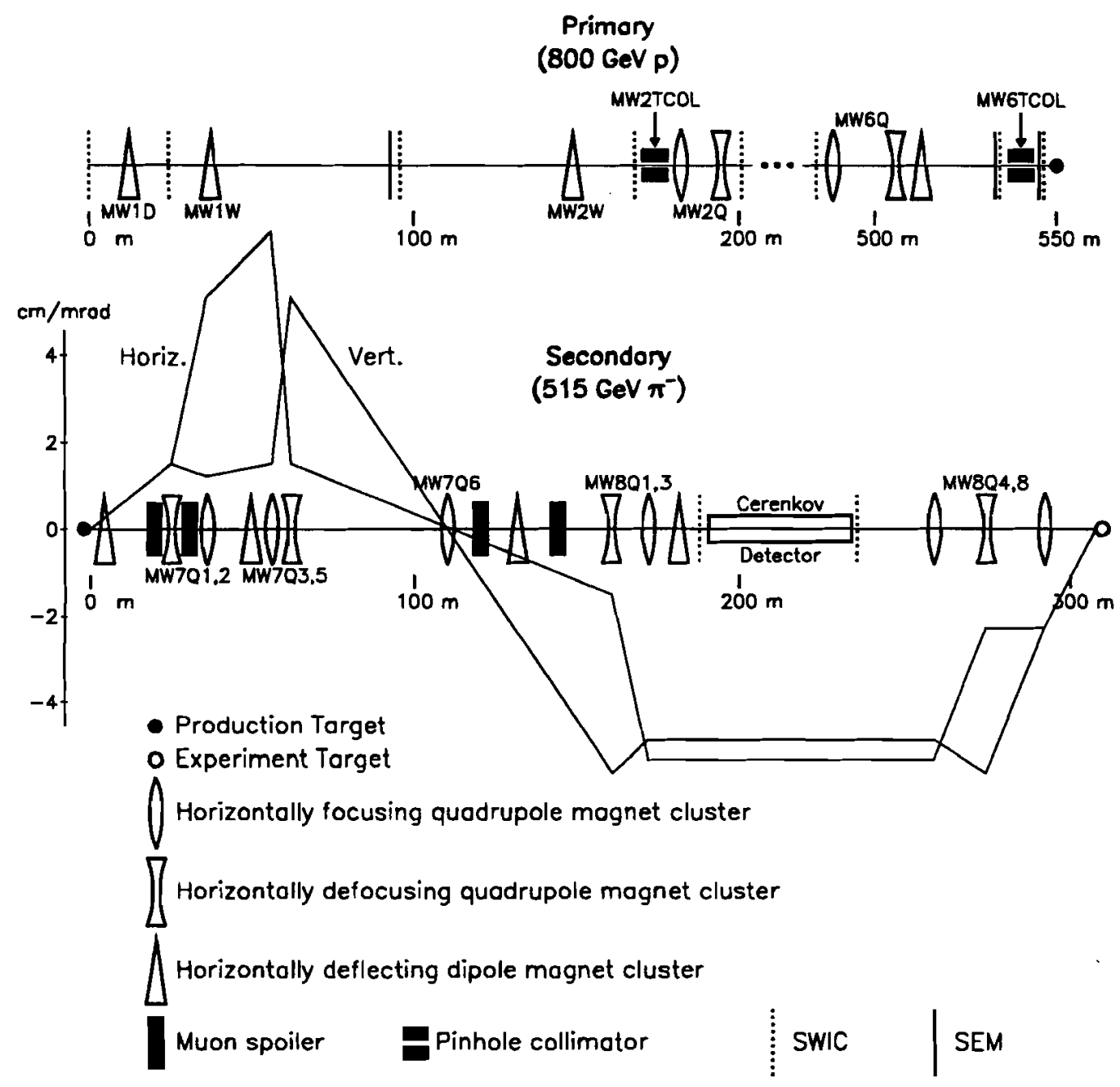

Figure 2.2: The Meson West beamline. 
running in that mode ${ }^{2}$. Other elements of the beamline, which did not actively participate in the beam transfer process were:

- Segmented Wire Ion Chambers (SWICs), used to monitor the $x$ and $y$ profiles and positions of the beam.

- Secondary Emission Monitors (SEMs), used to measure the beam intensity.

- Two long pinhole collimators (apertures of 1 and $2 \mathrm{~mm}$ ), used only in the primary beam mode to attenuate the proton beam by a factor of $1 / 2000$, as required by the radiation safety rules. Their alignment was a time consuming task, as step motors allowed for the independent movement of both ends of each collimator.

The lower part of Figure 2.2 shows the basic components of the secondary beamline. The sinelike trajectories of the beamline transfer matrix as determined by TRANSPORT program [32] are also shown. The main focusing components were:

- asymmetric quadrupole triplet MW7Q1-2, MW7Q3-4, MW7Q5. It produced a point image of the primary target at the location of MW7Q6.

- field lens MW7Q6. Its function was to remove momentum dispersion of the beam, as caused by bending magnets (for example MW6W), so the beam could be focused at the experiment target.

- quadrupole doublet MW8Q1-2, MW8Q3. It produced a parallel section of the beam for the Cerenkov counter.

- quadrupole triplet MW8Q4, MW8Q5-6, MW8Q7-8. It refocused the beam at the experiment target.

\footnotetext{
${ }^{2}$ In Figure 2.2, MW6W is the first magnet following the experiment target.
} 
Other (nonfocusing) elements of the secondary beamline were:

- bending magnets required due to the curvature of the beam tunnel.

- spoiler magnets for sweeping away the particles (mainly muons) travelling parallel to the beam outside the beam pipe.

- variable aperture collimators to control the size of the beam profile.

- SWIC and SEM monitors.

- a differential Cerenkov counter located $90 \mathrm{~m}$ upstream from the experiment target. It used helium gas at a pressure of $\sim 6$ psia and was able to tag three components of the secondary beam simultaneously.

The method used to determine the beam momentum will be described in Chapter 5 .

\subsection{The Target Region}

A $4.7 \mathrm{~m}$ long steel hadron shield was placed upstream of the target in order to absorb beam halo particles which were not swept out by the spoiler magnets. In addition, three large walls of scintillation counters (veto walls) were located on the ends of the shield ${ }^{3}$. Their signals were used in the on-line definition of the trigger (Chapter 3) and during the off-line analysis (Chapter 5). Neutrons produced in the shield were absorbed in a water tank placed at the downstream end of the shield.

A set of scintillation counters was used to detect the presence of an incident particle in the beam buckets. This set consisted of three planes of overlapping scintillation paddles, referred to as the beam hodoscope, and one plane with a 1

\footnotetext{
${ }^{3} \mathrm{~A}$ fourth veto wall was added during the 1991 run.
} 
$\mathrm{cm}$ hole, referred to as the beam hole (BH) counter. These counters were centered on the SSD chambers. The beam hodoscope paddle widths ranged from $1 \mathrm{~mm}$ in the central region to $5 \mathrm{~mm}$ on the edges. They covered an area of $2 \times 2 \mathrm{~cm}^{2}$. A set of scintillation counters, two upstream of the analysis magnet (SE1, SW1) and another two downstream (SE2, SW2), all centered on the beamline were used to identify interactions in the target. Their sizes were $7.6 \times 15.2 \mathrm{~cm}^{2}$ and $10.2 \times 20.4$ $\mathrm{cm}^{2}$ respectively. Interactions of the beam in the target produced signals in one or more counters. Hodoscope, beam hole and interaction counters information was latched for each beam bucket and the status of these counters was written to tape for 7 buckets preceding and 7 following the one for which a trigger was accepted.

During the 1990 run, two nuclear targets, copper and beryllium, were used in order to study the nuclear dependence of the production of mesons, photons and jets (see Figure 2.3); during the 1991 run, a liquid hydrogen target was added. The target material was located approximately $1.5 \mathrm{~m}$ downstream of the hadron shield. Two $0.8 \mathrm{~mm}$ thick copper pieces were followed by two $1 \mathrm{~cm}$ diameter beryllium cylinders. Their lengths were 3.7 and $1.1 \mathrm{~cm}$. Table 2.1 presents the target characteristics. In addition, a significant number of events had their primary vertices in SSD planes, so they were also effectively $\mathrm{Si}$ targets.

\subsection{The Tracking System}

\subsubsection{The Silicon Strip Detectors}

E706 used eight pairs of SSD chambers as the upstream component of the tracking system [33], Figure 2.3. Three of them, located upstream of the experiment target, served as beam chambers, the remaining five pairs placed downstream of the target were used to determine the locations of the event vertices (primary and secondary), and in conjunction with PWCs and STRAWs to reconstruct charged 


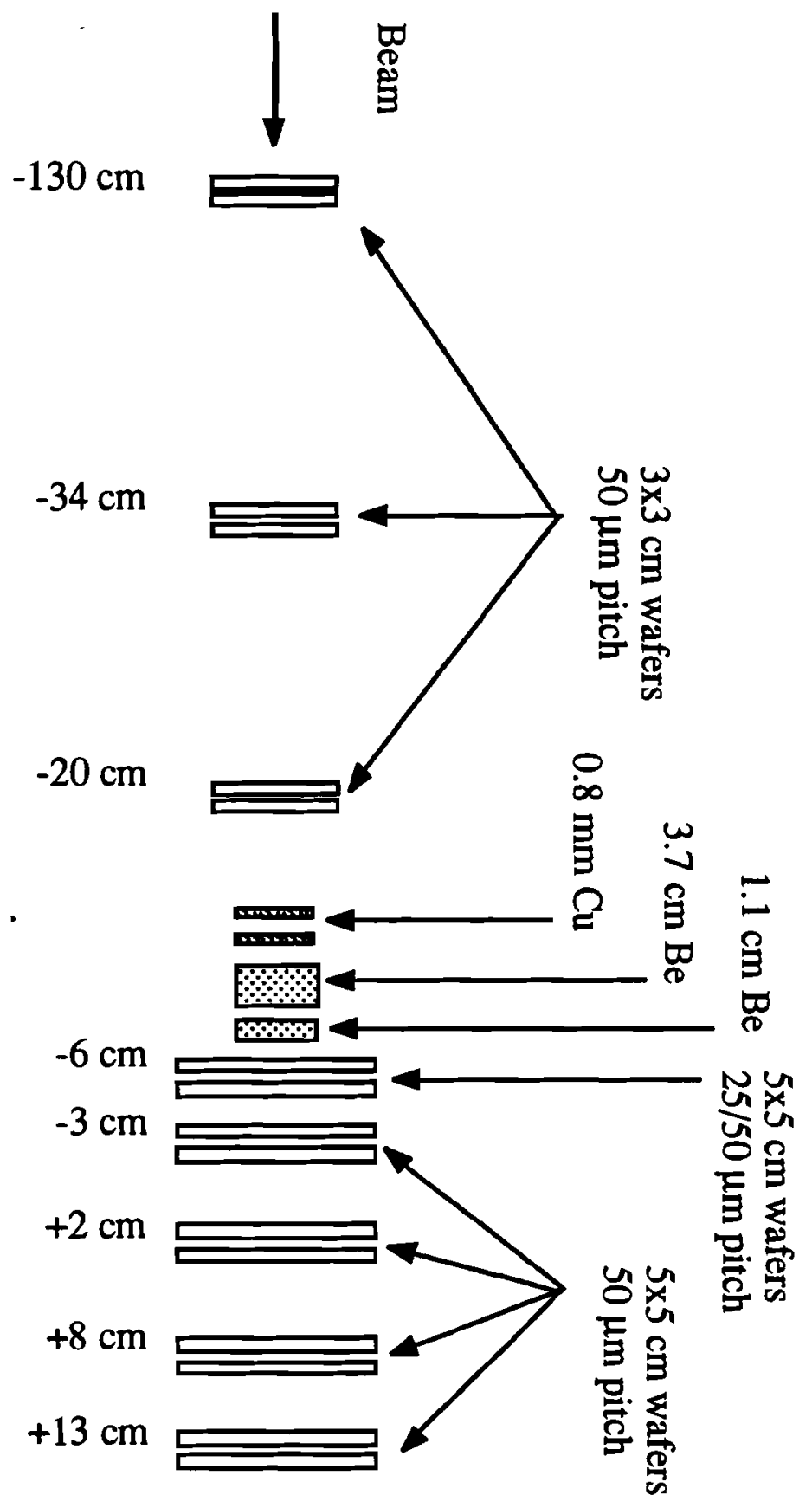

Figure 2.3: The 1990 E706 target region setup. 


\begin{tabular}{|c|c|c|c|}
\hline \hline Material & $\begin{array}{c}\text { z Position } \\
(\mathrm{cm})\end{array}$ & $\begin{array}{c}\text { Radius } \\
(\mathrm{cm})\end{array}$ & $\begin{array}{c}\text { Interaction } \\
\text { Lengths (\%) }\end{array}$ \\
\hline \hline $\mathrm{Cu}$ & -15.52 & 1.27 & 0.8 \\
-15.19 & 1.27 & \\
$\mathrm{Be}$ & -12.79 & 1.0 & \\
\hline
\end{tabular}

Table 2.1: The E706 targets.

particle trajectories.

Each SSD pair consisted of a silicon wafer (approximately $270 \mu \mathrm{m}$ thick) with multiple metallic anodes in the form of thin strips parallel to the $\mathrm{X}$ axis in the lab coordinate system, followed by a wafer with anodes parallel to the $Y$ axis. Each of the anodes acted as an independent detector and had its own readout circuitry. The separation between the anodes (pitch) determined the resolution of the SSD chambers. Locations and sizes of the instrumented regions of the SSD pairs and their pitch values are listed in Table 2.2.

\subsubsection{The Analysis Magnet}

The dipole analysis magnet was located downstream of the vertex SSD chambers. The $\mathrm{Z}$ coordinate of its center was $2 \mathrm{~m}$. During the run it operated at a current of $1050 \mathrm{~A}$ producing a magnetic field of $6.2 \mathrm{kG}$ which delivered a $450 \mathrm{MeV} / \mathrm{c}$ momentum impulse in the $\mathrm{X}$ direction. The magnet aperture was filled with helium to minimize the effects of multiple Coulomb scattering.

The uniformity of the dipole field was improved by installing mirror plates on 


\begin{tabular}{|c|c|c|}
\hline \hline $\begin{array}{c}\text { z Position } \\
(\mathrm{cm})\end{array}$ & $\begin{array}{c}\text { Wafer Size } \\
\left(\mathrm{cm}^{2}\right)\end{array}$ & $\begin{array}{c}\text { Pitch } \\
(\mu \mathrm{m})\end{array}$ \\
\hline \hline-130 & & 50 \\
-34 & $3 \times 3$ & 50 \\
-19 & & 50 \\
& & \\
\hline & & $25 / 50$ \\
-6 & & 50 \\
+2 & $5 \times 5$ & 50 \\
+8 & & 50 \\
+13 & & 50 \\
\hline \hline
\end{tabular}

Table 2.2: Parameters of the E706 SSD chambers.

both ends of the magnet. These mirror plates significantly reduced the intensity of the fringe field at the locations of SSDs and PWCs, and the remaining fringe field effects were taken into account in the field parametrization.

\subsubsection{The Proportional Wire Chambers and Straw Drift Tubes}

The downstream section of the tracking system consisted of four modules of PWCs [34] and two modules of STRAWs [35]. They were located between the analysis magnet and the Liquid Argon Calorimeter. The active area of the chambers varied from $1.22 \times 1.22 \mathrm{~m}^{2}$ to $2.44 \times 2.44 \mathrm{~m}^{2}$. Each PWC module contained 4 anode planes (so called $Y, U, X$ and $V$ view planes) with wires making $0,37,90$ and -53 degree angle with the $\mathrm{Y}$ axis in the laboratory coordinate system. Each of the anode planes was placed between two cathode planes. The anode - cathode separation was $5.74 \mathrm{~mm}$. Cathodes were made out of graphite coated $25 \mu \mathrm{m}$ thick mylar sheets. The central (beam) areas of the PWCs were desensitized by causing the 
HV to drop as the current in that region increased. The anode wires (gold plated tungsten) were 0.8 mil (approximately $20 \mu \mathrm{m}$ ) in diameter and were separated by $2.5 \mathrm{~mm}$. The PWC gas composition was $80 \%$ argon, $18 \%$ isobutane and a small amount of freon and isopropyl alcohol.

The first of the two STRAW chambers was located upstream of the PWCs and the second one was placed in front of the LAC. Each chamber consisted of four $\mathrm{X}$ and four $\mathrm{Y}$ view planes of aluminized mylar tubes. The diameter of the tubes was $10.4 \mathrm{~mm}$ in the first and $15 \mathrm{~mm}$ in the second chamber. The anodes were made of $20 \mu \mathrm{m}$ gold plated tungsten. The gas mixture consisted of $50 \%$ argon and $50 \%$ ethane. The central regions of the STRAWs were desensitized.

The tracking system had approximately 25000 instrumented channels. PWC and SSD readout was performed by a CAMAC based system. STRAWs readout was integrated with the LAC readout system.

\subsection{Calorimetry}

\subsubsection{The Liquid Argon Calorimeter}

The Liquid Argon Calorimeter [36, 37], located $9 \mathrm{~m}$ downstream from the target, was the central component of the E706 spectrometer. It was used to measure the energies and positions of the photons produced in the interactions. It was also the main component of the trigger system.

The LAC was suspended from a mobile gantry and enclosed in the steel cryostat as illustrated in Figure 2.4.

The dewar vessel (bottom part of the cryostat) was bolted to the cap attached to the gantry. The stainless steel wall of the vessel was $1.6 \mathrm{~cm}$ thick and was insulated by $25 \mathrm{~cm}$ of fiberglass and polyurethane foam. An opening, $10 \mathrm{~cm}$ in diameter, in the center of the wall provided a window for the beam particles. Because of the 


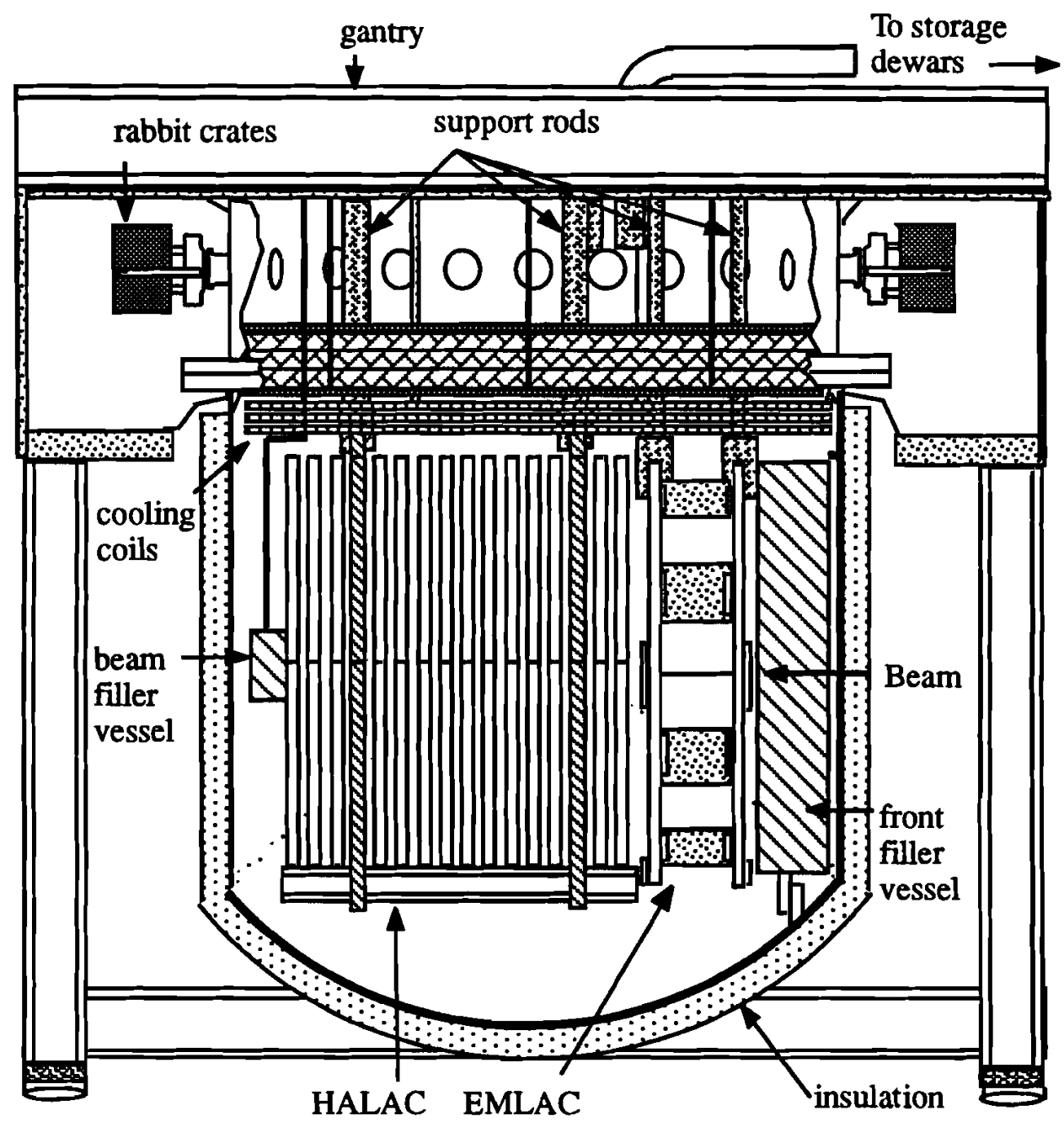

Figure 2.4: A side view of the LAC. 
cylindrical geometry of the dewar, there was a large volume between its wall and the flat face of the calorimeter. A front filler vessel, made out of $1.6 \mathrm{~mm}$ steel and filled with low density foam, was inserted in that region to displace argon in order to minimize the amount of material in front of the LAC. Argon was also displaced from the beam region of the dewar by a $40 \mathrm{~cm}$ diameter pipe filled with helium.

The cap (upper part of the cryostat) not only provided the cover for the dewar but also housed the cryogenic piping, LAC High Voltage system and part of the calorimeter readout and trigger electronics. A number of portholes installed around the cap made connection between the calorimeter and the outside world possible; they housed feedthroughs for the signal and HV cabling. Because the amplification of the calorimeter signals was done outside the dewar, the area around the cap was enclosed with galvanized sheet metal and was referred to as the 'Faraday room'. All power and signal cables that were fed through the 'Faraday room' wall used transformers or optical couplers. Once assembled, the whole calorimeter structure could be moved on a set of Hillman rollers along the $\mathrm{X}$ axis. This transverse motion (coupled with the effect of a vertical dipole in the secondary beamline) made it possible to sweep a calibration beam over the entire face of the LAC. (The vertical cut in the hadron shield allowed only for a vertical deflection of the beam.)

The desire to detect high $p_{T}$ electromagnetic showers caused by direct photons or decay products of the particles created in the vertex, put the following constraints on the design of the LAC:

- The calorimeter had to be able to distinguish between photons (electrons) and hadrons. For this purpose, the LAC was divided into two sections: the EMLAC characterized by large radiation and small interaction lengths, and the HALAC able to contain the majority of the hadronic showers.

- The detector had to be capable of triggering on events of interest. 
- The EMLAC had to be deep enough to contain electromagnetic showers and the thickness of the sampling cell had to be optimized for a fast risetime of the signal (narrow argon cells) while maintaining reasonable signal to noise ratio (wide argon cells).

- The lateral segmentation of the EMLAC had to be fine enough to resolve photons from the decays of high energy neutral pions ${ }^{4}$ so that the background to the direct photon sample would be determined accurately.

\section{The EMLAC}

The EMLAC, illustrated in Figure 2.5, was a sampling calorimeter with lead plates as absorbers and liquid argon as the ionization medium. It had an annular shape with an inner radius of $20 \mathrm{~cm}$ and an outer radius of $165 \mathrm{~cm}$. The EMLAC was divided into four independent quadrants. Each quadrant consisted of 33 sampling cells with strip type readout in polar $(r-\phi)$ geometry. The EMLAC total thickness was $75 \mathrm{~cm}$. Each cell consisted of four electrodes with $2.5 \mathrm{~mm}$ gaps of liquid argon between them. The first electrode in the cell, a $2 \mathrm{~mm}$ thick lead cathode ${ }^{5}$ was kept at $2.5 \mathrm{kV}$ and had two $100 \mathrm{nf}$ ballast capacitors attached to it. Next came an $r$ type (view) anode board followed by a second lead cathode and a $\phi$ type (view) anode board. Each anode board was made from double sided copper clad $1.59 \mathrm{~mm}$ thick G-10, and each side (a quadrant) was electrically separated into two octants, called left and right. The EMLAC was approximately 30 radiation lengths thick, with lead accounting for $86 \%$ of the radiation lengths. The readout strips were milled on both sides of the anode boards, forming a tower structure focused on a point $9 \mathrm{~m}$ upstream from the front face of the EMLAC. The layout of the first

\footnotetext{
${ }^{4}$ The separation between two photons detected in the EMLAC and produced in $\pi^{0}$ decay is approximately $\left(240 \mathrm{GeV} / E_{\pi^{0}}\right) \mathrm{cm}$ for $\Theta^{*}=90^{\circ}$. For example, photons from a $100 \mathrm{GeV}$ pion are separated by $2.4 \mathrm{~cm}$.

${ }^{5}$ In the first cell this cathode was made of $\mathrm{Al}$.
} 


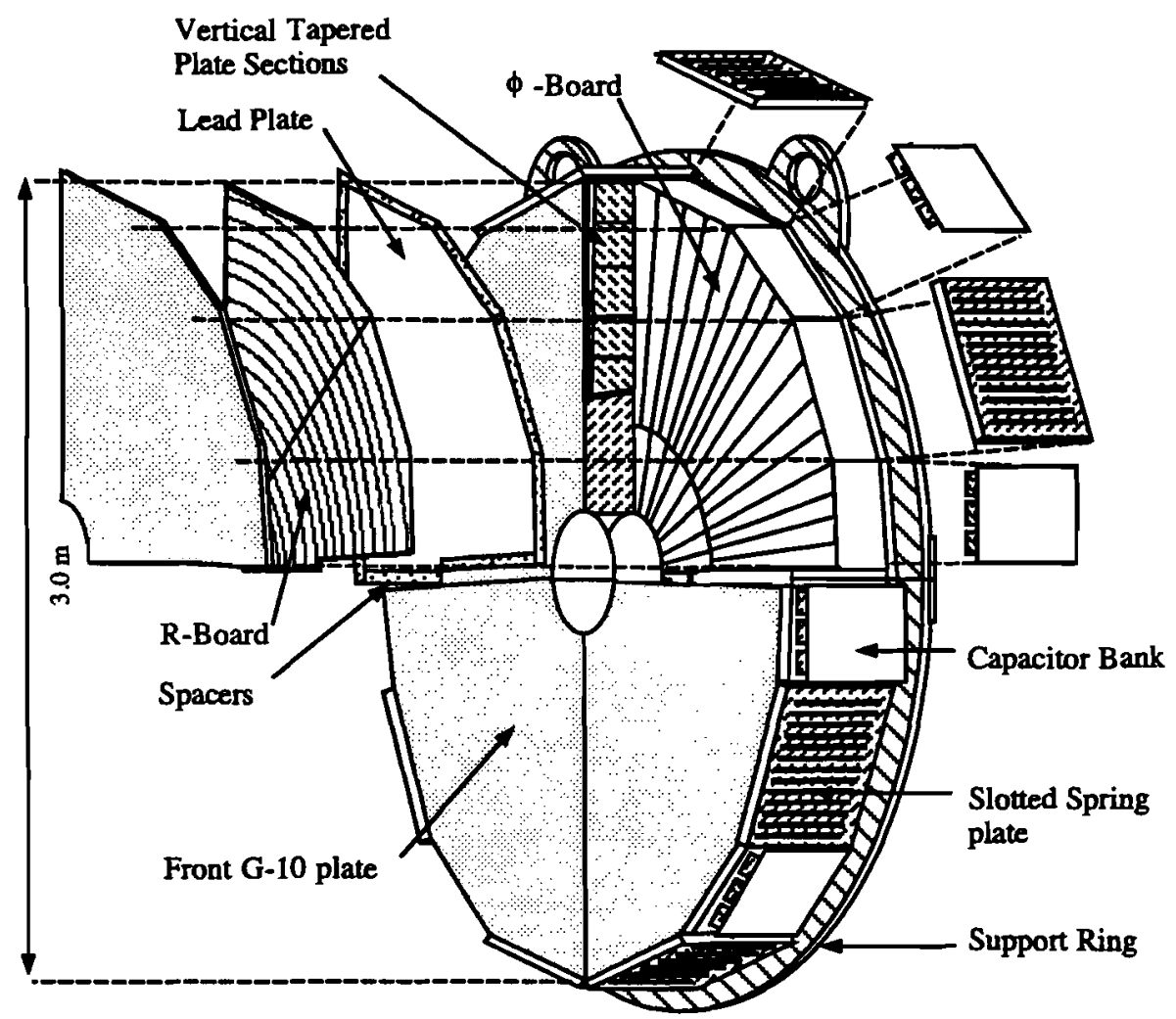

Figure 2.5: Exploded view of the EMLAC. 
$r$ board determined the geometry of the remainder and caused some peculiarities in the tower structure. The first $r$ board was divided into 254 readout strips; 252 of them were of the same width equal to $0.5456 \mathrm{~cm}$, while the 2 boundary strips (called inner and outer flash) were wider. The tower geometry caused the inner flash strip on the following boards to be wider. On the board belonging to the 15th cell it was wide enough to accommodate an additional readout strip which did not have partners on the upstream boards. The last five $r$ boards had two such strips. The opposite happened on the outer edge of the detector. The last $r$ board had 16 outer strips missing, because of the tower structure of the EMLAC. The numbering convention for $r$ strips was as follows: inner flash was number 0 , while the number of the outer flash strip was 255 .

The $\phi$ boards were divided into two parts: inner and outer $\phi$. The inner-outer boundary was at $40.2 \mathrm{~cm}$, which corresponded to the outer edge of the strip number 36 on the first $r$ board. One inner $\phi$ strip subtended 1/96 of a right angle. For the outer strips the fraction was $1 / 192$. In an ideal situation this would translate to 48 inner and 96 outer strips per octant. In reality, some of the strips on the quadrant boundaries did not exist in order to accommodate the calorimeter support structure. This produced gaps in the azimuthal acceptance of the EMLAC.

Charge collected by the anode strips was read out using special 'readout boards' placed on both ends of the EMLAC. The upstream board read out the first 11 cells (front section), while the downstream one read out the remaining 22 (back section). The signal in an output channel was formed by ganging together strips that subtended the same portion of the solid angle and belonged to the same section. The first and the second $r$ strips were summed only in the back section, while strips 239 to 254 were summed only in the front. 


\section{The HALAC}

As mentioned before, the division of the EMLAC into front and back sections helped to distinguish electromagnetic showers from hadronic ones. The majority of the electromagnetic shower energy should be deposited in the front section of the EMLAC and the whole shower should be contained within the EMLAC volume. This could not be said about hadrons. The total interaction length of the EMLAC was too small for most hadronic showers to fully develop. The HALAC [38], another sampling calorimeter, was built to measure the energies and position of strongly interacting particles. The HALAC depth was 8 interaction lengths and it used stainless steel as an absorber. There were $531.0 \mathrm{~cm}$ thick sampling cells, referred to as 'cookies' (see Figure 2.6), separated by $2.54 \mathrm{~cm}$ thick steel plates.There were 14 cookies in the front section and 39 in the back.

The first element of a cookie was an $0.8 \mathrm{~mm}$ thick copper clad G-10 board. The upstream copper layer was grounded and the downstream was maintained at high voltage. $3 \mathrm{~mm}$ thick G-10 strips glued to the boards provided gaps for the liquid argon. The next element, a readout board, had triangular readout pads facing the HV plane. Charge collected on the pads was read out on the outer edge of the detector through the set of strips milled between rows of triangles. The space between the readout strips and corresponding areas on the BV plate was filled with $\mathrm{G}-10$, to make sure that no charge would be picked up by the readout strips. The second half of the cookie was a mirror image of the first, shifted upwards by one row of pads, so the space filled with G-10 on the previous board was an active argon gap. The triangular pads were focused on the target and those covering the same element of the solid angle were read out as a single channel in the front or in the back section. The size of the pads increased from $10 \mathrm{~cm}$ on the upstream edge to $14 \mathrm{~cm}$ on the downstream, and about $90 \%$ of the hadronic shower was contained 


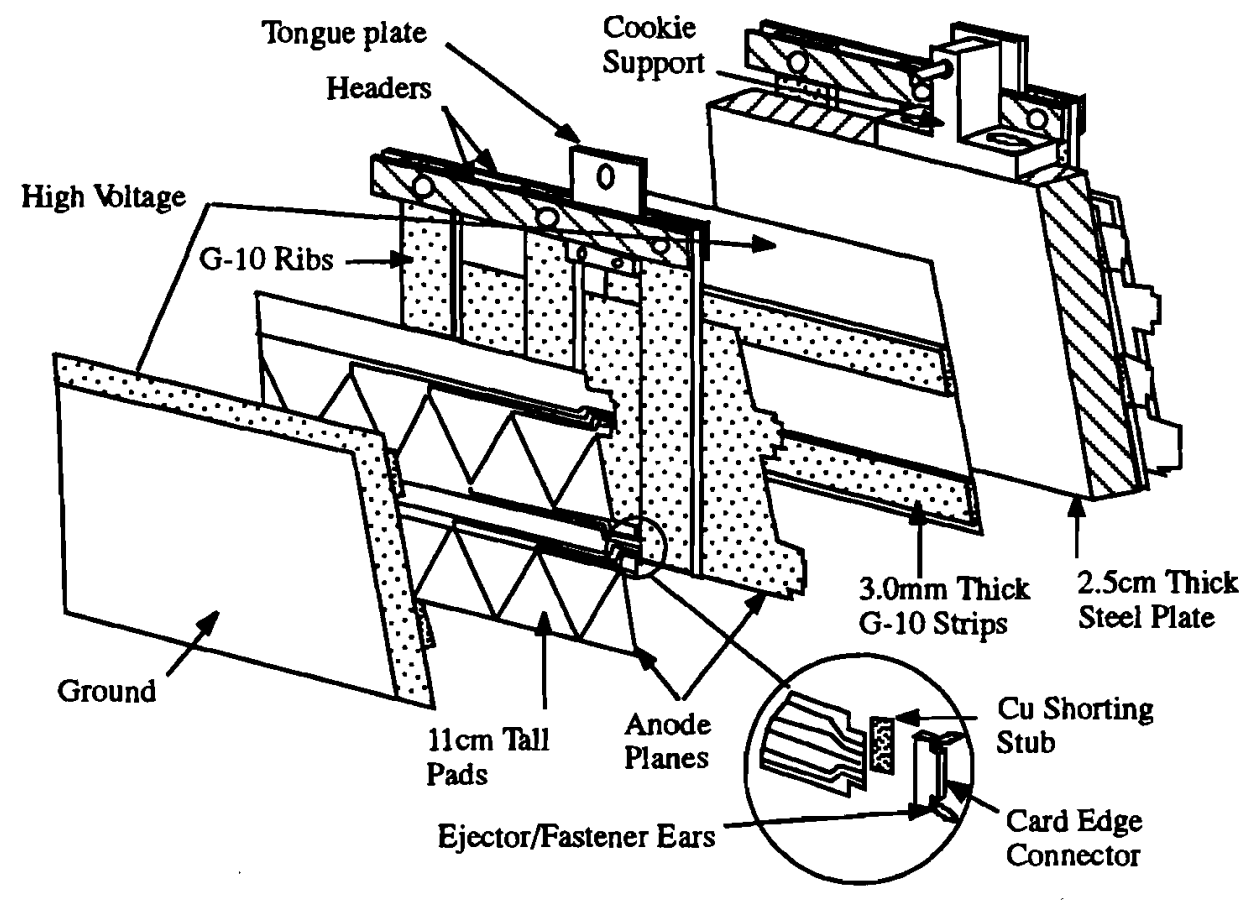

Figure 2.6: Structure of the HALAC cell. 


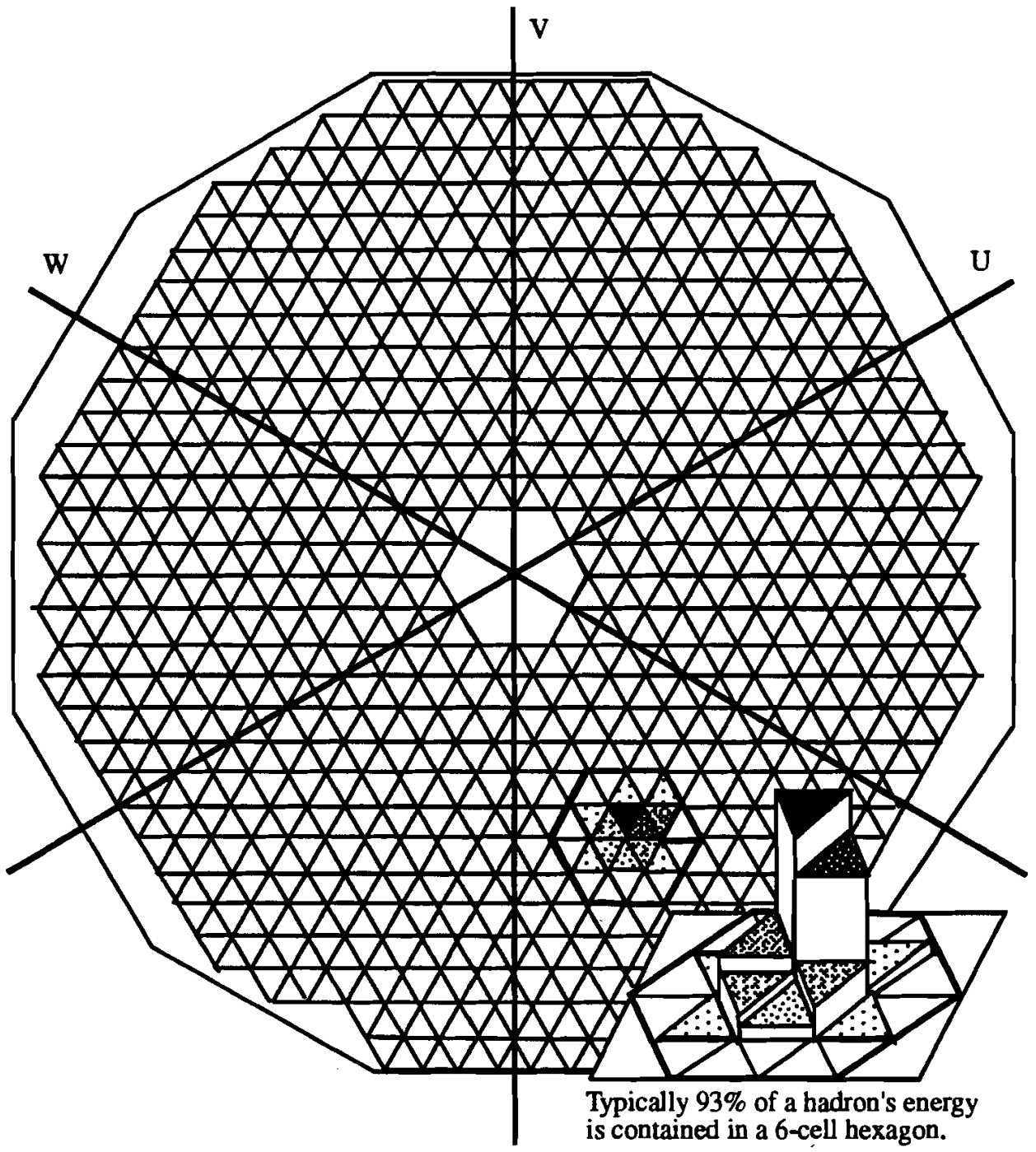

Figure 2.7: Geometry of the HALAC readout pads. 
in six neighboring elements (see Figure 2.7).

The EMLAC and the HALAC used the same electronic readout system. Signals from the readout boards were sent through the 28 portholes in the cap of the dewar to the Faraday room. Each of the ports accommodated 320 LAC channels. There were 8640 instrumented LAC channels, 6272 of the EMLAC and 2368 of the HALAC. For each event, the digitized results from every channel were written to the tape. The LAC data acquisition (DA) system will be described in greater detail in Chapter 3.

\subsubsection{The Forward Calorimeter}

The Forward Calorimeter (FCAL) [39] was located $15 \mathrm{~m}$ downstream from the target. It was $114 \mathrm{~cm}$ in diameter and its geometrical acceptance covered the region of the beam hole in the center of LAC, except for a $1.5 \mathrm{~cm}$ hole in the center of the FCAL that provided a passage for noninteracting beam particles. This allowed for the measurement of the energy and mean $p_{T}$ of the forward jet. The FCAL was a sampling calorimeter with $1.9 \mathrm{~cm}$ thick steel plates as absorbers and $0.46 \mathrm{~cm}$ acrylic scintillator plates as sampling medium. It was divided into three similar modules. Light produced by the scintillator was collected by $601.0 \mathrm{~cm}$ in diameter BBQ wave shifter rods. The signals were sent using phototubes to CAMAC modules. 


\section{Chapter 3}

\section{The E706 Trigger and Data Acquisition System}

In this chapter, I will describe the basic principle behind the E706 trigger and Data Acquisition (DA) systems. The emphasis will be on the LAC DA. As the cross section for the direct photon production is relatively small, the trigger must be able to handle high intensity beams efficiently. Also a speedy DA system is needed to collect the information from thousands of data channels while minimizing the dead time.

\subsection{Overview}

A block diagram of the E706 DA system is shown in Figure 3.1.

Its central unit was a DEC $\mu$ VAX computer running VAXONLINE, a FERMILAB on line data acquisition software package [40]. Other components, slave modules for the $\mu \mathrm{VAX}$, were: FASTBUS [41] based LAC and STRAWs readout systems and three PDP-11 minicomputers for readout of the remaining components of the spectrometer through serial and parallel CAMAC links. The PDP minicomputers were known as: NEU - responsible for the readout of the trigger system, the PWCs, the SSDs and the scalers, ROCH - responsible for the readout of the FCAL, and MU - responsible for the readout of the E672 di-muon system. 


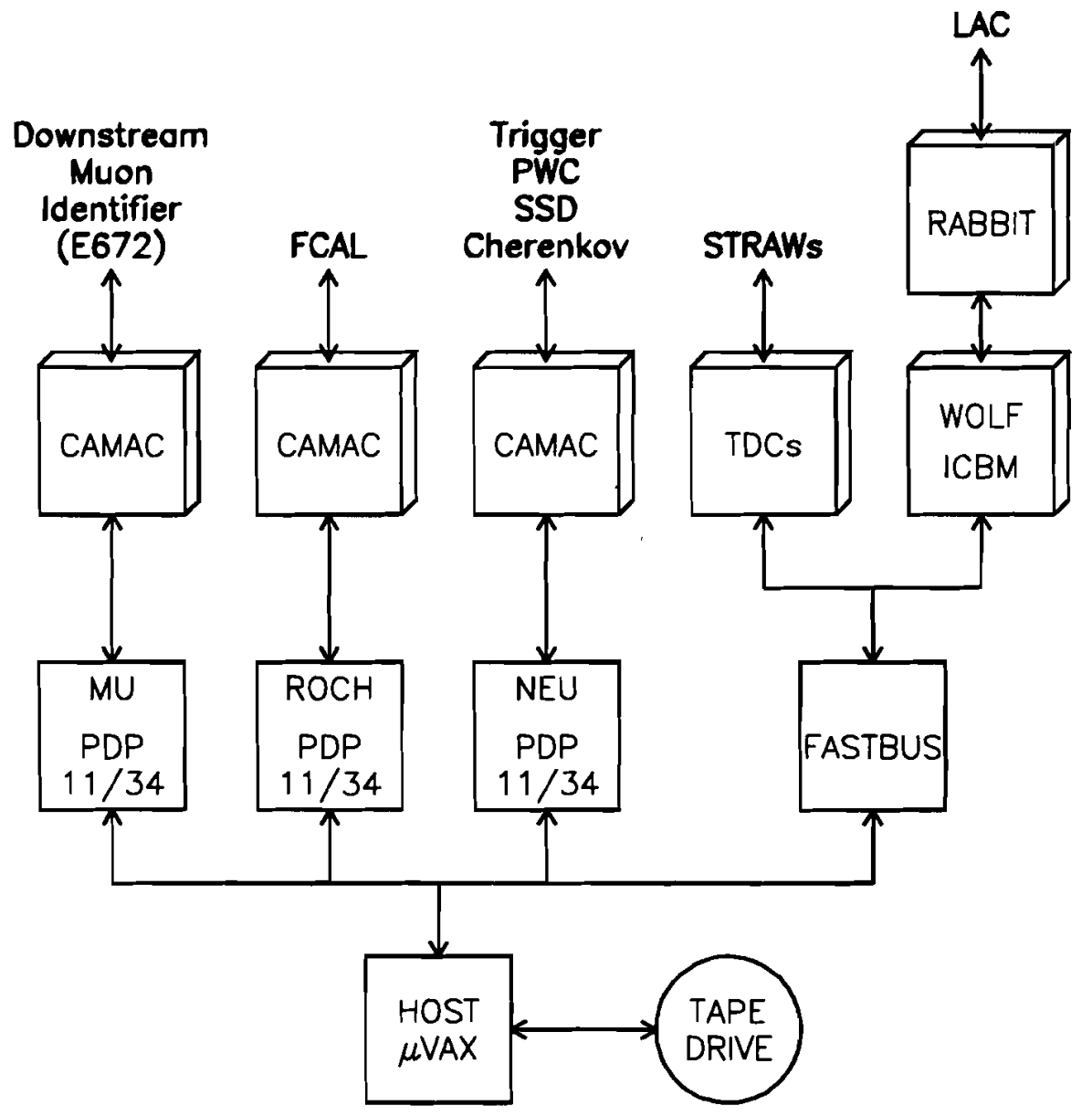

Figure 3.1: Block diagram of the E706 DA system. 
Data read out by the slave components (so called subevents), were concatenated by the $\mu \mathrm{VAX}$ and written to the $8 \mathrm{~mm}$ magnetic tape as one event. Some of the events were made available to another VAX for online monitoring. All these tasks were supervised by the following VAXONLINE processes:

- Global Menu - the user interface for all VAXONLINE processes.

- Run Control - the user interface to the DA system controlling the data taking process.

- Event Builder - it concatenated the data from the various subsystems into a complete event. Concatenation was based on matching the 'event number' in each subevent. This number was provided by one of the scalers called the Hydra Scaler.

- Output - it managed the writing of events to tape.

- Consumer Programs - analysed a fraction of the concatenated events and monitored the performance of scalers, latches, tracking system, LAC and DA system itself.

Events were written to tape in groups of so called runs. One run could contain a maximum of 65535 events. During normal running conditions, a new run was started every 2 hours. The readout system was reinitialized periodically (usually every eight hours) and at the same time the calibration data were taken for the detector components. We called this procedure a 'cold start'.

\subsection{The LAC DA System}

Figure 3.2 presents the part of the E706 DA system responsible for the LAC. The LAC DA was based on the FASTBUS standard. The electronic modules were 
housed in two FASTBUS crates called CRATE-11 and CRATE-20. CRATE-20 housed also elements of the STRAWs readout system.

\subsubsection{The RABBIT System}

As mentioned in Chapter 2, $r$ and $\phi$ strips of the EMLAC (pads in the case of HALAC) covering the same solid angle, were ganged together and treated as one electronic channel. The risetime of the signal (as dictated by the geometry of the LAC) was about $800 \mathrm{~ns}$. Since the LAC was the primary triggering element of the experiment, a sophisticated amplifier card (LACAMP) was designed by members of the E706 and FERMILAB Particle Instrumentation Group [42]. These cards were integrated into the RABBIT readout system that had been developed at FERMILAB [43].

RABBIT was a modular data handling system. Its basic unit was a crate that usually contained two controller (EWE) cards, 20 front end amplifier cards (LACAMPS in the case of E706) and a terminator card (BAT) as illustrated in Figure 3.3.

The backplane of the crate had two (largely redundant) analog buses called Top and Bottom. Each of them could be controlled by either of the EWEs. There were 28 RABBIT crates in the Faraday Room. They communicated with FASTBUS CRATE-11 via $200 \mathrm{ft}$ long cables.

\section{The LACAMP Card}

A diagram of a LACAMP card is presented in Figure 3.4.

Each card could handle up to 16 input LAC channels. Figure 3.4 shows 4 of them in greater detail. The signal from one LAC channel, whose strength varied from 30 to $250 \mathrm{fC} / \mathrm{GeV}$, was first amplified by a low impedance integrating amplifier. The resulting signal was then fanned out to three different circuits: 


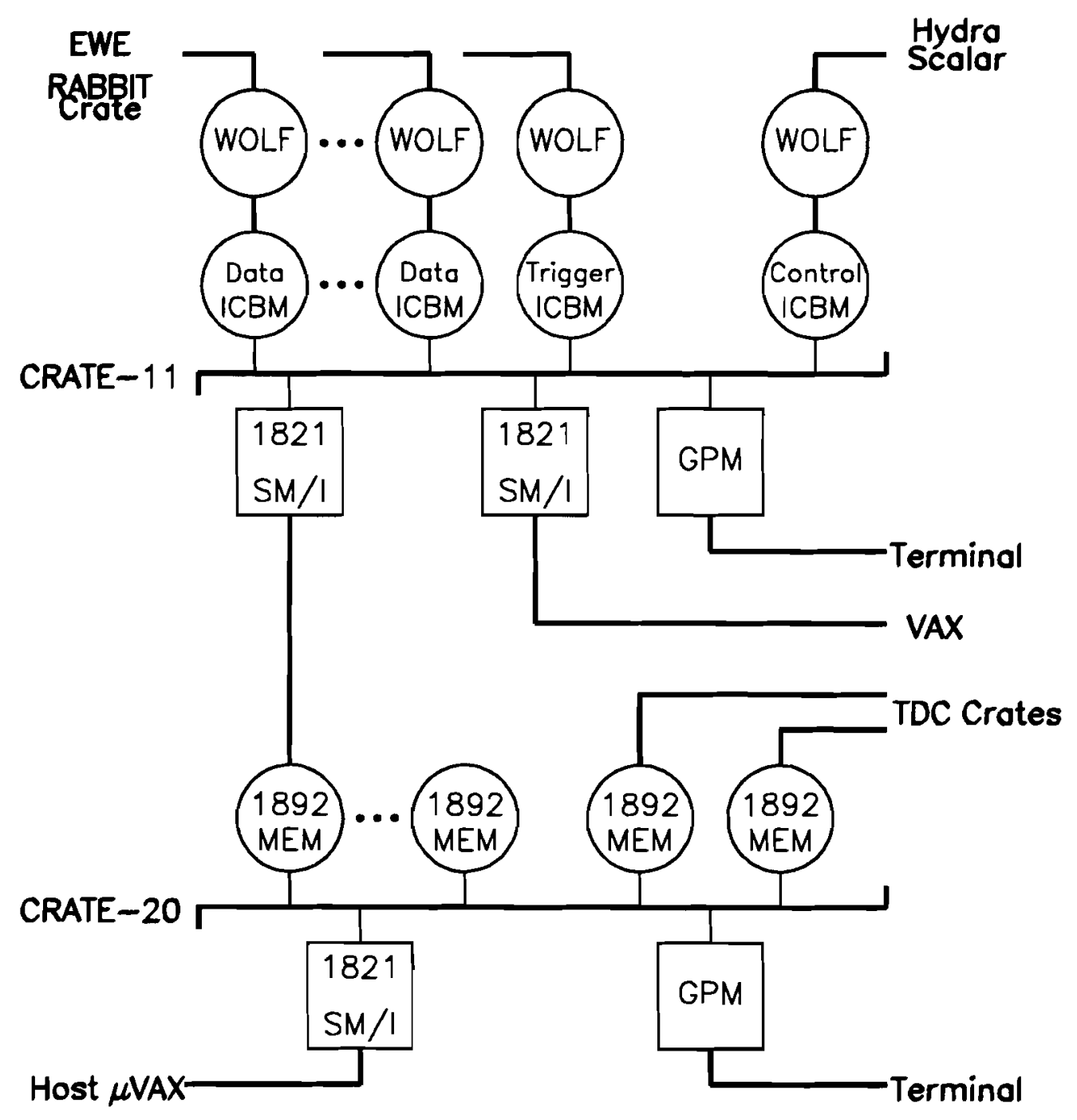

Figure 3.2: The LAC DA system. 


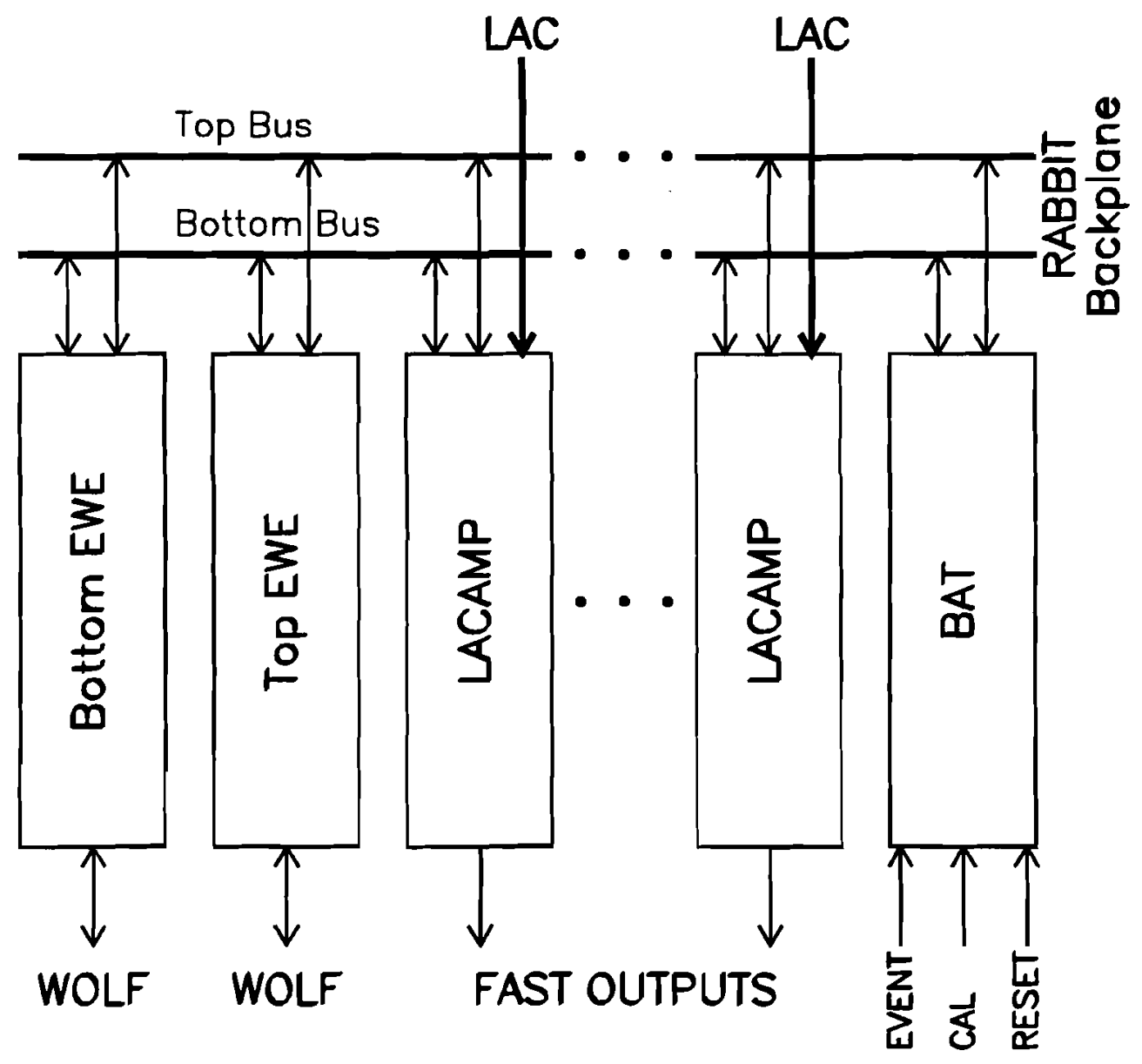

Figure 3.3: The RABBIT crate layout. 


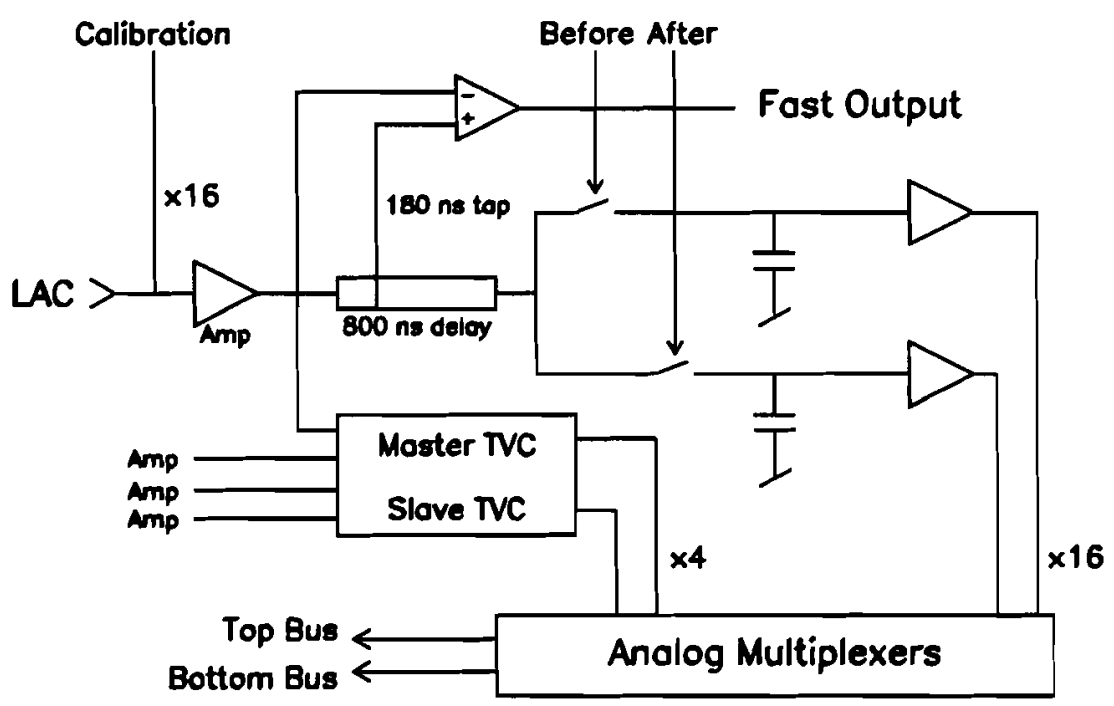

Figure 3.4: Block diagram of the LACAMP card. 
Fast Output, Sample and Hold and Time to Voltage Converter (TVC). The Fast Output signal was the difference between the amplified LAC signal and its $180 \mathrm{~ns}$ delayed copy; clipped signal was sent to the trigger circuitry. In case an event had been accepted by the trigger logic, two signals, Before and After separated by a programmable delay of $790 \mathrm{~ns}$, were sent from the BAT to the Sample and Hold modules of the LACAMP. The LAC signal was sampled at its baseline and peak. Both values were then available via the RABBIT backplane.

The purpose of the TVC module was to provide information about the time of arrival of the showering particle. Pulses from four adjacent LAC channels were added, and if the results exceeded a preprogrammed threshold ( 40 counts), charge started to buildup on a Master TVC capacitor . After $100 \mathrm{ns,}$, a second timing circuit (Slave TVC) became active in case another shower were to hit the same region of the LAC. Arrival of the Before signal stopped the charge build up on both capacitors and their voltages, proportional to the time that elapsed between the arrival of the shower and the Before signal, were made available for readout. All the amplifier and TVC channels could be independently calibrated and have their gains measured. This was done remotely using on board circuitry and timing sequences provided by the BAT modules.

\section{The EWE Module}

The function of the EWE was to enable communications between the modules of the RABBIT crate and the LAC DA system housed in CRATE-11 as well as to digitize signals available on the backplane of the RABBIT crate using on-board 16 bit ADC. It took $17 \mu$ s to digitize a signal from one amplifier or TVC channel.

The digital section of the EWE consisted of eight 16-bit registers. To accomplish a write to one of them, a 24 bit word was sent from the FASTBUS CRATE-11 to the EWE. The lower 16 bits were the data bits while the upper 8 were control bits 
specifying the EWE register number. The functions of the most frequently used registers were as follows:

CONTROL REGISTER Its bit contents determined what action the EWE would take on receiving an EXE (execute) signal from an ICBM ${ }^{1}$. Some of the possible operations were:

xconv - digitize the signal available on the backplane

digop - perform a digital transfer across the backplane

idir - write to the backplane (read if the bit is set to 0 )

STATUS REGISTER Contained a set of flags. Some of them were:

bot bus - EWE controls the Bottom Bus

data - new data in the ADC register

done - last operation completed

CHANNEL ADDRESS Contained the slot number of the RABBIT crate and the channel address for the card residing in it. For each LACAMP card there are 24 channels available: 16 amplifier and 8 TVC channels.

PEDESTAL Set the value of the pedestal to be subtracted from the processed signal (the E706 value was $0 \mathrm{~V}$ ).

THRESHOLD Determined the EWE ADC range; E706 used ADC's operating between -1 and 9 Volts, and 6500 counts corresponded to $0 \mathrm{~V}$.

ADC Contained the result of the last digitization.

READ DATA Contained a byte read from the backplane

WRITE DATA Contained a byte to be written to the backplane.

\footnotetext{
${ }^{1}$ Unless stated otherwise, setting a bit to 1 meant take appropriate action.
} 


\section{The BAT Module}

Besides providing timing signals for the LACAMP modules, the BAT served as a crate monitor and terminator. As mentioned before, Fast Output signals were sent to the trigger circuitry; if an event had been accepted by the trigger, an EVENT signal was sent to every BAT, which caused the Before - After sequence to be sent to the LACAMP cards. In Calibration mode, the Before - After sequence was preceeded by two pulses, TCAL1 and TCAL2, which simulated the detection of the showers. The BAT module was also able to monitor power supply voltages, delays of the timing signals and the temperature of the RABBIT crate.

\subsubsection{The FASTBUS System}

The following electronic modules were used in the LAC readout process $[44,45,46]$ :

LECROY 1892 Multiple Record Buffer (memory card). The card could store 4MB of data in FIFO (First In First Out) mode. Modules could be connected in series to increase the size of the buffer.

LECROY 1810,1879 Calibration and Trigger Module, Pipeline Time to Digital Converter. These modules were used by the STRAWs readout. and were housed in separate FASTBUS crates. STRAWs memory modules were housed in CRATE-20.

LECROY 1821 Programmable Segment Manager/Interface. This module provided operations on the FASTBUS crate backplane. It could serve as an interface between two FASTBUS crates (CRATE-11 and CRATE-20) or be connected to the host (CRATE-20 and VAX) via a specialized interface card (personality card). 
GPM General Purpose Manager manufactured by Struck. This Motorola 68000 based computer was used as a FASTBUS crate controller. Its microcode was written and compiled on a VAX. In CRATE-11, upon receiving a READOUT signal from the trigger, the GPM broadcast a START SCAN signal to the ICBM modules. After receiving DONE signals from all the ICBMs, the GPM sent a pulse to the trigger logic to remove the busy signal. In CRATE-20, the GPM microcode stepped through the data stored in the TVC and LAC buffers in order to find blocks of data with the same event number. When a match was found the matched blocks were concatenated by the GPM and sent through the 1821 to the VAX. If no match was found among three consecutive blocks, an error message was sent to the user and the event was not stored on tape.

ICBM Intelligent Control and Buffer Manager (see Figure 3.5). Its central component was a Motorola 56001 Digital Signal Processor (DSP) [47] operating at 10 MIPS. The DSP allowed for operations on 24,48 and 56 bit words. Internally it had four parallel buses (16 bit address and three 24 bit data) and three memory areas $\mathrm{P}, \mathrm{X}$ and $\mathrm{Y}$; each with 512 locations. $\mathrm{X}$ and $\mathrm{Y}$ memories were subdivided into 256 words of ROM and 256 words of RAM. The ICBM had in addition 194k on-board RAM memory divided also into $P, X$ and $Y$ spaces. They were accessible to the DSP and FASTBUS through one address and one data bus. The lower $512 \mathrm{P}$ as well as $256 \mathrm{X}$ and $\mathrm{Y}$ locations were mapped into the corresponding DSP memory spaces. Upper locations of the $\mathrm{X}$ memory served as FASTBUS Control Status Registers (CSR0, CSR1,CSR7) and ICBM output buffer registers (buffers $0-3$ ). FASTBUS write into the CSR0 register was treated as an interrupt by the DSP. 


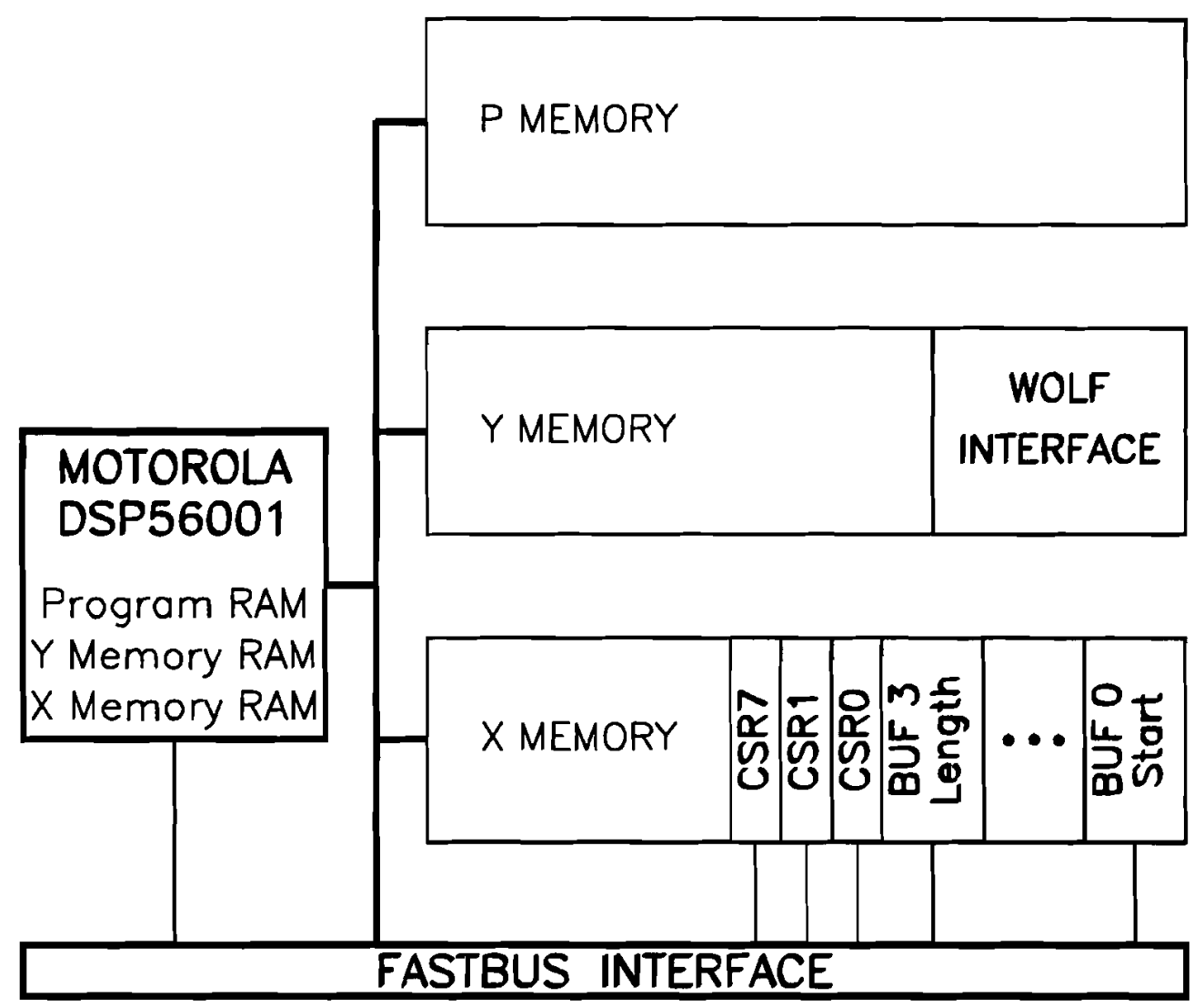

Figure 3.5: The ICBM block diagram. 
WOLF card designed for E706 provided the interface between the RABBIT system and the ICBM cards housed in CRATE-11. WOLF interface registers were mapped into the upper Y memory locations of the ICBM.

\subsection{The E706 Trigger}

The E706 trigger was designed to select events with high $p_{T}$ electromagnetic showers at rates up to $10^{6}$ interactions/second $[48,49]$. To acquire a large sample of interesting events populating the full $p_{T}$ range accessible to E706, a sequence of triggers was utilized. To provide data for the low $p_{T}$ end of the spectrum, prescaled triggers based on beam hodoscope and interaction counter information were used, the intermediate $p_{T}$ region was populated via prescaled LAC triggers with low $p_{T}$ thresholds, and the remaining part of the spectrum was selected by high threshold LAC triggers.

The amount of the transverse momentum seen in an event was inferred from the Fast Output signals of the LACAMPs. Energy $E_{i}$ deposited in the $i-$ th radial strip located $r_{i} \mathrm{~cm}$ from the center of the EMLAC contributed $p_{T i}=2 E_{i} \times r_{i} / z_{L A C}$ $\mathrm{GeV} / \mathrm{c}$ to the total $p_{T} ; z_{L A C}=900 \mathrm{~cm}$ was the distance from the target to the front of the EMLAC. For each octant an estimate of the deposited $p_{T}$ was made using custom $p_{T}$ adder cards, see Figure 3.6. This estimate was used to preselect event and to decide if the selected event would be written to the tape. Fast Output signals from pairs of the neighboring EMLAC strips were summed and attenuated using programmable DACs (attenuation meant multiplication by $2 r_{i} / z_{L A C}$ ). Such $p_{T}$ signals from 8 neighboring channels were summed to form so called 'sums-of8' (local sums). Local sums from the corresponding front and the back sections were fed into the discriminator modules to produce overlapping 'sums-of-16'. The results of the discrimination (individual 'sums-of-16' and their logical OR), called 


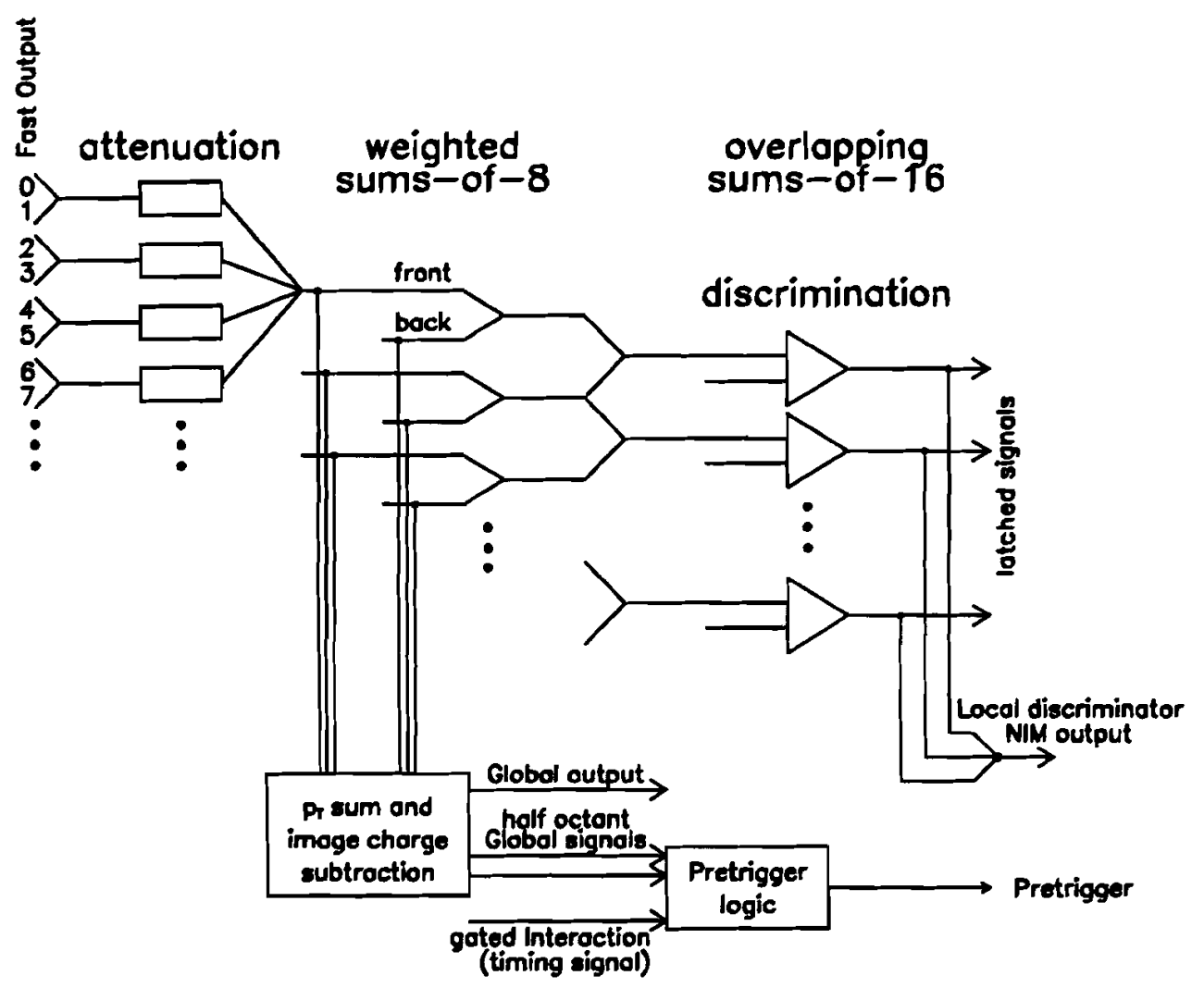

Figure 3.6: Local trigger signal formation. 
LOCAL signals, were then available to the trigger logic.

In addition, the following three global sum signals, prepared by $p_{T}$ cards, were used by the trigger logic:

- GLOBAL - the sum of all octant channels

- $1 / 2$ GLOBAL (inner) - the sum of the channels from the inner half of an octant

- 1/2 GLOBAL (outer) - the sum of the channels from the outer half of an octant

A modification to the $p_{T}$ adder cards was designed to overcome the image charge effect. Localized showers caused 'wrong sign' (positive polarity) signals in the neighboring strips due to the capacitive coupling between the $r$ boards and the lead plates. This could be a severe problem for global sums as a signal and its image overlapped significantly. Diodes were used to cut off wrong sign signals and the outermost $r$ strips were removed from global sums.

LAC information was used in the trigger formation only when the rest of the trigger logic decided that an interaction took place between a beam particle and the target. In what follows, I will explain the signals defining the presence of the beam particle and interaction, as well as the types of triggers used in the experiment.

The Tevatron delivered beam in $23 \mathrm{sec}$ spills separated by $35 \mathrm{sec}$ acceleration periods. The accelerator control provided three timing signals:

- BEGSP (begin spill) - the start of the spill.

- ENDSP (end spill) - the end of the spill.

- RF_CLOCK - the $53 \mathrm{MHz}$ pulser signal in phase with the bucket structure of the beam. 
The BEGSP and ENDSP signals were used by the trigger to generate the BMGATE (beam gate) pulse, setting the time interval during which the trigger logic was active. Two other gate signal generated by the trigger were:

- BUSY - disabled trigger during the processing of selected event.

- COMP_READY - DA system ready for data taking.

Two basic trigger signals, BEAM1 and LIVE_INT were defined as: BEAM1 = HODOSCOPE • BMGATE • RF_CLOCK LIVE_INT $=$ BEAM1 1 INTERACTION • CLEAN $\bullet \overline{\mathrm{BH}} \bullet$ COMP_READY

HODOSCOPE signal was true if there was a signal detected in adjacent paddles of at least two of the beam hodoscope planes. INTERACTION meant that at least two interaction counters fired, CLEAN condition that there was no hit in the interaction counters in the 3 preceding and the 3 following beam buckets and $\overline{\mathrm{BH}}$ that no in time signal was observed in the beam hole counter. These two signals did not contain any LAC based information and defined prescaled triggers. They also served as a first step in the formation of the LAC triggers.

For each LAC octant a PRETRIGGER signal was formed as logical AND of the following:

- LIVE_INT

- GLOBAL sum in the inner or outer section of the octant exceeds $1.7 \mathrm{GeV} / \mathrm{c}$.

- $p_{T}$ signal measured in the octant within previous $200 \mathrm{~ns}$ was less than $1.5 \mathrm{GeV} / \mathrm{c}$.

- no signal from the veto wall logic VW1 $+\mathrm{VW} 2 \cdot \mathrm{VW} 3$ in the relevant quadrant within $150 \mathrm{~ns}$ of trigger.

- no signal from the noise pickup of the RABBIT crates power supplies. 
The logical OR of octant PRETRIGGERs was sent to the BAT modules of the LAC and to the rest of the DA system to latch data for this event. A fraction of PRETRIGGER events was written to tape ${ }^{2}$. In the formation of the final trigger more detailed LAC data were used. For each octant three signals prepared by the $p_{T}$ boards (GLOBAL, 1/2 GLOBAL and LOCAL) were discriminated (using HIGH and LOW thresholds) and the following triggers were formed:

LOCAL GLOBAL HIGH = LOCAL LOW•GLOBAL HIGH

LOCAL GLOBAL LOW = LOCAL LOW•GLOBAL LOW

SINGLE LOCAL HIGH = LOCAL HIGH

SINGLE LOCAL LOW = LOCAL LOW

LOCAL 1/2 GLOBAL HIGH = LOCAL LOW•1/2 GLOBAL HIGH

TWO GAMMA $=\sum_{i}\left(\right.$ LOCAL LOW $\bullet \sum_{j}$ LOCAL LOW $)$

where $j$ is any of three octants opposite to octant $i$.

If at least one of those signals was generated, an interrupt would be sent to the DA. If none were satisfied, a reset signal was sent and after $20 \mu$ s the trigger was ready to accept a new event. Table 3.1 presents a summary of the 1990 trigger parameters.

\subsection{Event Readout}

The readout of the LAC data and the monitoring of the LAC readout system was done under the control of the LACSERVER program [50]. LACSERVER was run on the VAX computer connected to CRATE-11. It was able to communicate with RUN CONTROL, on-line monitoring programs and UPDATE. UPDATE was responsible for supplying LACSERVER with ICBM software ${ }^{3}$ and for archiving the

\footnotetext{
${ }^{2}$ If PRETRIGGER signal was false, the DA was reset.

${ }^{3}$ LACSERVER loaded LAC DA software into the ICBM P memories (LACRDOUT program into slaves and ICBMCNT into the master ICBM, both written in the DSP assembler) and LAC channel address tables into the slave ICBMs $\mathrm{X}$ memories.
} 


\begin{tabular}{|c|c|c|c|}
\hline Trigger Type & $\begin{array}{c}\text { Prescale } \\
\text { Factor }\end{array}$ & $\begin{array}{l}\text { Thyich } \\
\text { Threshold } \\
(\mathrm{GeV} / \mathrm{c})\end{array}$ & $\begin{array}{c}\text { Fraction } \\
\text { of Events (\%) }\end{array}$ \\
\hline LOCAL GLOBAL HIGH & 1 & 3.0 & 35 \\
\hline LOCAL GLOBAL LOW & 40 & 2.5 & 20 \\
\hline SINGLE LOCAL HIGH & 1 & 3.5 & 40 \\
\hline SINGLE LOCAL LOW & 40 & 3.0 & 18 \\
\hline TWO GAMMA & 1 & 2.8 & 20 \\
\hline LOCAL $1 / 2$ GLOBAL HIGH & 1 & 3.0 & 35 \\
\hline PRETRIGGER & 2925 & 1.7 & 7 \\
\hline BEAM & $15^{6}$ & - & 2 \\
\hline INTERACTION & $15^{5}$ & - & 3 \\
\hline DIMUON (672) & 1 & - & 20 \\
\hline
\end{tabular}

Table 3.1: 1990 trigger characteristics. 
calibration data. Upon receiving a BEGIN_RUN signal from RUN CONTROL, LACSERVER initialized a sequence of LAC calibration tasks (if requested by the operator). Approximately every 8 hours a LAC 'cold start' was performed at the beginning of a run. It reset the FASTBUS system, loaded ICBM software (if necessary) and collected data concerning the status of the LAC electronics. After completion, RUN CONTROL initialized the DA processes on PDPs and then the DA system was ready to accept triggers. During the spill, the GPM was responsible for initializing the event readout and coordinating data transfer from the ICBM buffers to the memories in CRATE-20. In between spills LAC monitoring tasks were performed under the control of LACSERVER and the master ICBM. Their results were compared with calibration data so that equipment malfunction could be promptly detected. After receiving an END_OF_RUN signal from RUN CONTROL, LACSERVER sent a global END_OF_RUN at the beginning of the following spill. It ended the DA processes on the PDPs.

\subsubsection{LAC Event Readout Tasks}

Readout of the LAC channels was accomplished by the FASTBUS based system housed in CRATE-11. Under the control of the GPM module, digitized LACAMP signals were read by ICBM cards and transferred to the memory modules in CRATE-20. CRATE-20 GPM performed a concatenation of the LAC and STRAWs data and made them available to the host VAX for concatenation with information from the PDPs.

LAC tasks, macros of the LACRDOUT program, were selected by a flag set by LACSERVER or the GPM in the CSR1 register. Each of the slave ICBMs executed the task independently and its completion was signaled by setting the DONE bit. The logical AND of DONE bits or DONE issued by the master ICBM in the case of some tasks, signaled the completion of task to LACSERVER or the GPM. Tasks 
controlled by the master ICBM were: LACAMP pedestal task, LACAMP ADC and TVC gains task, BAT monitoring task, and trigger gains task.

New versions of the LACRDOUT program were tested by reading out the channel addresses (instead of digitizing their contents) and comparing them with the numbers from the channel list. This option was incorporated into the DSP program and proved to be very helpful.

\section{LACAMPs Readout}

The broadcast selecting this task was made by the CRATE-11 GPM. The GPM also selected one of the four buffers to be filled with the event data. This number was read by ICBMs from the CSR0 locations. The master ICBM wrote into its buffer the event number, run number and timing information as provided by the HYDRA SCALER. Slave ICBMs performed the readout of the RABBIT crates in parallel.

The words frequently written to the EWE registers (channel addresses, digitize, read) were prepared on the VAX and stored by UPDATE in the ICBM memories. The TVC channel addresses were then copied into the internal DSP X-memory area to further reduce readout time. At each stage, four LACAMP addresses were selected (one for each EWE in both RABBIT crates), digitized in parallel and the results stored in the output buffer. During digitization, which took about $17 \mu \mathrm{s}$, a check was made if four previously stored channels belonged to the same TVC circuit. If it was true, and one of them (this time belonging to the same LACAMP card) exceeded the value of its calibration pedestal value by more than 80 counts, a flag was set to read out the corresponding master and slave TVC. All LACAMP channels were digitized in each event and all data were written to the output buffer. TVC readout was performed for EMLAC channels only. Flagged TVC channels were digitized and the results appended to the end of the output buffer. 
To interpret TVC data correctly, each word was accompanied by the TVC channel address from the list stored in the ICBM Y memory. Later, at the reconstruction stage, the TVC channel address was used to associate timing information with the appropriate EMLAC showers. The length of the buffer was then determined, written into the proper $\mathrm{X}$ memory location and the DONE bit was set in CSR0. The logical AND of DONE signals from slave ICBMs signaled the completion of the task and upon receipt of that signal, the DA system was ready for accepting new triggers. Under the supervision of the GPM, the contents of the ICBM buffers were transferred to CRATE-20. If a new trigger appeared during the transfer, the GPM would stop the process, fill the next buffer with the new event and then resume the transfer of the previous buffer.

The time needed to read out an average event was about $12 \mathrm{~ms}$. To read out all the LACAMP and TVC channels $18 \mathrm{~ms}$ were needed.

\section{Pedestal Task}

The Pedestal task involved both master and slave ICBMs. First an EVENT signal was sent from the master ICBM to the BAT modules to initiate the Before - After timing sequence. Then a START SCAN was broadcast to the slave ICBMs, which caused all LACAMP channels to be read out and the data to be stored. After the completion of the readouts, 128 readouts during the 1990 run and 512 readouts during the 1991 run, pedestal mean values and standard deviations were calculated for each of the channels. This task was performed between the accelerator spills and as one of the 'cold start' tasks. 'Cold start' results were stored by UPDATE in the run constants data base as well as in a portion of ICBM X-Memory. Their values were used during the data taking to select TVC channels for the readout. The calibration pedestals were in disagreement with pedestals calculated using beam triggers [50] (events which did not require interaction of the beam particle with a 
target). The latter method was then used to prepare a revised set of pedestal run constants for reconstruction purposes (on-line pedestals were used for monitoring purposes). It gave stable pedestal values (run to run variation less then 2 counts) which were on average 4 counts smaller than the ones obtained on-line. Such a difference could change the energy of an average shower by $1 \%$. The typical pedestal value was 6400 counts and the RMS varied between 15 counts for the front section of the HALAC to 22 counts for the back section of the EMLAC.

\section{Gains Task}

Amplifier and TVC channel gains were measured for all the LACAMP cards. This was done by taking 8 readings at each of the 6 calibration settings. Linear fits were than made and gain values extracted.

\section{BAT Task}

The BAT task monitored the RABBIT crate voltages, temperatures and timing signals used in the calibration and readout tasks. All those data were accessible to the ICBM through the BAT module.

\section{PROM Task}

Each RABBIT crate component was identified by its PROM number. It was important to keep track of LACAMP cards which were replaced for various reasons during the run, since each component had its specific characteristics (gains, properties of the calibration circuits).

\section{Path Check Task}

Each of the EWE registers was checked by writing and then reading back test commands. This task also checked the integrity of data transmission through the 
data cables and the status of the optical couplers. 


\section{Chapter 4}

\section{Event Reconstruction}

Unlike experiments with low frequency triggers which can have their data reconstructed on-line, the E706 data were not formatted for direct physics analysis. The tapes contained raw data, i.e. the results of digitization and discrimination operations assigned to electronic channel numbers. The first level of analysis was the reconstruction of the events. Digital data from various sources had to be translated into physical quantities: momentum, energy, charge, position. Results of the reconstruction were written to Data Summary Tapes (DST's) and then subjected to physics analysis.

The E706 event reconstruction code, called MAGIC [51], was written in FORTRAN - 77 within the PATCHY [52] code management system. It allowed for efficient code upgrades and maintenance. The ZEBRA [53] memory management system was used to provide dynamic memory allocation. MAGIC was implemented on DEC VAX, Silicon Graphics Indigo Workstations and IBM RISC 6000 machines and was able to write DST's in machine independent format.

The functions of the main reconstruction code were as follows:

- to read the raw data from the data tapes and run constants ${ }^{1}$ from the disk,

\footnotetext{
${ }^{1}$ Run constants were databases containing the information about channel pedestals, ID's of electronic components, and positions of the detectors in the experiment coordinate system. They were gathered during the cold starts of runs or evaluated off-line.
} 
- to unpack raw data and store them into ZEBRA memory banks,

- to call reconstruction packages for individual spectrometer components,

- to report errors,

- to write output to the DST.

Each major component of the E706 spectrometer was served by an independent reconstruction program. The programs relevant for this analysis were:

- DLREC (Discrete Logic REConstructor) - used for trigger logic and Cerenkov detector reconstruction,

- PLREC (PLanes REConstructor) - used for the tracking system reconstruction,

- EMREC (Electro-Magnetic REConstructor) - used for the reconstruction of the electromagnetic showers in EMLAC.

Other dedicated reconstructors dealt with the information from the hadron calorimeter (HCREC), the forward calorimeter (FCREC) and the E672 muon system (MUREC). In the following, I will present an overview of the reconstruction programs.

\subsection{DLREC}

The following information, from trigger electronics latches, was written to the DST's [49]:

- Four quality words containing information about the EMLAC trigger type, trigger performance, beam particle types, and discrepancies between components of the trigger logic. 
- An array containing detailed information about trigger types firing in EMLAC octants, time histories of the beam hodoscope components and interaction counters as well as Cerenkov detector data.

- An array (prepared within EMREC) containing the amounts of energy deposited in the groups of eight adjacent EMLAC strips. Those numbers corresponded to the analog sums of 8 channels formed by the trigger circuitry.

- Arrays containing discriminator thresholds.

The above information was used in forming cuts in the final data analysis (veto wall cut), in determining the inefficient trigger regions in the EMLAC, and in testing the performance of the triggers.

\subsection{PLREC}

PLREC reconstructed charged particle tracks using hits collected by the SSD beam and vertex chambers, the PWCs and the STRAWs [54]. Upstream (with respect to the position of the analysis magnet) tracks were formed from the SSD information, while PWCs and STRAWs provided the information to reconstruct downstream tracks. The determination of the particle trajectories was done independently for the two sections. Upstream tracks were used to find primary and secondary vertices. The linking (correlation) of upstream and downstream tracks in the center of the magnet gave information about particle charges and momenta.

The basic algorithm used in view track reconstruction selected pairs of hits in the same view from two of the SSD or PWC planes (seed planes), determined the straight line through them, searched for hits close to the line in the remaining planes (search planes) and finally refitted a line using all hits. In the case of a system with more than 2 views (the PWCs) the algorithm correlated view tracks 
to obtain 3 dimensional space tracks. The selection of the best tracks was based on the number of hits used for fitting and on the final $\chi^{2}$ of the fit.

The reconstruction of beam tracks was based on the hits from the 3 upstream SSD chambers. The first and third of the $\mathrm{X}$ and $\mathrm{Y}$ planes served as seed planes, and the search window for associating hits from the middle plane was set to $75 \mu \mathrm{m}$. In forming 2 hit tracks, only hits not belonging to 3 hit tracks were used. The track with the smallest impact parameter with respect to the vertex was called the beam track.

The PWC and STRAW tracking resulted in the determination of 3 dimensional space tracks. The first 3 and 4 hit tracks were formed in all four views. Two passes were made alternating seed plane pairs in order to find all three hit candidates. Next, a space track candidate was defined using pairs of tracks from $\mathrm{X}$ and $\mathrm{Y}$ views. Its positions in the $\mathrm{U}$ and $\mathrm{V}$ views were determined and a search for hits within 1.5 wire spacing was made. The procedure was then repeated with $U$ and $\mathrm{V}$ view tracks defining space track candidates. Candidates with at least 13 hits, good $\chi^{2}$ and a small number of shared hits were accepted as space tracks. Hits used to form them were excluded from a search for lower quality tracks such as ones with one or more PWCs missing. STRAW hits were used to improve the resolution of the PWC system. By projecting PWC space tracks into the STRAW planes, finding closest hits and refitting using information from all 32 planes the space track resolution improved from $1.25 \mathrm{~mm}$ to $0.5 \mathrm{~mm}$.

Vertex SSD's 5 and 4 hit view tracks were formed and projected to the center of the magnet. Then, they were linked with projected downstream space tracks. A momentum dependent matching window was used to associate up to 5 upstream tracks with one space track. 3 hit SSD tracks were also formed using hits remaining after 4 and 5 hit tracking. They were kept only if they linked to space tracks without 4 or 5 hit companions. 
In finding the position of the vertex, preference was given to linked 3,4 and 5 hit view tracks. At least three tracks were needed to find the vertex position by minimizing the following $\chi^{2}$ function:

$$
\chi^{2}=\sum_{\text {tracks }} \frac{b_{i}^{2}}{\sigma_{i}^{2}}
$$

for $\mathrm{X}$ and $\mathrm{Y}$ view tracks. $b_{i}$ and $\sigma_{i}$ were $i$-th track impact parameter and uncertainty in its projection. The $\mathrm{Z}$ position was a weighted average of the view tracks' $\mathrm{Z}$ coordinates. The final $\mathrm{Z}$ position was a weighted average of $\mathrm{Z}$ 's from both views. The vertex resolution was $400 \mu \mathrm{m}$ for the longitudinal and $10 \mu \mathrm{m}$ for the transverse position. The vertex position was then used to relink the downstream and upstream tracks and to determine their physics parameters.

\subsection{EMREC and HCREC}

EMREC dealt with the reconstruction of showers detected in the EMLAC. Showers in each of the quadrants were reconstructed independently. After identifying groups of strips with signals above a predetermined threshold in the $r$ and $\phi$ views, a 2 parameter function (shower shape) was used to find the energy and position of each of the showers. As approximately half of the energy of the showering particle was deposited in $r$ strips and another half in $\phi$ strips, $r$ and $\phi$ showers with similar energies were defined as one shower and the information about detected particles ( $r$ and $\phi$ coordinates, energy, time of the arrival etc.) was written to the DST [55].

The hadron reconstructor (HCREC) did not make direct use of the shower shape concept. The energy of the hadron was determined by summing energies deposited in separate clusters of pads corrected for energy in the shower tails. Correction formulae had been obtained from the analysis of the effect of the variable threshold on the reconstructed energy of the hadron. The $X$ and $Y$ positions of the showers were averages of the $x$ and $y$ positions of the pads weighted by the 
pad energies. Detailed description of the HCREC can be found in [56].

\subsubsection{The Electromagnetic Shower Shape}

As mentioned above, the method used to reconstruct EMLAC showers was more sophisticated than the one used for the showers detected in the HALAC. A better precision was needed in determining the shower parameters, especially in the case of overlapping showers, in order to be able to distinguish between photons coming from a meson and direct photons. For this purpose, a function describing the average electromagnetic shower was generated based on Monte Carlo simulation [57]; 2000 full photon showers with a $1 \mathrm{MeV}$ cutoff on the energy of a shower component, were generated ${ }^{2}$. Using energies deposited in the radial strips of the calorimeter and the known position of the incident Monte Carlo particle, so called integral shower shape functions were determined for the front and back sections of the detector. The fraction of the total deposited energy contained in strip $i$ was plotted against $r_{i}$ which was the distance of the $i$-th strip center to the known position of the shower. Then both shapes were fitted using the following trial functions:

$$
\begin{aligned}
S_{\text {front }}(r) & =\frac{1}{r} \times\left(f_{1} e^{-f_{2} r}+f_{3} e^{-f_{4} r}+f_{5} e^{-f_{6} r}\right) \\
S_{\text {back }}(r) & =b_{1} e^{-b_{2} r}+b_{3} e^{-b_{4} r}+b_{5} e^{-b_{6} r}
\end{aligned}
$$

where $r$ was the distance from the shower center.

Each of the functions was normalized to unity; the parameters obtained by this method can be found in [57]. The shower shape function, $S_{\bullet}$, used in the EMREC program was defined as:

$$
S_{\text {s }}(r)=0.7 \times S_{\text {front }}(r)+0.3 \times S_{\text {back }}(r)
$$

\footnotetext{
${ }^{2}$ E706 events were fully simulated using the GEANT package for the spectrometer description and HERWIG as the event generator.
} 
These weights were obtained by optimizing the shower shape function to the data. The shower shape function turned out to be independent of the shower energy to better than $1.5 \%$.

\subsubsection{The EMREC Algorithm}

An unpacker subroutine put the data used by EMREC (digitized signals from EMLAC amplifiers and TVC channels) into ZEBRA raw data banks. Known dead channels were flagged and the following operations were performed on the live channel signals:

- Conversion from ADC counts to energy units (3.1 MeV per count). The energy scale of the EMLAC was determined [50] by adjusting the mass of low $p_{T}$ reconstructed $\pi^{0}$ and $\eta$ mesons to the world averages found in [9]. In the course of this procedure it was found that the reconstructed masses were functions of the number of beam days. Thus a time dependent adjustment was made to the above conversion rate.

- Pedestal subtraction. For some events, pedestal subtraction left residual effects characterised by global ramps and offsets present in $r$ and $\phi$ views. This was attributed to the image charge effect and the nonuniformity of the beam extracted from the Tevatron. An attempt was made to correct this behavior by fitting the event background in the $r$ views and subtracting the obtained function from the channels contents. $\phi$ view corrections were inferred from the behavior of the $r$ view background, since the smaller number of strips made the $\phi$ view fits unreliable [58].

- Channel contents, after pedestal subtraction, were corrected for the individual amplifier gains. 
Flagged dead channels and pairs of adjacent dead channels were filled with values obtained from exponential interpolation between their live neighbors [59]. The choice of the function was motivated by the shape of an average shower. In cases where the inner flash strip was dead (only one live neighbor available), the approach was different. The imbalance between the energies deposited in the inner part of the $r$ view and the corresponding $\phi$ region was found and assigned to the dead flash strip. The reason was that shower energies should have been distributed evenly between the views and a large imbalance would jeopardize the correlation process for the whole octant. Showers in the flash strips and in strips adjacent to them were later excluded from the analysis by a fiducial cut.

\section{Groups, Peaks and Gammas}

After those preparatory steps, the summed section of the EMLAC was formed by adding corresponding strip contributions from the front and back sections. A search was made for groups of adjacent strips whose energies exceeded $80 \mathrm{MeV}$ (95 $\mathrm{MeV}$ for the outer $\phi$ view). Groups were accepted for further analysis if :

- the energy deposited in at least one of the strips was greater than $300 \mathrm{MeV}$ (350 $\mathrm{MeV}$ in the outer $\phi$ view),

- the energy deposited in all the strips was greater than $600 \mathrm{MeV}$,

- the size of the group (number of strips above threshold) was greater than 3 ( 2 in the outer $\phi$ view)

The only exception to these rules was the case of a group adjacent to the inner edge of the $r$ view. In such a case, even a one strip wide group was accepted provided that significant energy was deposited in the wide inner-flash strip.

Any group of strips with energy above threshold could be due to one or more electromagnetic or hadronic showers. To identify them, another search for so called 
peaks was made. Each of the groups was scanned from the left to the right edge in search for local minima and maxima ${ }^{3}$. As soon as a local maximum bounded by two minima was found (in EMREC jargon, a peak and two surrounding valleys), its significance was checked. If the height of the peak with respect to the valley levels was consistent with strip energy fluctuations within 2.5 standard deviations ${ }^{4}$, information about the maximum was discarded and the search continued for another peak $^{5}$. If a peak was considered significant, another search was performed, this time in the front section only. The goal of this search was to determine whether the peak in the summed section consisted of one or possibly more showers. If additional maxima were found in the front section, valleys for each of them were reevaluated and the peak significance checked. For surviving peaks, an additional search for maximum in the back section was made.

In special situations in which only one peak had been found in the front (or there was only one peak in the group) and its energy, estimated as the energy in the strips between the valleys, was above $25 \mathrm{GeV}$, a search for so called shoulders took place. Two showers, for example from the 2 photon decay of a very high energy $\pi^{0}$, could be so close together that there would be no significant valley between the peaks, but there could be a 'bump' on one of the slopes of the peak. Its presence was inferred from the behavior of the logarithmic derivative of the strip energy with respect to the strip distance from the peak position. Since the shape of the ideal shower (its core in particular) was to a good approximation exponential, a tight overlap (seen as a 'bump') would produce a peak in the logarithmic derivative of the energy deposition. Again peak significance was checked and peak position was defined by the location of the logarithmic derivative peak.

\footnotetext{
${ }^{3}$ Special care was taken to identify maxima on the group boundaries.

${ }^{4}$ See Equation 4.9 for the definition of $\sigma^{2}$

${ }^{5}$ For broad, fluctuating profiles, the highest strip was defined as the peak and the valleys were positioned at the group edges.
} 
At this point all the parameters needed to define peak properties were determined. The numbers of the peak and valley strips were stored along with estimates of the shower positions in both sections. The latter were made using following formula:

$$
\text { peak_position }=1.7 \times \frac{E_{l}-E_{r}}{E_{l}+E_{r}}
$$

where $E_{l}, E r$ were energies of the strips to the left and to the right of the peak strip and peak_position was measured with respect to the center of the peak strip. Peak energies in the front and summed sections were found by summing energies of strips between the valleys. If there was no peak found in one of the sections, a zero flag was used for both the energy and the position. For each of the showers, two additional variables were defined: $E_{f} / E_{t}$ ratio and directionality ${ }^{6}$. The definition of $E_{f} / E_{t}$ was:

$$
E_{f} / E_{t}=\frac{\text { peak energy in the front section }}{\text { peak energy in the summed section }}
$$

It was an estimate of the longitudinal shower development; small values of $E_{f} / E_{t}$ indicated a shower with hadronic characteristics. Directionality, for $r$ view peaks only, was defined as

$$
\operatorname{dir}=R_{f}-\frac{Z_{f}^{L A C}}{Z_{b}^{L A C}} R_{b}
$$

where $R_{f}, R_{b}, Z_{f}^{L A C}$ and $Z_{b}^{L A C}$ were radial positions of the shower and $z$ coordinates of the first EMLAC cell in the front and in the back sections. For showers originating in the target area, the dir value would be in the vicinity of zero, but for example for muons travelling parallel to the beam and not rejected by veto walls its value would be large and positive thus providing an additional analysis cut.

Having found peaks and estimated their energies and positions, in the next step we determined these two parameters precisely by fitting the shower shape function

\footnotetext{
${ }^{6}$ For nonzero front and back positions only.
} 
to the peaks? ${ }^{7}$ Such fitted peaks were called 'gammas' in the EMREC program. In the single peak case, we searched for the minimum of $\chi^{2}$, defined below, with respect to a shower energy $E$ expressed in $\mathrm{GeV}$ :

$$
\chi^{2}=\sum_{i} \frac{\left(E_{i}-z_{i} \times E\right)^{2}}{\sigma_{i}^{2}}
$$

The sum extends from the first to the last strip in the group. $E_{i}$ is $i$-th strip energy, $z_{i}$ is the shower shape estimate of the fraction of shower energy deposited in the $i$-th strip and $\sigma_{i}^{2}$ is a function describing the resolution of the EMLAC which was found to be:

$$
\sigma_{i}^{2}(E)=(0.22)^{2}+(0.16)^{2} \times E+(0.01)^{2} \times E^{2}
$$

The procedure for fitting shower shape to the $r$ view peaks was unambiguous. The case of the $\phi$ view was complicated by the fact that since initially the radial position of the shower was not known, the width of the $\phi$ strips was undetermined and the $z_{i}$ fractions from Equation 4.8 were not easy to find. An estimate of the radial position based on the shower width was made so that the fitting procedure could be applied. The radial positions of the $\phi$ showers were known only after the correlation step (for details see below). If the energy of the boundary strip exceeded the group defining threshold such a strip did not participate in the fitting process. There were three corrections defined for each shower:

- the boundary correction - an estimate of the fraction of the shower energy deposited outside the fiducial volume of the LAC,

- the tail correction - an estimate of the fraction of the shower energy deposited in strips lying outside the valleys,

- the view boundary correction - an estimate of the fraction of the shower energy which should be deposited in the complementary view. For example,

\footnotetext{
${ }^{7}$ The details of the procedure differ in the case of single and multiple peak groups.
} 
for a shower found in the inner portion of the $r$ view, the correction estimated the energy which should be found in the outer $\phi$ view.

For the case of multiple peak groups, we first looked for peak-shoulder pairs and improved the position and energy estimates of both peaks, keeping their summed energy as a constraint. If the result of this splitting between peak and shoulder was smaller than one strip width or if the splitting was smaller than 1.75 times the strip width and the summed energy of the pair was below $120 \mathrm{GeV}$, the shoulder

peak was discarded. The $\chi^{2}$ definition for multiple peak fitting was similar to Equation 4.8. The $\chi^{2}$ was minimized for all peaks with respect to the peak positions and energies.

\section{Correlations}

At this point, the energy and position of every peak had been estimated using data from one view only. In the remaining part of the program, the gammas from the $r$ and the $\phi$ views were correlated in order to find the positions (both $r$ and $\phi$ ) and the energies of the detected showers. The segmentation of the detector into left - right and inner - outer parts made the task easier. For example, $r$ gammas with radial positions $r<40 \mathrm{~cm}$ could be correlated with inner $\phi$ gammas only. For gammas in the corresponding regions of the octant, the correlation process used the gamma energies to perform the correlation. The structure of the EMLAC cells caused shower energies to split nearly evenly between the $r$ and the $\phi$ views. (One had to take into account fluctuations in the longitudinal development of the shower and the fact that the first readout board was of $r$ type [60]. This was especially important for low energy showers.) Two gammas were considered to be $r$ and $\phi$ projections of the same shower if their total, as well as front and back, energies were similar. The correlation process was repeated twice. After 
the first pass, when the supply of gammas available for correlation was exhausted or a large energy imbalance between the views prohibited matching, $\phi$ gammas that had been paired acquired a better estimate of their $r$ positions. They were refitted to determine their energies more precisely. A second correlation pass was performed and complete information about the reconstructed showers (in EMREC language called photons) was written to the DST.

There were many possible configurations in correlating gammas. The code used was designed to deal with specific configurations separately (i.e. using separate subroutines). These configurations are listed in Table 4.1 and Table 4.2. Analysis of the events for which the correlation process failed, for example leaving some of the gammas uncorrelated, showed that problems were caused by high noise in the LAC strips or showers hitting dead regions of the detector.

The simplest and most frequently encountered correlation type was the so called one to one type, which I will describe in more detail since the methods used were common to all the configurations. For each pair of yet uncorrelated gammas, a check was made to determine whether their positions in the $r$ and the $\phi$ views were compatible and detector boundary corrections were applied to them. The results were used to determine energies and expected values of energy fluctuations in the front and back sections The significance of the energy difference between $r$ and $\phi$ view was then calculated in units of $\sigma^{2}$ defined in Eq 4.9. If its value was within the current correlation window (the program looped over the correlation routines several times, increasing the width of the window at each step), we considered those gammas correlated (in EMREC language they produced a photon), flagged it and stored the photon information in the data bank. Once a gamma passed the correlation criteria, it was excluded from the remainder of the correlation process.

In the one to two and two to one correlation cases we looked for triplets consisting of one gamma from the $r(\phi)$ view and two gammas from the $\phi(r)$ view. 


\begin{tabular}{|c|c|c|c|}
\hline $\begin{array}{l}\text { Correlation } \\
\text { Type Code }\end{array}$ & $\begin{array}{l}\text { Number of } \\
\text { Photons }\end{array}$ & $\begin{array}{c}\text { Number of } \\
r \text { View Gammas }\end{array}$ & $\begin{array}{c}\text { Number of } \\
\phi \text { View Gammas } \\
\end{array}$ \\
\hline $\begin{array}{l}1 \\
2 \\
3\end{array}$ & $\begin{array}{l}1 \\
2 \\
2\end{array}$ & $\begin{array}{l}1 \\
1 \\
2\end{array}$ & $\begin{array}{l}1 \\
2 \\
1\end{array}$ \\
\hline 11,15 & 1,2 & 1 from left $r$ near & 1 from inner $\phi$ \\
\hline 12,16 & 1,2 & $\begin{array}{l}1 \text { from right } r \text { near } \\
\text { inner-outer boundary }\end{array}$ & $\begin{array}{l}1 \text { from inner } \phi \\
1 \text { from outer } \phi\end{array}$ \\
\hline 13,17 & 1,2 & $\begin{array}{l}1 \text { from left } r \\
1 \text { from right } r\end{array}$ & $\begin{array}{c}1 \text { from inner } \phi \\
\text { near octant boundary }\end{array}$ \\
\hline 14,18 & 1,2 & $\begin{array}{l}1 \text { from left } r \\
1 \text { from right } r\end{array}$ & $\begin{array}{c}1 \text { from outer } \phi \\
\text { near octant boundary }\end{array}$ \\
\hline 21,26 & 2,2 & $\begin{array}{l}2 \text { from left } r \text { one near } \\
\text { inner-outer boundary }\end{array}$ & $\begin{array}{l}1 \text { from inner } \phi \\
1 \text { from outer } \phi\end{array}$ \\
\hline 22,27 & 2,2 & $\begin{array}{l}2 \text { from right } r \text { one near } \\
\text { inner-outer boundary }\end{array}$ & $\begin{array}{l}1 \text { from innér } \phi \\
1 \text { from outer } \phi\end{array}$ \\
\hline 23,28 & 2,2 & $\begin{array}{l}1 \text { from left } r \\
1 \text { from right } r\end{array}$ & $\begin{array}{c}2 \text { from inner } \phi \\
\text { one near octant boundary }\end{array}$ \\
\hline 24,29 & 2,2 & $\begin{array}{l}1 \text { from left } r \\
1 \text { from right } r\end{array}$ & $\begin{array}{c}2 \text { from outer } \phi \\
\text { one near octant boundary }\end{array}$ \\
\hline
\end{tabular}

Table 4.1: The simplest EMREC correlation types. Types $15-18$ correspond to $11-14$, but two peaks are created after adding the views separated by the view boundary. In types $21-24$ the boundary photon belongs to a one or two peak group. 


\begin{tabular}{|c|c|c|c|}
\hline $\begin{array}{l}\text { Correlation } \\
\text { Type Code }\end{array}$ & $\begin{array}{c}\text { Number of } \\
\text { Photons }\end{array}$ & $\begin{array}{c}\text { Number of } \\
r \text { View Gammas }\end{array}$ & $\begin{array}{c}\text { Number of } \\
\phi \text { View Gammas }\end{array}$ \\
\hline $\begin{array}{l}31,36 \\
32,37 \\
33,38 \\
34,39\end{array}$ & $\begin{array}{l}3,4 \\
3,4 \\
3,4 \\
3,4\end{array}$ & $\begin{array}{c}1 \text { from left } r \\
1 \text { from right } r \\
3,4 \\
3,4\end{array}$ & $\begin{array}{c}3,4 \\
3,4 \\
1 \text { from inner } \phi \\
1 \text { from outer } \phi\end{array}$ \\
\hline $\begin{array}{l}41 \\
42\end{array}$ & $\begin{array}{l}3 \\
3\end{array}$ & $\begin{array}{l}2 \text { from left } r \\
2 \text { from right } r\end{array}$ & $\begin{array}{l}2 \\
2\end{array}$ \\
\hline 48 & 2 & $3 ; 1$ or 2 from left $r$ & $\begin{array}{c}1 \text { from inner } \phi \\
\text { near octant boundary }\end{array}$ \\
\hline 49 & 2 & $3 ; 1$ or 2 from left $r$ & $\begin{array}{c}1 \text { from outer } \phi \\
\text { near octant boundary }\end{array}$ \\
\hline 53 & 2 & $4 ; 2$ from left $r$ & $\begin{array}{c}1 \text { from inner } \phi \\
\text { near octant boundary }\end{array}$ \\
\hline 54 & 2 & $4 ; 2$ from left $r$ & $\begin{array}{c}1 \text { from outer } \phi \\
\text { near octant boundary }\end{array}$ \\
\hline 56 & 2 & $\begin{array}{c}1 \text { from left } r \text { near } \\
\text { inner-outer boundary }\end{array}$ & $3 ; 1$ or 2 from inner $\phi$ \\
\hline 57 & 2 & $\begin{array}{l}1 \text { from right } r \text { near } \\
\text { inner-outer boundary }\end{array}$ & $3 ; 1$ or 2 from inner $\phi$ \\
\hline 61 & 2 & $\begin{array}{l}1 \text { from left } r \text { near } \\
\text { inner-outer boundary }\end{array}$ & $4 ; 2$ from inner $\phi$ \\
\hline 62 & 2 & $\begin{array}{l}1 \text { from right } r \text { near } \\
\text { inner-outer boundary }\end{array}$ & $4 ; 2$ from inner $\phi$ \\
\hline
\end{tabular}

Table 4.2: Multiple gamma correlation types defined in the EMREC. 
If in one of the views centroids of the showers were separated by less than a strip, gammas would strongly overlap. The determination of the correlation quality was done as in the one to one case. In addition a single gamma would be refitted using two shower shapes with energies inferred from the orthogonal view. Such situations often happen for high energy $\pi^{0}$ s with low asymmetry. More complicated one to three and one to four cases happen sporadically. They are similar to the one to two situation but no refitting of the single gamma was attempted in such cases.

Another large category, in terms of the cases involved, were boundary type correlations dealing with situations in which one of the gammas was near the view boundary; in the $r$ view near the inner-outer boundary and in the $\phi$ view near the left-right octant boundary. Showers producing such gammas in one view corresponded to two gammas in the other view. To perform the correlation, it was necessary to use information from three views. For example near the $r$ inner-outer boundary both the $\phi$ views were involved. Events involving boundary gammas had priority in the correlation process. Windows for energy and position differences were allowed to open wider than for nonboundary cases. Situations corresponding to the nonboundary one to one, two to one and higher order cases are possible, as shown in Table 4.2 .

\subsection{FCREC and MUREC}

The bulk of the data written to the DST's came from the reconstructors described above. Smaller amounts of information were provided by the Forward Calorimeter and the MUon REConstructors. FCREC wrote out the amount of energy deposited in the FCAL sections and the $P_{x}$ and $P_{y}$ of the signal while MUREC (E672 event reconstructor) wrote out data related to the muon tracks (slopes and intercepts, 
$\chi^{2}$ of the fits etc.). 


\section{Chapter 5}

\section{Data Analysis}

The data from the E706 1990 run were divided into several trigger sets. Table 5.1 shows the run ranges of those sets and the number of processed events in each set. Within each set the trigger characteristics, and the corresponding trigger corrections, were similar. This study of the two photon decay of the $\pi^{0}$ is based on the analysis of the events from runs 7907-9434 that were selected by the SINGLE LOCAL HIGH (SLH) trigger whose threshold was set at $3.5 \mathrm{GeV} / \mathrm{c} p_{T}$. The invariant mass spectrum of two photon combinations

$$
m_{i j}=\left(2 E_{i} E_{j}\left(1-\cos \theta_{i j}\right)\right)^{1 / 2}
$$

where $E_{i}, E_{j}$ are photon energies and $\cos \theta_{i j}$ is the opening angle between them in the laboratory coordinate system, is shown in Figure 5.1. Clearly visible are the $\pi^{0}$ and the $\eta$ signals from two photon decays. The cuts applied to the data and relevant corrections will be described in this chapter; the production cross sections will be presented in Chapter 6 . 


\begin{tabular}{|c|c|c|}
\hline \hline $\begin{array}{c}\text { Trigger } \\
\text { Set }\end{array}$ & Run Range & $\begin{array}{c}\text { Events } \\
\text { Processed } \\
\text { (millions) }\end{array}$ \\
\hline \hline 1 & $9181-9434$ & 5.9 \\
2 & $8989-9180$ & 4.1 \\
3 & $8629-8988$ & 5.8 \\
4 & $8240-8628$ & 3.9 \\
5 & $8055-8239$ & 2.9 \\
$6 \mathrm{~A}$ & $7907-8054$ & 2.1 \\
$6 \mathrm{~B}$ & $7594-7906$ & 2.8 \\
7 & $7472-7593$ & 1.8 \\
\hline \hline
\end{tabular}

Table 5.1: 1990 trigger sets. 


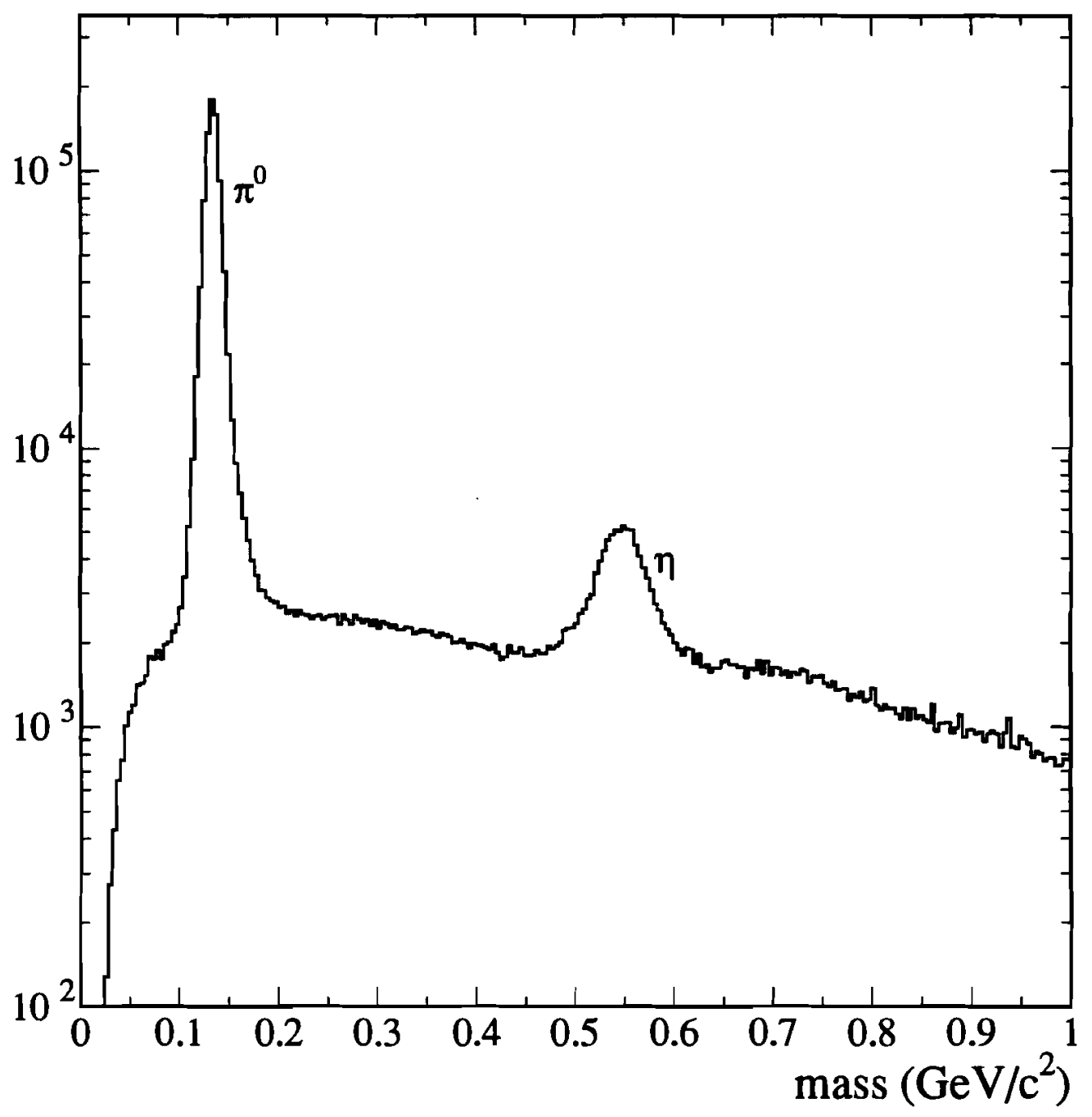

Figure 5.1: The two photon invariant mass spectrum for $p_{T}>4.0 \mathrm{GeV} / \mathrm{c}$ and $-0.75<y<0.75$. Cuts described in this chapter have been applied to obtain this distribution. 


\subsection{Data Selection}

\subsubsection{The Vertex Cut}

The $\mathrm{z}$ position distribution of the reconstructed vertices of events containing high $p_{T} \pi^{0} \mathrm{~s}$ is presented in Figure 5.2 a. Each entry has been corrected for beam absorption in the upstream material ${ }^{1}$ and photon conversions. Clearly visible are two pieces of copper, two pieces of beryllium and some of the beam and vertex SSDs.

The $\mathrm{x}-\mathrm{y}$ distributions of vertices are shown in Figures $5.2 \mathrm{~b}$ and $\mathrm{c}$. The acceptance edges of the instrumented part of the beam SSDs, the beam hodoscope and targets are also shown. An offset between the center of the target and the center of the hodoscope is visible; a fraction of the beam registered by scalers did not intercept the target. A fiducial region of the target was defined to properly normalize the cross sections. The position of the center of the Be target was determined as $(-1.12 \mathrm{~cm},-0.07 \mathrm{~cm})$ in the laboratory coordinate system, the right edge of the $\mathrm{Cu}$ was at $x=-0.12 \mathrm{~cm}$ and the hodoscope area of $2 \times 2 \mathrm{~cm}^{2}$ was centered at $(-0.4 \mathrm{~cm}, 0.0 \mathrm{~cm})$. The fiducial region of the target was an intersection of a circle with radius equal to $0.96 \mathrm{~cm}$ centered at the Be position, and the beam hodoscope. Vertices found in the upstream set of beam SSDs were used to determine the fraction of the beam hitting the target. The number of vertices within the target fiducial region divided by the number of vertices within the hodoscope coverage gave a target fiducial correction.

\subsubsection{The EMLAC Fiducial Volume}

One of the goals of the reconstruction process was to minimize the number of uncorrelated gammas found in the EMLAC. Even gammas close to the EMLAC

\footnotetext{
${ }^{1}$ Correction for the $\pi^{-}$beam absorption in the material present upstream from the vertex position was based on the data published by Carroll at al [61].
} 

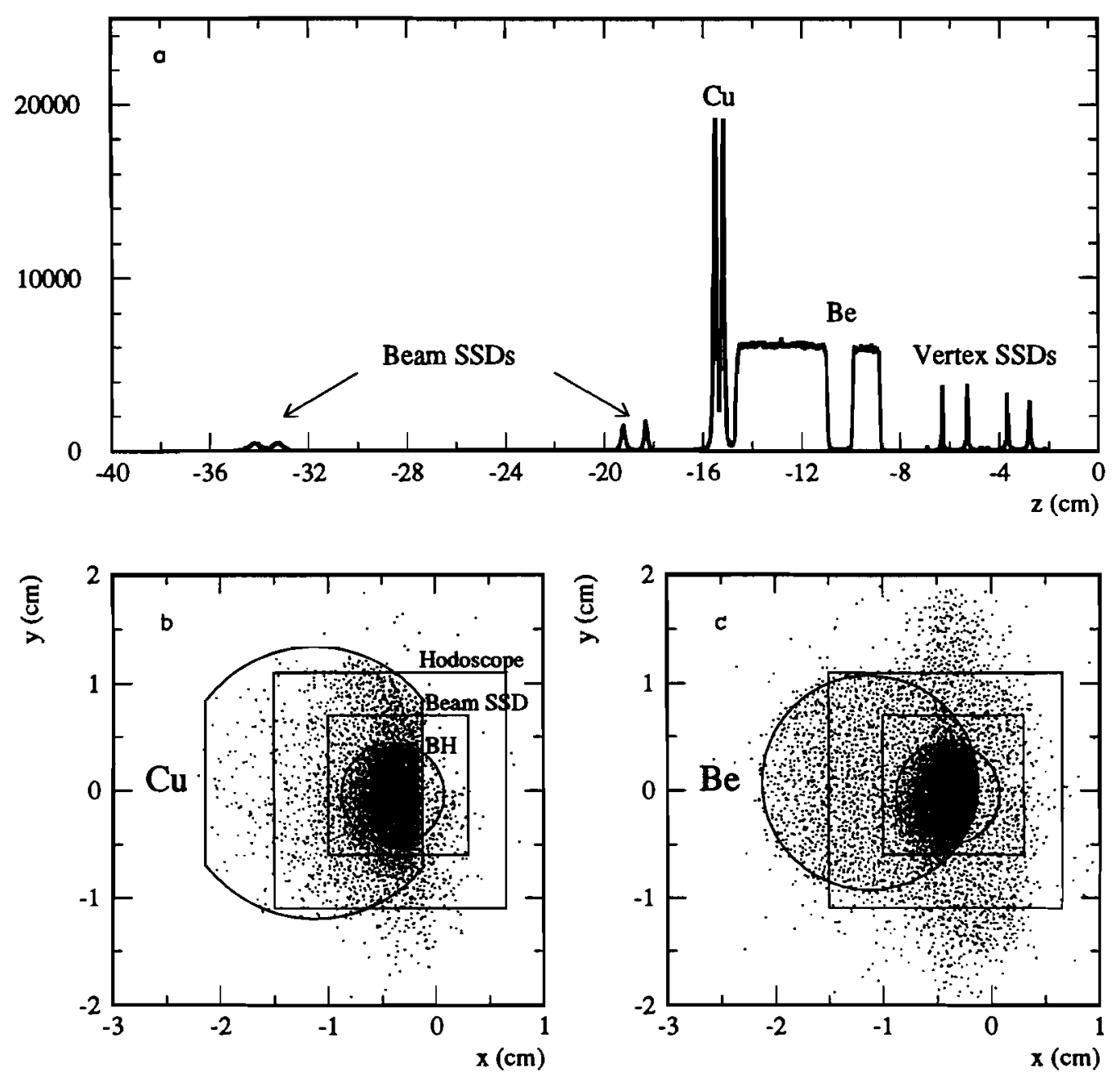

Figure 5.2: Unweighted distributions of the $\pi^{0}$ event vertices. a) The distribution along the $z$ coordinate axis. b) The $x-y$ distribution in the Cu targets. c) The $x$ - $\mathrm{y}$ distribution in the Be targets. 
boundaries were forced to correlate. In cases where a well defined shower peak was not found in one or both views, the final photon position was poorly determined. To exclude such poorly reconstructed photons from the further analysis, a fiducial cut was made on the photon positions. The condition was that the photon position should be at least $2 r$ strip widths away from the:

- inner edge of the octant (cut on the rapidity ${ }^{2}$ in the forward region)

- outer edge of the 235th $r$ strip, the last full EMLAC $r$ strip (cut on the rapidity in the backward region)

- octant and quadrant $\phi$ boundary (cut on the azimuthal coverage)

The distribution of the $\pi^{0}$ candidates whose photons satisfied the EMLAC fiducial volume cut is presented in Figure 5.3. The $\pi^{0}$ position was defined as the energy weighted average of photon positions.

The geometric acceptance correction was calculated using a simple Monte Carlo simulation. A number of $\pi^{0} \mathrm{~s}$ was generated on a $p_{T}-y$ grid and then decayed in the $2 \gamma$ mode. The ratio (in a given $p_{T}-y$ cell) of the number of photon pairs hitting the fiducial region of the EMLAC and producing a $\pi^{0}$ candidate to the total number of $\pi^{0}$ s generated was the geometric acceptance. A set of tables was prepared for specific positions of the decay vertex [62]. These tables were employed to find the acceptance for any vertex by interpolation. Sample plots of the averaged acceptance in several $p_{T}$ bins are shown in Figure 5.4. It was also found that some rapidity regions in some of the EMLAC octants were unsuitable for analysis due to trigger inefficiencies. Such events were discarded and corrected for by weighting signals from corresponding rapidity regions in the rest of the detector with a factor

\footnotetext{
${ }^{2}$ We do not distinguish between the rapidity $(y)$ defined by $\tanh y=p_{z} / E$ and the pseudorapidity $(\eta)$ defined by $\tanh \eta=\cos \theta$; since $m_{\pi^{0}}$ is negligible compared to $E, p_{z}=E \cos \theta$.
} 


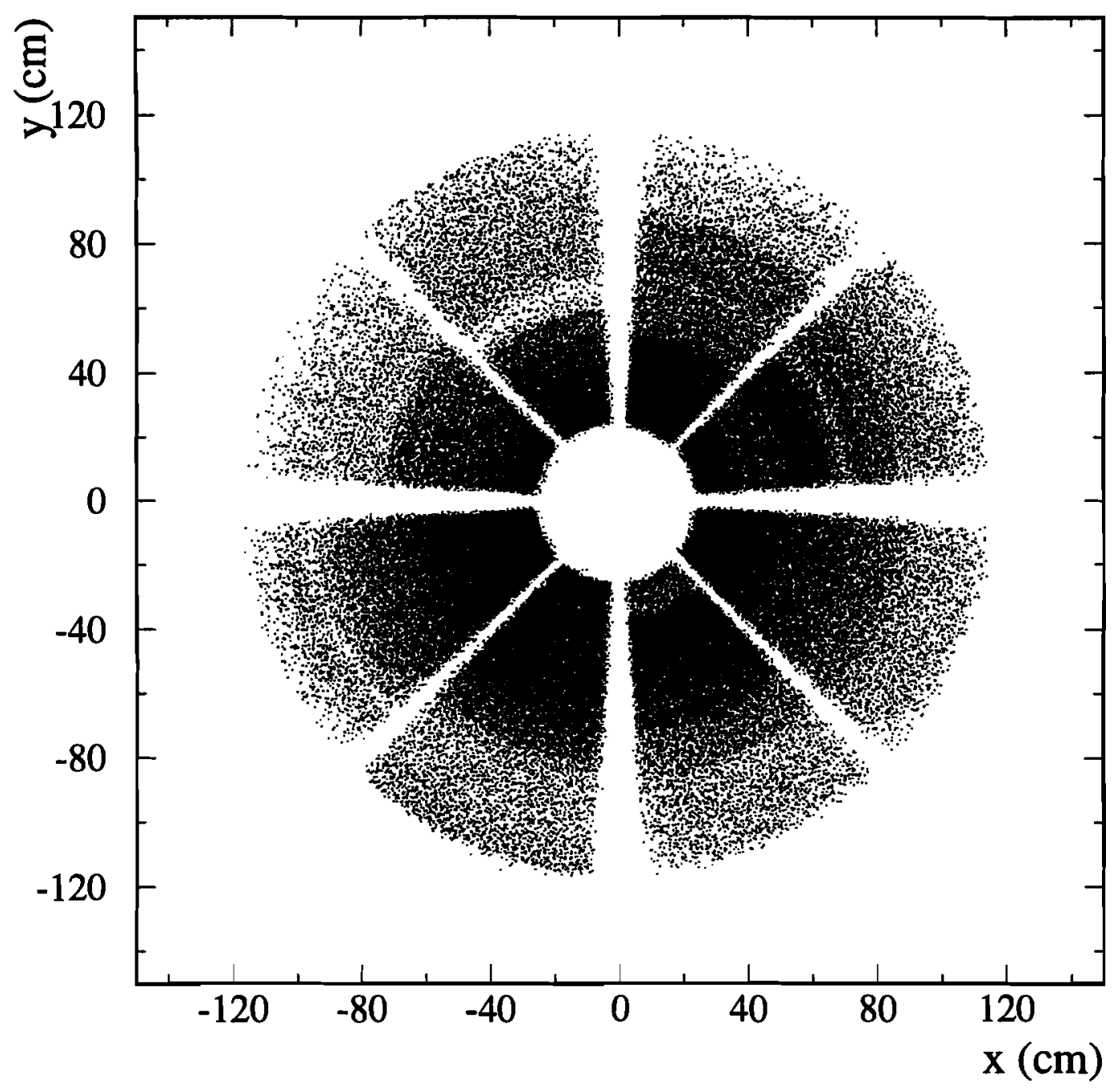

Figure 5.3: The distribution of the $\pi^{0}$ candidates within the EMLAC fiducial region. 

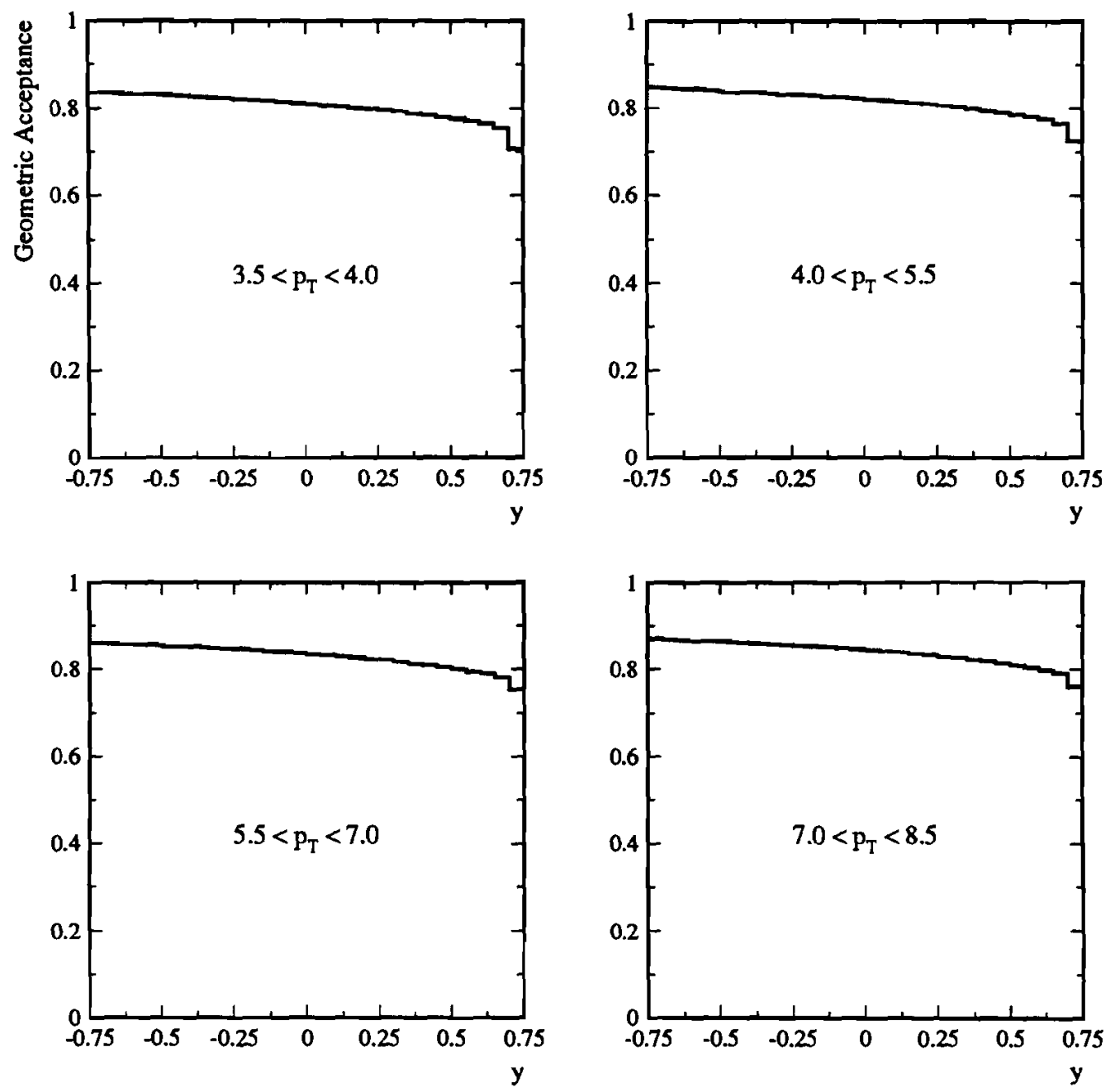

Figure 5.4: The averaged $\pi^{0}$ geometric acceptance function in selected $p_{T}$ bins. 
$w$ given by:

$$
w=\frac{8}{\text { number of octants with efficient trigger }}
$$

\subsubsection{The Energy Asymmetry}

Since the $\pi^{0}$ meson is a spin zero particle, the two photon decay in the $\pi^{0}$ center of mass is isotropic. The distribution of the reconstructed $\pi^{0} \mathrm{~s}$ as a function of the cosine of the decay angle $\theta^{*}$ relative to the direction of flight of the parent pion should be flat, and may serve as a test of the detector efficiency and the quality of the reconstruction process. The energy asymmetry A defined in the upper part of Figure 5.5 in terms of the photon energies in the laboratory system, is equal to $\cos \theta^{*}$ since the $\beta$ of the $\pi^{0}$ effectively equals 1 .

High asymmetry $\pi^{0} \mathrm{~s}$ may escape detection since the detector is insensitive to very low energy showers. The signal to background in the high asymmetry region is particularly poor, so a cut on asymmetry is invoked. The bottom part of Figure 5.5 shows the asymmetry distribution of the reconstructed $\pi^{0} \mathrm{~s}$. The entries

were calculated by extending the fit to the background under the $\pi^{0}$ mass peak $\left(0.1<m_{2 \gamma}<0.18\right)$ and subtracting it from the signal. The resulting distribution is nearly flat up to asymmetry equal 0.7 and drops to zero around 0.95 . The asymmetry cut value was set to 0.75 and the same value was used in the $\pi^{0}$ geometric acceptance and reconstruction efficiency calculations.

\subsubsection{Hadron Rejection}

Some of the showers reconstructed in the EMLAC were of hadronic origin. The criterion used to discriminate against these hadronic showers was based on the the $E_{f} / E_{t}$ ratio. Electromagnetic showers deposited the majority of their energies (approximately 70\%) in the front section of the EMLAC, while for hadronic showers the ratio was frequently closer to 0 . A cut, determined from the $E_{f} / E_{t}$ spectrum 

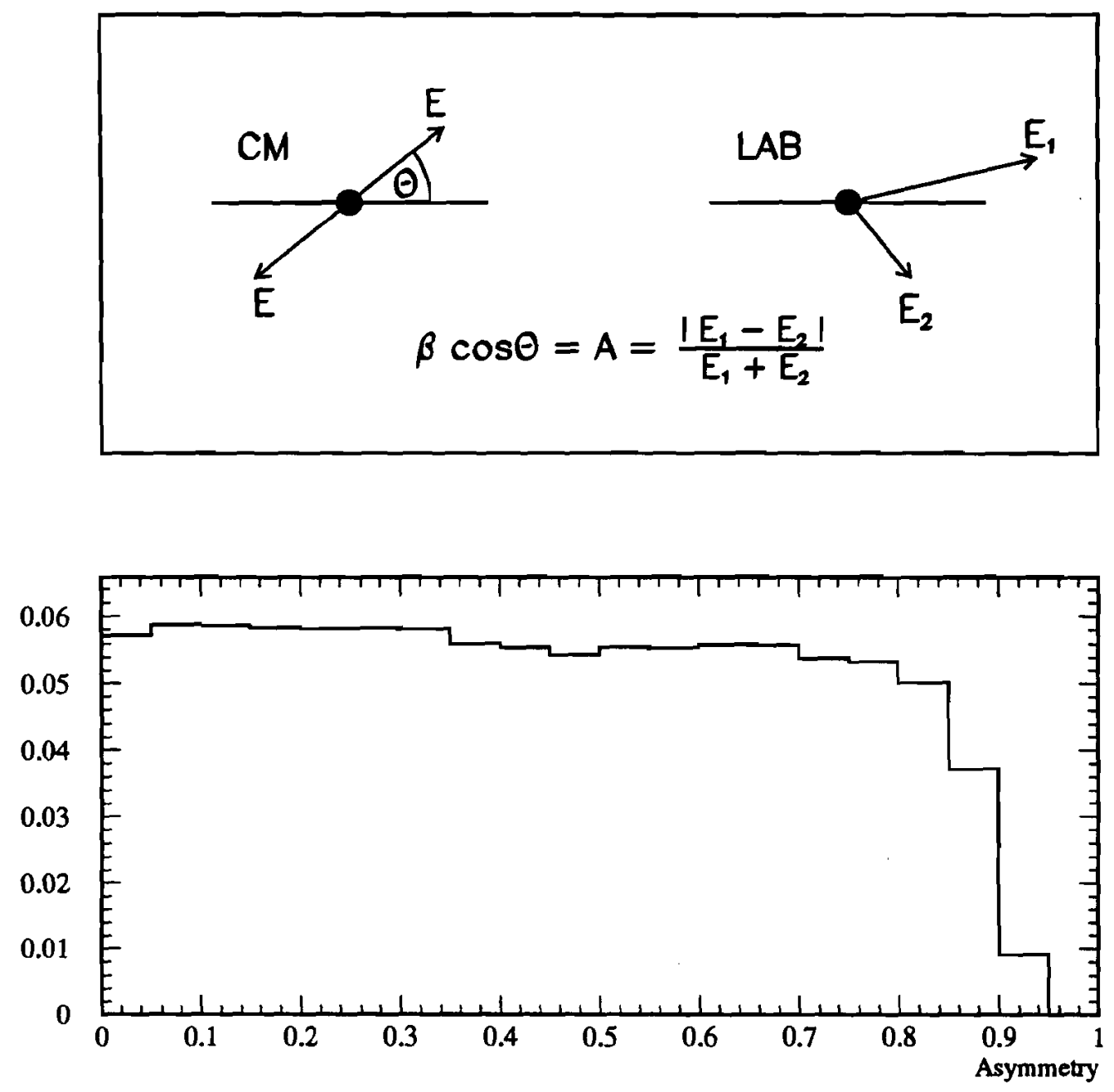

Figure 5.5: Top: The definition of the energy asymmetry. Bottom: The asymmetry spectrum of the reconstructed $\pi^{0} \mathrm{~s}$. 
was set at 0.2 , and the correction was incorporated in the Monte Carlo calculation of the $\pi^{0}$ reconstruction efficiency (see Section 5.2).

\subsubsection{Muon Rejection}

The two photon invariant mass distribution shown in Figure 5.1 is dominated by events from the lower end of the $p_{T}$ spectrum ${ }^{3}$. Similar plots made in $p_{T}$ ranges, as shown in Figure 5.6, reveal that the cuts made so far did not produce a clean pion signal above $p_{T}$ of $8 \mathrm{GeV} / \mathrm{c}$.

The background was mainly due to photons from muon bremsstrahlung which either combined with other showers present in the event or produced split showers due to the substantial shift between their front and back positions during the EMREC search for shoulders.

An off-line veto wall cut was set up to overcome the shortcomings of the on-line veto. For each EMLAC quadrant and for time bucket $i(3<i<13$ and $i=8$ corresponded to the trigger bucket) an expression $V W_{i}=(V W 1+V W 2) \bullet V W 3$ was evaluated where $V W 1, V W 2$ and $V W 3$ were TRUE if there was a signal from the corresponding veto wall quadrant within \pm 1 bucket. The logical oR of $V W_{i}$ 's for each quadrant was then formed. If the result was TRUE, the contribution from that quadrant for this event was removed from the analysis.

To further improve the signal to noise ratio, especially in the outer region of the detector, three more muon cuts were used: directionality, balanced $p_{T}$ and scaled $\chi^{2}$ [63]. The impact of all the cuts is shown in Figure 5.7.

The shower directionality has been defined in Section 4.3. This parameter was used to distinguish showers produced by particles emerging from the target region, having near zero directionalities, from showers caused by the beam halo and characterized by positive directionality values. The directionality cut was applied

\footnotetext{
${ }^{3}$ As shown in Chapter 6, the meson production cross section varies as $\sim p_{T}^{-9.3}$.
} 

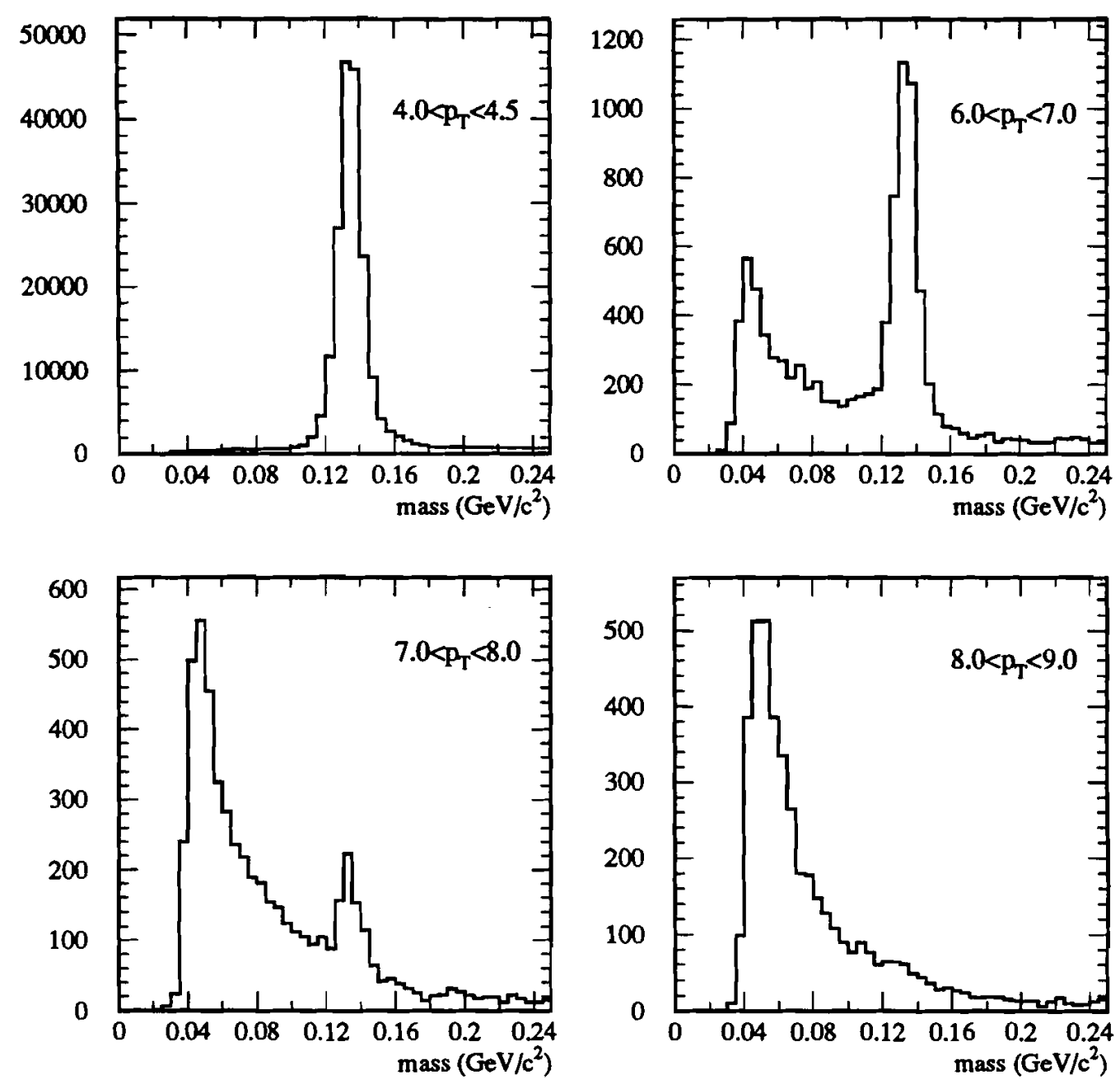

Figure 5.6: $\pi^{0}$ mass distributions after applying $E_{f} / E_{t}$ and asymmetry cuts. 

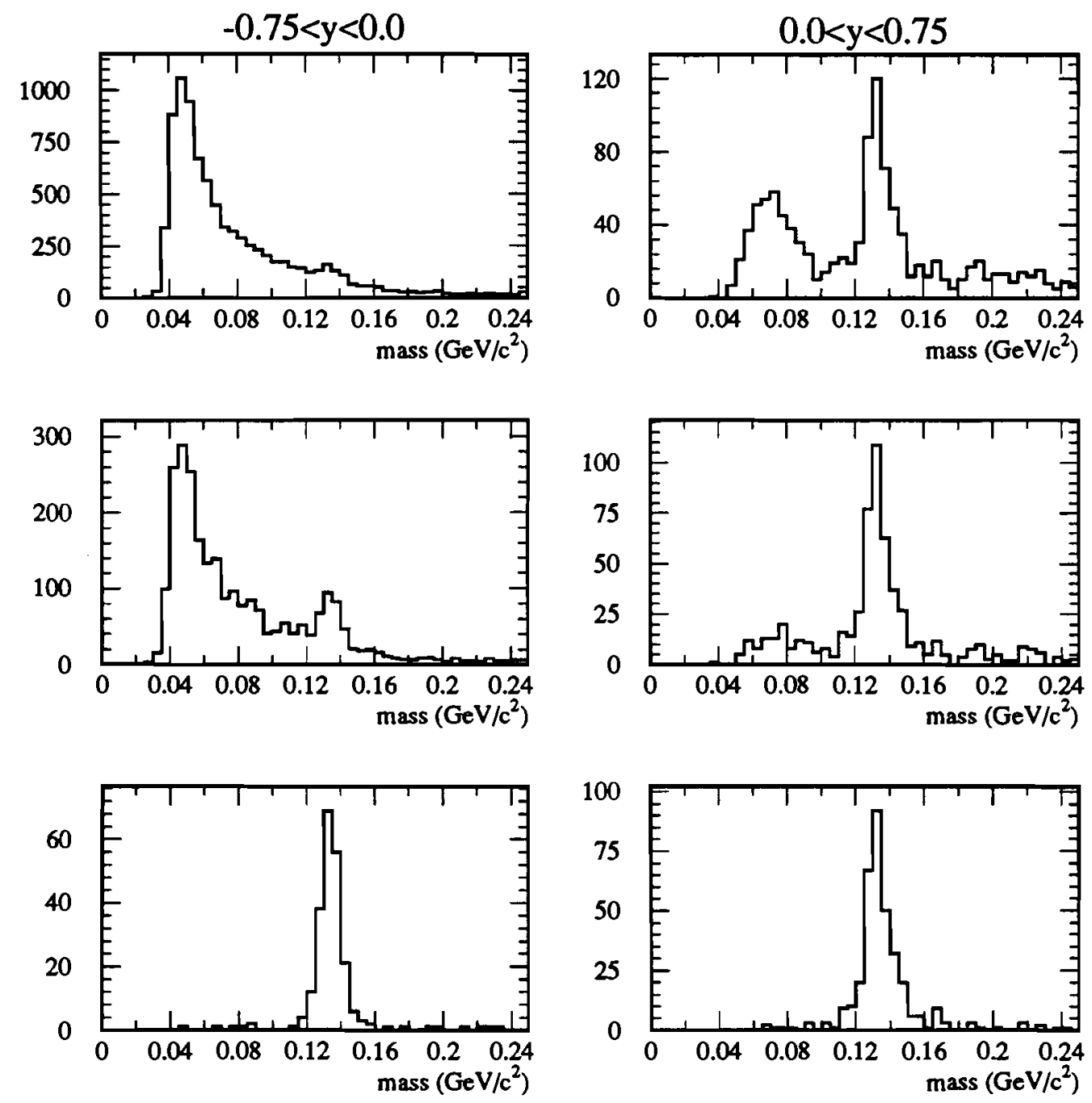

Figure 5.7: The impact of the veto wall and the muon rejection cuts on the $\pi^{0}$ mass distribution for $7.0<p_{T}<9.0 \mathrm{GeV} / \mathrm{c}$. Top row: distributions after $E_{f} / E_{t}$ and asymmetry cuts. Middle row: impact of the veto wall cut. Bottom row: impact of the additional muon rejection cuts. 

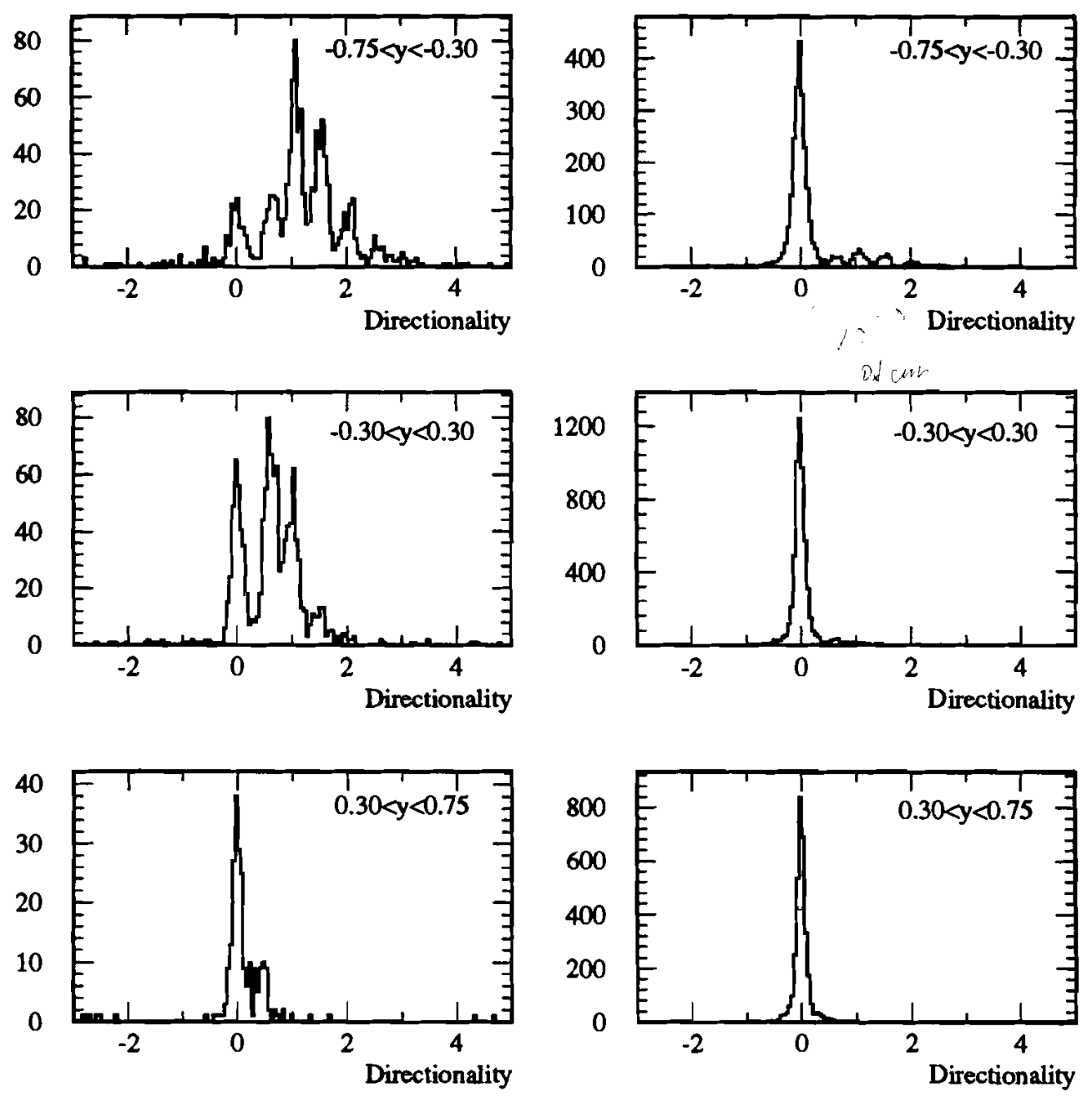

Figure 5.8: Photon directionality distributions in rapidity bins for $\pi^{0}$ with $5.5<$ $p_{T}<9.0 \mathrm{GeV} / \mathrm{c}$. Left column: events with the off-line veto wall signal. Right column: Events without the off-line veto wall signal. 

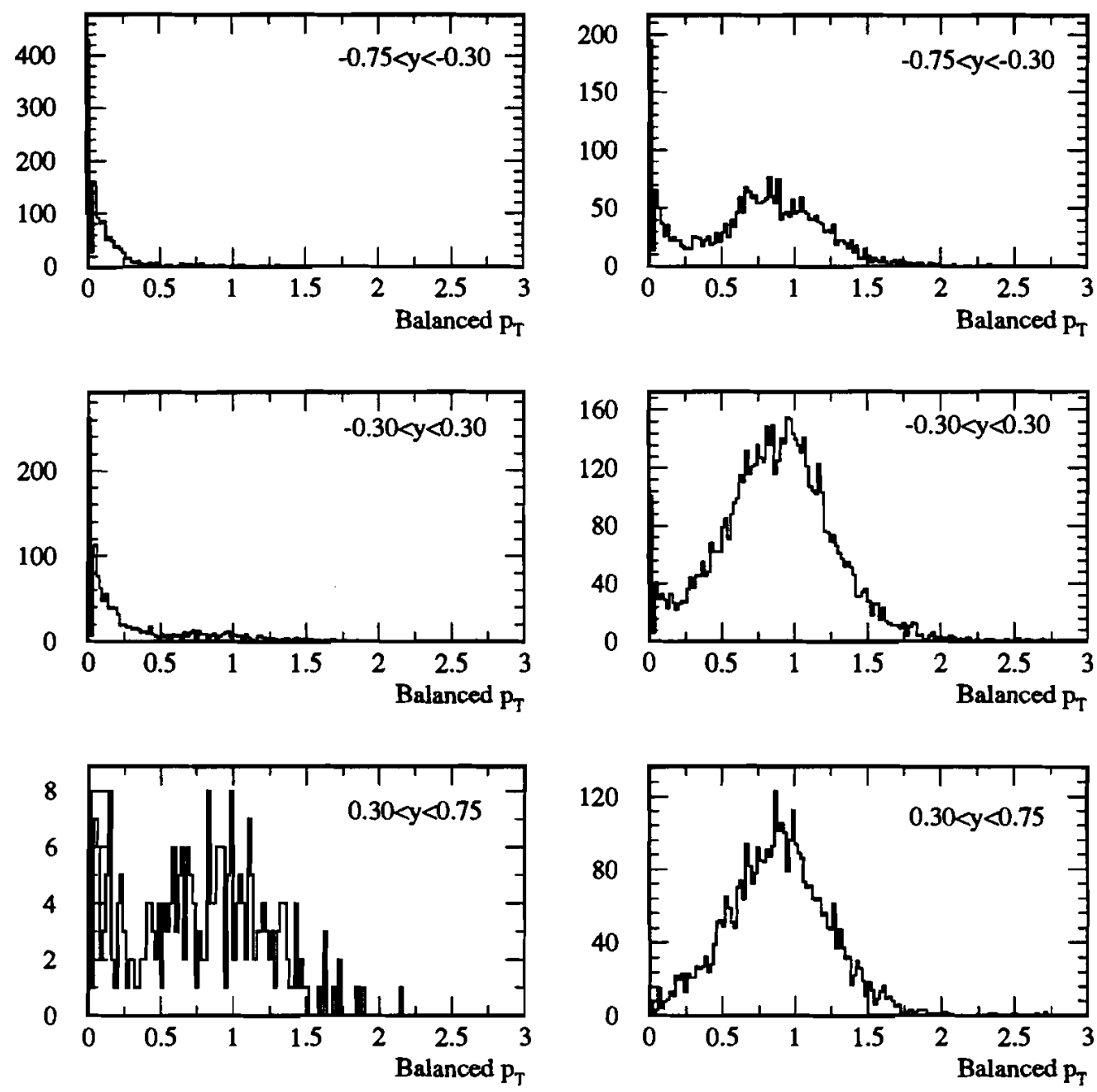

Figure 5.9: Balanced $p_{T}$ distributions for $\pi^{0}$ with $5.5<p_{T}<9.0 \mathrm{GeV} / \mathrm{c}$. Left column: events with off-line veto wall signal. Right column: Events without offline veto wall signal. 

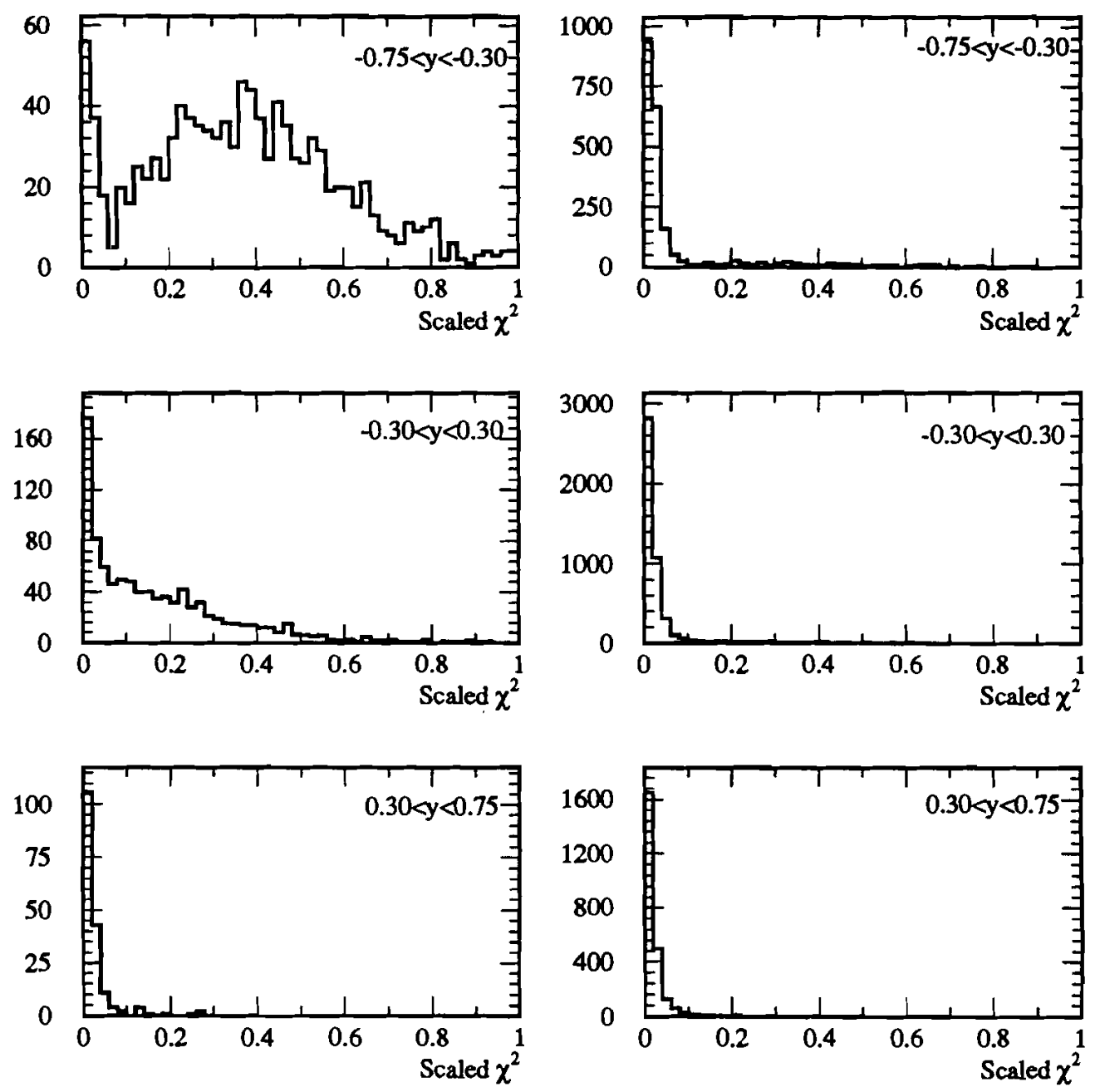

Figure 5.10: Photon scaled $\chi^{2}$ distribution for $\pi^{0}$ with $5.5<p_{T}<9.0 \mathrm{GeV} / \mathrm{c}$. Left column: events with off-line veto wall signal. Right column: Events without off-line veto wall signal. 
to the higher $p_{T}$ photon of the two forming the $\pi^{0}$. Figure 5.8 presents directionality spectra of that photon in three rapidity regions with the veto wall signal on and off. It can be seen that the number of photons characterised by positive directionality is larger in the backward than in the forward rapidity region. This is in agreement with the plots in Figure 5.7. The veto wall cut removed the majority of the muon like showers and the directionality cut set to 0.4 was applied to reject some of the remaining ones.

In events which trigger on high $p_{T}$ particles originating in the target, the transverse momentum on the trigger side of the calorimeter should be balanced by the total $p_{T}$ of the away side particles. In events containing showers originating from the beam halo, on other hand, we should observe $p_{T}$ imbalance. A measure of this effect, balanced $p_{T}$, was defined as the ratio of the $p_{T}$ of the away side jet to the $p_{T}$ of the $\pi^{0}$ candidate. The away side sum included charged tracks and neutral particle showers contained within a $240^{\circ}$ angle bisected by the continuation of the line drawn from the $\pi^{0}$ position through the center of the EMLAC to the away side. Figure 5.9 presents the balanced $p_{T}$ spectra in three rapidity regions for the high $p_{T} \pi^{0} \mathrm{~s}$. Events contributing to the sharp peak near zero contain muon like showers. The veto wall cut removed most of them and left a broad distribution centered around unity. Events with the balanced $p_{T}$ smaller than 0.1 were removed by the balanced $p_{T}$ cut.

Another variable designed to cut muon like showers was defined as the ratio of the photon $r$ view $\chi^{2}$ and its energy. The cut value was set to 0.1 and the cut was applied to the higher $p_{T}$ photon of the $\pi^{0}$ photon pair. Figure 5.10 presents the spectra of the scaled $\chi^{2}$ variable in three rapidity ranges. 


\subsubsection{The $\pi^{0}$ Signal Definition}

Figure 5.11 presents the invariant mass spectra of the $\pi^{0}$ and $\eta$ mesons after the above described cuts on a linear scale.

Gaussian fits gave a $\pi^{0}$ mass equal to $135 \mathrm{MeV} / \mathrm{c}^{2}$ and a peak $\mathrm{rms}$ of $6 \mathrm{MeV} / \mathrm{c}^{2}$, while for the $\eta$ they were $548 \mathrm{MeV} / \mathrm{c}^{2}$ and $21 \mathrm{MeV} / \mathrm{c}^{2}$. The $\pi^{0}$ signal used in the evaluation of the cross section was determined by subtracting twice the number of entries found in the two control regions of the mass peak (half sidebands) from the number of entries in the $\pi^{0}$ mass region $0.1<m<0.18 \mathrm{MeV} / \mathrm{c}^{2}$. For $\pi^{0}$ 's with $p_{T}$ smaller than $7.0 \mathrm{GeV} / \mathrm{c}$ sidebands were defined by the following conditions $0.08<m<0.10 \mathrm{MeV} / \mathrm{c}^{2}$ and $0.19<m<0.21 \mathrm{MeV} / \mathrm{c}^{2}$. For $p_{T}$ larger than 7.0 $\mathrm{GeV} / \mathrm{c}$, the respective ranges were: $0.09<m<0.10 \mathrm{MeV} / \mathrm{c}^{2}$ and $0.19<m<0.22$ $\mathrm{MeV} / \mathrm{c}^{2}$.

The mass spectra shown in Figure 5.11 are averaged over octants and available phase space. Figure 5.12 shows the dependence of the $\pi^{0}$ and $\eta$ mass on octant number, particle $p_{T}$ and meson radial position. The EMLAC energy scale was determined using $\eta$ and low $p_{T} \pi^{0}$ mesons, as their photons had significant separation. The energy scale was controlled with $0.5 \%$ accuracy.

Photons from $\pi^{0}$ decays can convert into $e^{+} e^{-}$pairs in the material downstream of the decay vertices. Since only two photon combinations were used in the physics analysis, any conversions happening upstream of the analysis magnet resulted in the loss of the $\pi^{0}$ candidates. To correct for this effect, for each $\pi^{0}$ candidate a probability of either photon converting was calculated based on the amount of the material present between the decay vertex and the magnet. 


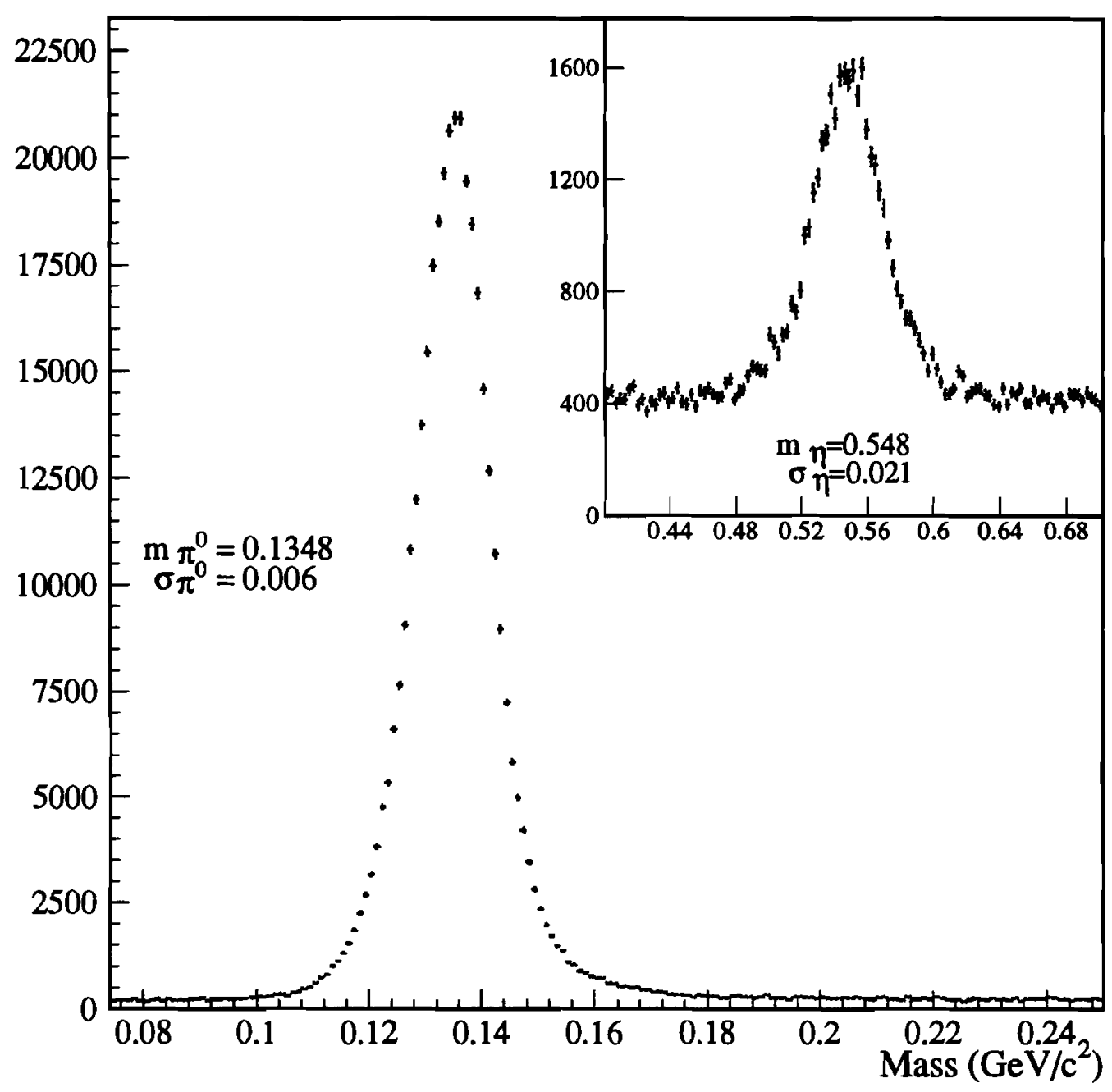

Figure 5.11: $\pi^{0}$ and $\eta$ mass distributions after the analysis cuts. 

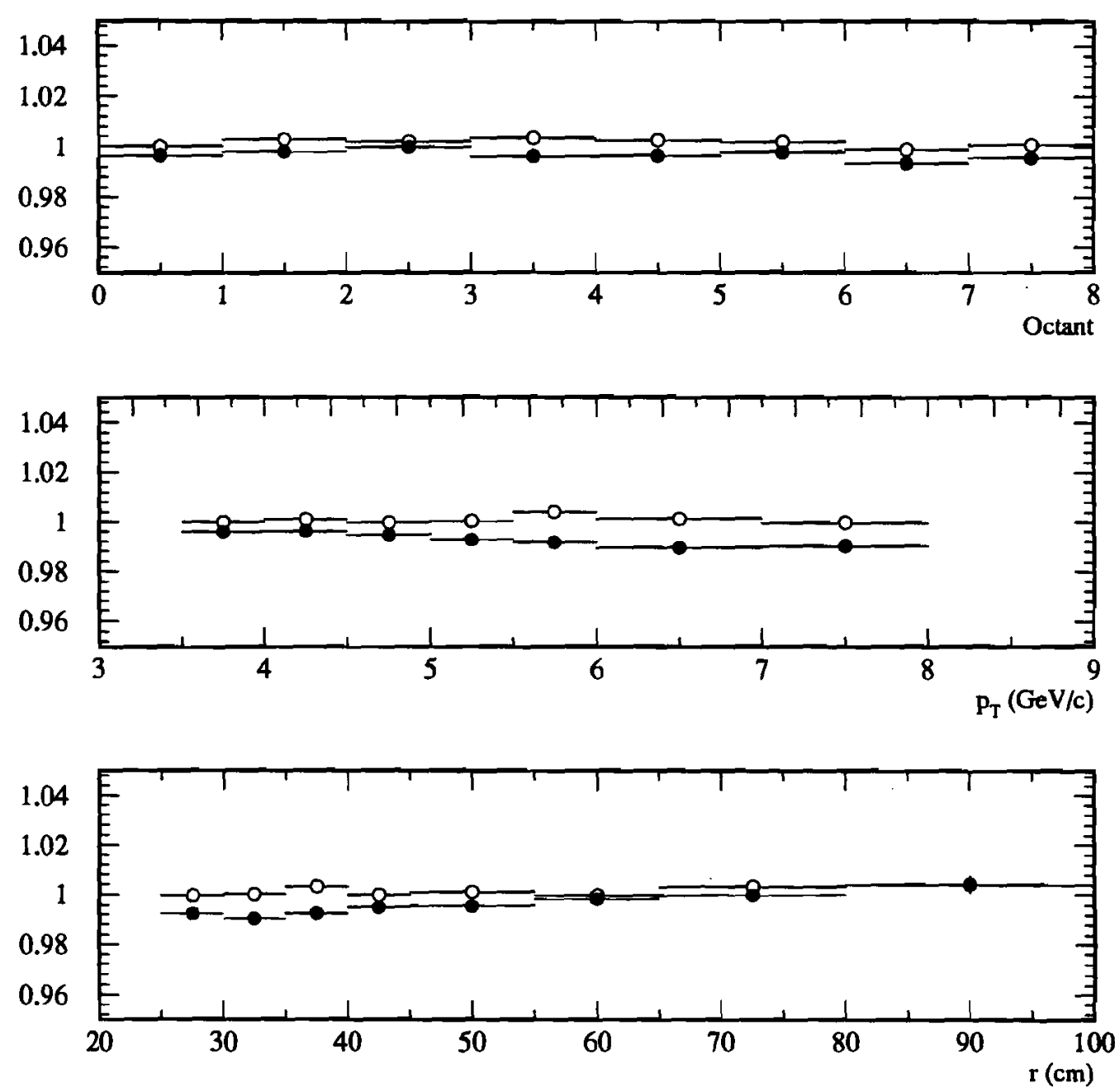

Figure 5.12: $\pi^{0}(\bullet)$ and $\eta(0)$ masses as functions of the octant number, $p_{T}$, and radial position. Masses are normalized to the world averages. 


\subsection{The Monte Carlo Simulation}

In order to determine the reconstruction efficiency for the $\pi^{0}$, as well as other particles, and to be able to estimate the background to the direct photon signal, E706 developed a sophisticated Monte Carlo (MC) simulation program. The steeply falling $p_{T}$ spectra of mesons and photons require that the simulation program be as faithful a representation of the detector and data taking process as possible.

Some simplifications were necessary, mainly in order to increase program efficiency. The multilayer LAC insulation was replaced by a homogenized material. A similar method was used to represent the copper clad readout boards. The thermal contraction of the EMLAC material was neglected. At this stage, the calorimeter was ideal and all channels were assumed to be operational and instrumented.

The E706 MC simulation program used HERWIG [64] as an event generator. It was superior to PYTHIA [65] in reproducing observed photon and charged tracks multiplicities [57]. Generated events were scanned for the presence of high $p_{T}$ particles such as $\pi^{0}, \omega$ or photon, which could produce EMLAC triggers. Events chosen this way were propagated through the GEANT [66] representation of the E706 apparatus assuming perfect readout electronics. Each shower that exceeded a certain energy cutoff was fully developed in the EMLAC down to the $10 \mathrm{MeV}$ threshold. Energies deposited in strips as well as remaining $10 \mathrm{MeV}$ shower components were stored in the data banks. The final shower development, below the $10 \mathrm{MeV}$ level, was done outside GEANT. A special purpose code was written to simplify the final stage of the shower propagation in order to decrease the simulation time per event [57].

Two major samples of MC events were generated, one rich in high $p_{T} \pi^{0}$ mesons and a second rich in $\eta$ mesons. Each sample was generated in sets with preset thresholds on the minimal $p_{T}$ of the generated mesons. This provided adequate 

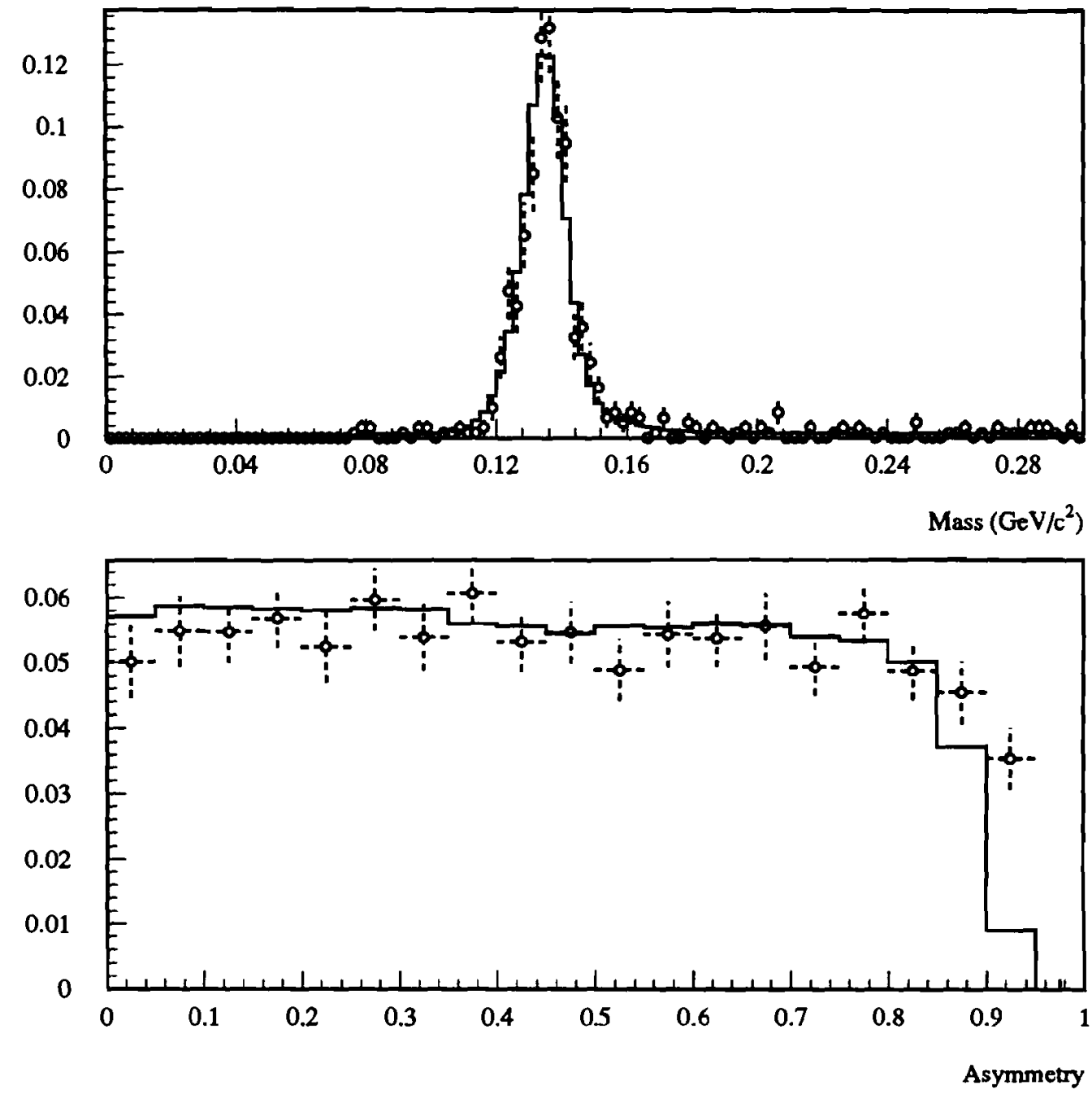

Figure 5.13: Top: $\pi^{0}$ candidates mass distributions from data (histogram) and Monte Carlo simulation ( 0 ). Bottom: $\pi^{0}$ candidates energy asymmetry distributions from data (histogram) and Monte Carlo simulation (o). Histograms are area normalized. 

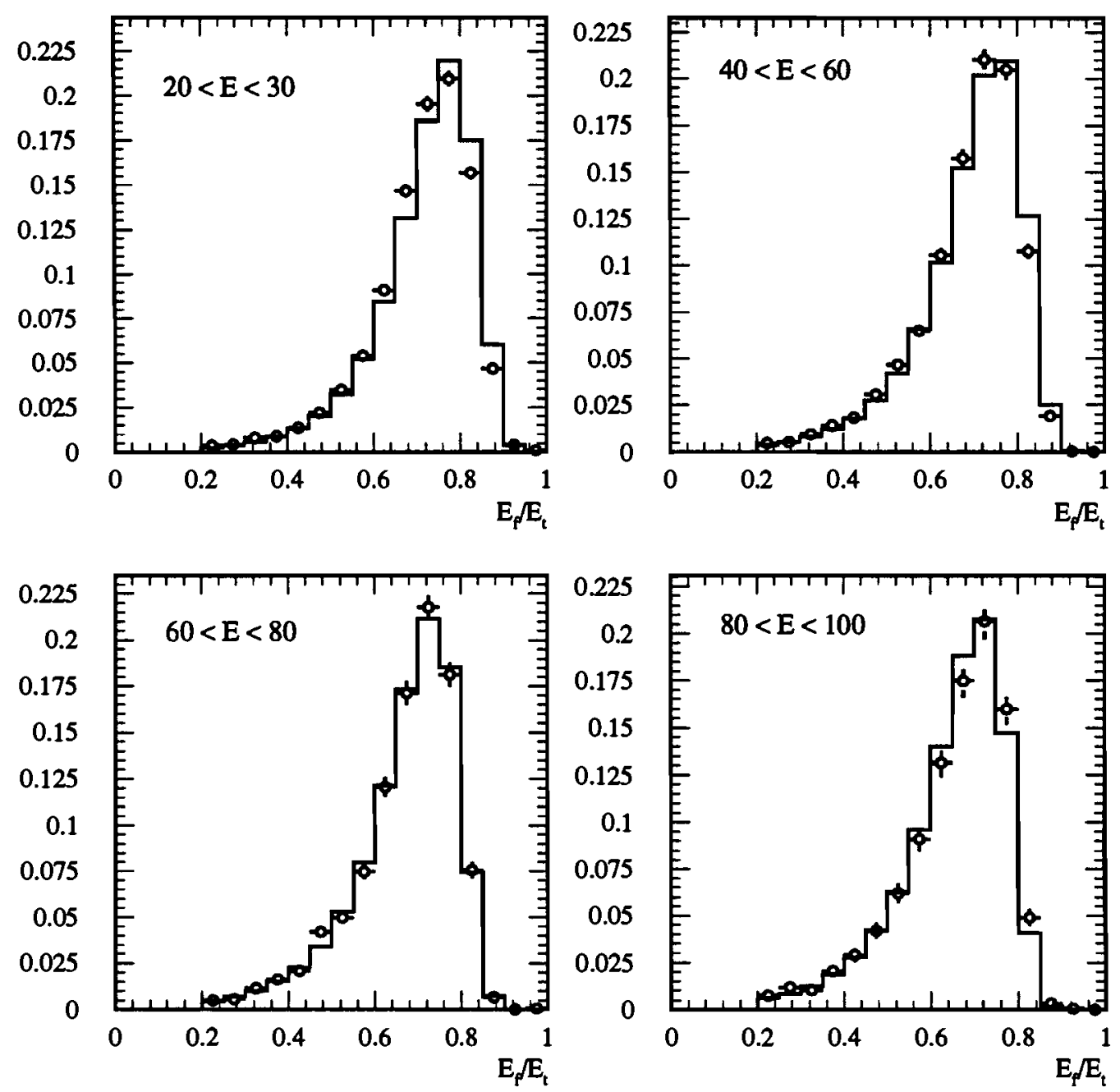

Figure 5.14: Photon $E_{f} / E_{t}$ distributions from the data (histogram) and the Monte Carlo simulation $(0)$ in selected photon energy bins. Histograms are area normalized. 
coverage of the full $p_{T}$ range without excessive statistics at the low $p_{T}$ end. MC DSTs were produced to match the running conditions of the runs used in the data analysis. Specifically, detector characteristics (locations of the dead channels, gain values, trigger thresholds) as stored in the run constants were applied to the ideal MC events. MC data were reconstructed using MAGIC and the energy scale of the $\mathrm{MC}$ was determined.

To ensure that MC events resemble data and can be used to determine reconstruction efficiencies accurately, a series of comparisons between simulation and data results were made. Fig. 5.13 shows the mass and asymmetry spectra for the $\pi^{0} \mathrm{~s}$. The agreement between the two curves is good, except the high asymmetry region. The background subtraction scheme described in the previous section was used in both cases. Figure 5.14 shows the comparison of the $E_{f} / E_{t}$ spectra of the data and Monte Carlo $\pi^{0}$ photons. This variable characterizes the longitudinal shower shape. The agreement between the two spectra is adequate. The MC events were used to determine several cut corrections.

\subsubsection{Reconstruction Efficiency}

MC events were used to calculate the reconstruction efficiency for $\pi^{0}$ mesons. The efficiency was defined as the ratio of the number of $\pi^{0} \mathrm{~s}$ reconstructed in a $p_{T}-y$ bin to the numbers of $\pi^{0} \mathrm{~s}$ generated inside the same bin. The reconstruction efficiency was evaluated using the $\mathrm{MC}$ with generation thresholds above $3 \mathrm{GeV}$. For each set, an offset of $0.5 \mathrm{GeV}$ from the generation threshold was used to properly account for the effects due to the energy resolution of the detector (slewing). Generated $\pi^{0} \mathrm{~s}$ had to emerge from the primary vertex inside the target fiducial region and decay into 2 photons. Photons could not convert in the material upstream from the magnet and had to hit the fiducial region of the same EMLAC octant. Only decays with asymmetries smaller than the cut value were accepted. The standard 
$\pi^{0}$ definition was used to count the number of reconstructed mesons with the exception of some muon rejection cuts and the $E_{f} / E_{t}$ cut; reconstruction efficiency was supposed to correct for the latter. Since all the physics analysis was performed in reconstructed variables, reconstruction efficiency was also used to correct for the difference between reconstructed and generated variables. A two dimensional surface fitted to the obtained distribution is shown in Figure 5.15. The following function was obtained from the fit:

$$
P_{\text {rec }}=0.973-e^{-3.609-5.01 \times y+0.965 \times p_{T}}
$$

The fitted range was $-0.75<y<0.75$ and $3.5<p_{T}<9.5$.

\subsection{Trigger Corrections}

The SLH trigger was based on the energy deposited in sixteen adjacent EMLAC r strips. There were 32 such overlapping groups in each octant. For each group, a trigger turnon curve was found, representing the efficiency of the trigger as a function of $p_{T}[49,67]$. Figure 5.16 shows sample curves of some sections for SLH and SINGLE LOCAL LOW (SLL) triggers. PRETRIGGER curves are shown in Figure 5.17. It can be seen that the SLH threshold was around $3.5 \mathrm{GeV}$ and the trigger was fully efficient around $4.5 \mathrm{GeV}$. For each event containing a SLH trigger, the probability that the trigger fires was calculated. The calculation was based upon the trigger turnon curves and energies deposited in groups of 8 strips. The formula for the trigger probability, $P_{S L H}$, was:

$$
P_{S L H}=1-\prod_{\text {sums }- \text { of }-8}\left(1-p_{i}\right)
$$

where $p_{i}$ was the probability of the $\mathrm{i}$-th group trigger firing in a given octant. Each $\pi^{0}$ candidate was then weighted by the inverse of $P_{S L H}$. Events with large weights, or $P_{S L H}<.05$, were cut. This cut was also implemented in the MC simulation. 


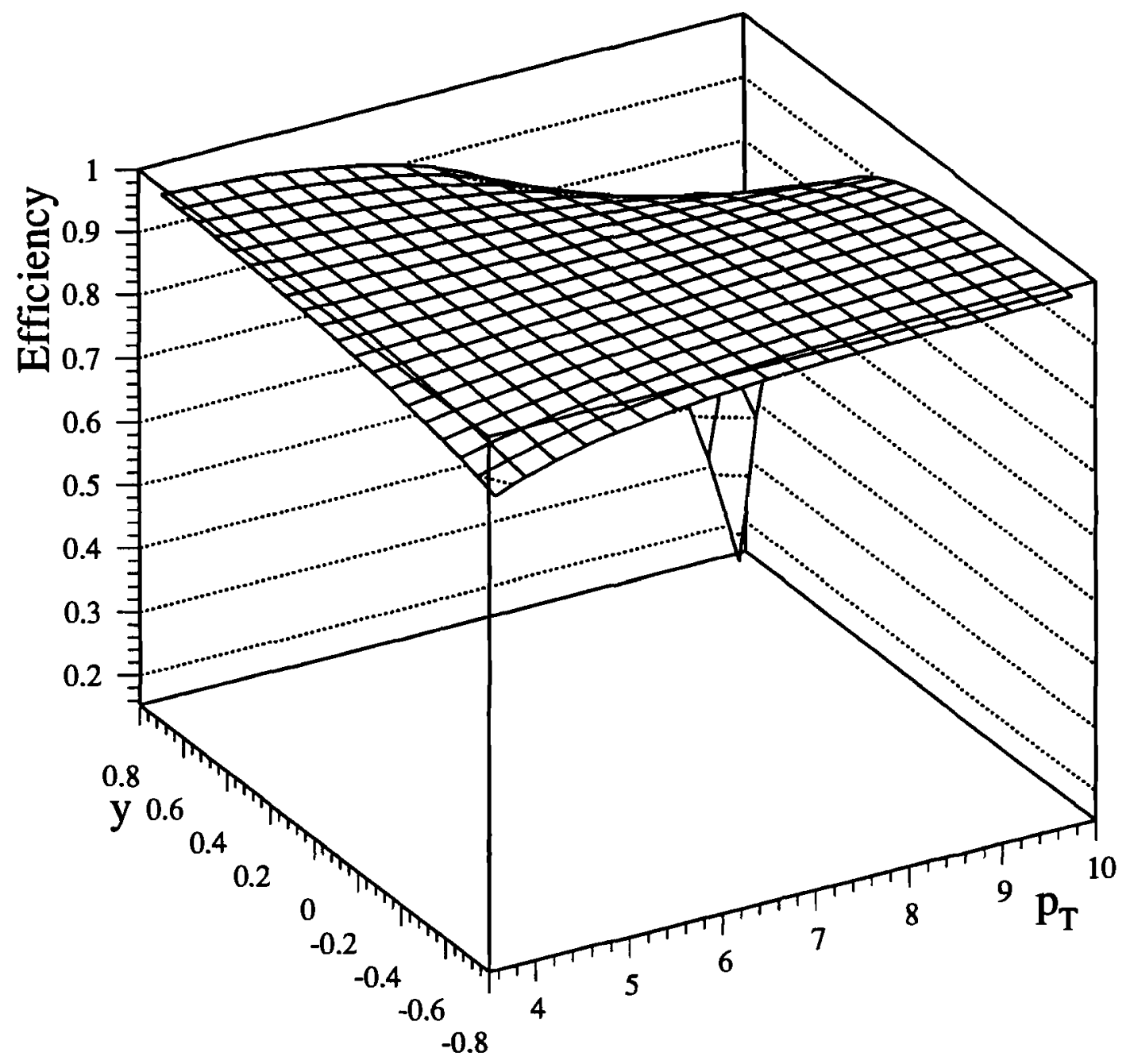

Figure 5.15: The $\pi^{0}$ reconstruction efficiency as a function of $p_{T}$ and $\mathrm{y}$. 

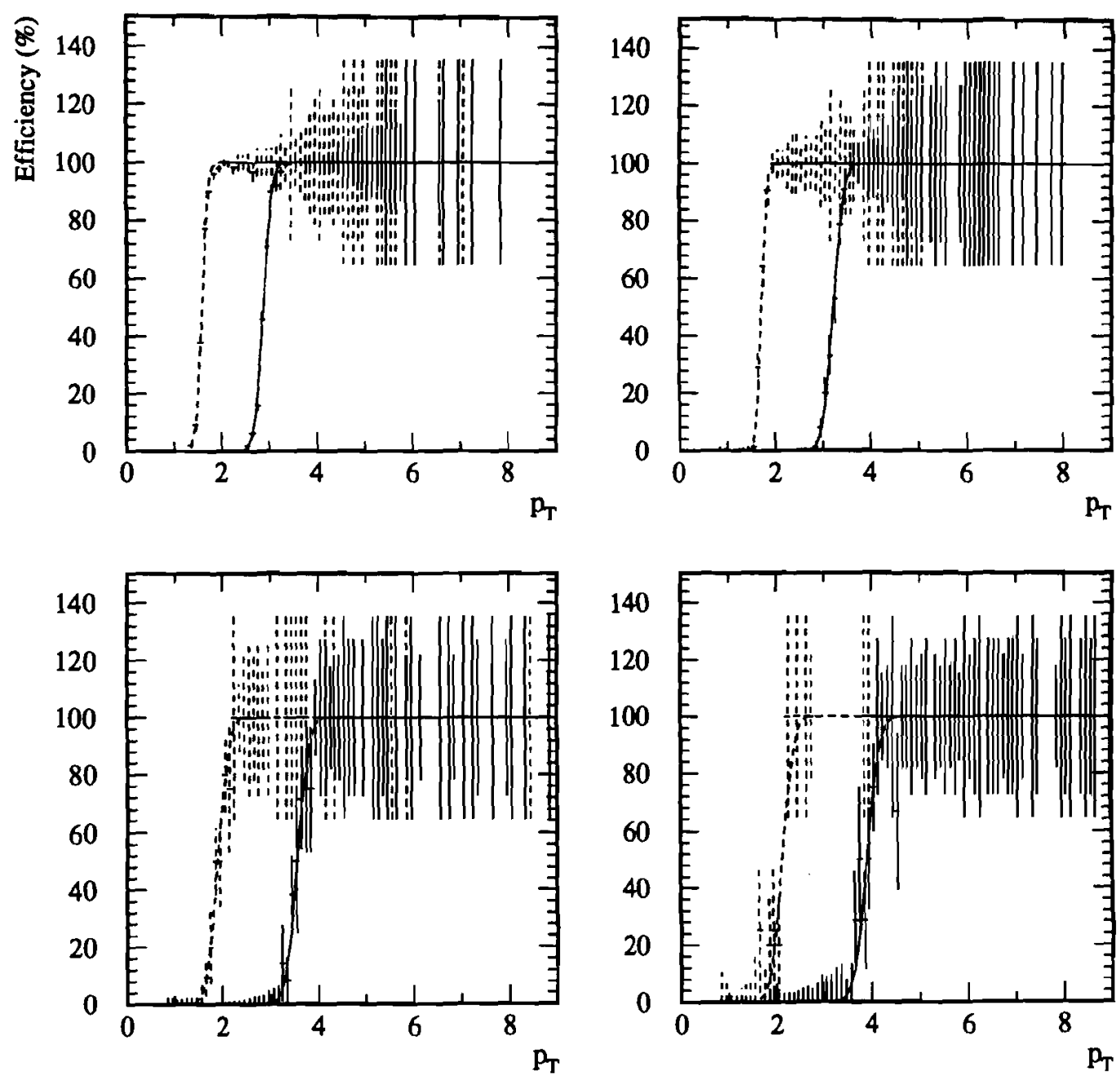

Figure 5.16: Trigger efficiency curves for SLH (solid line) and SLL (dashed line) triggers for selected sections in the inner (top) and outer (bottom) sections of an octant as functions of the trigger $p_{T}$. 

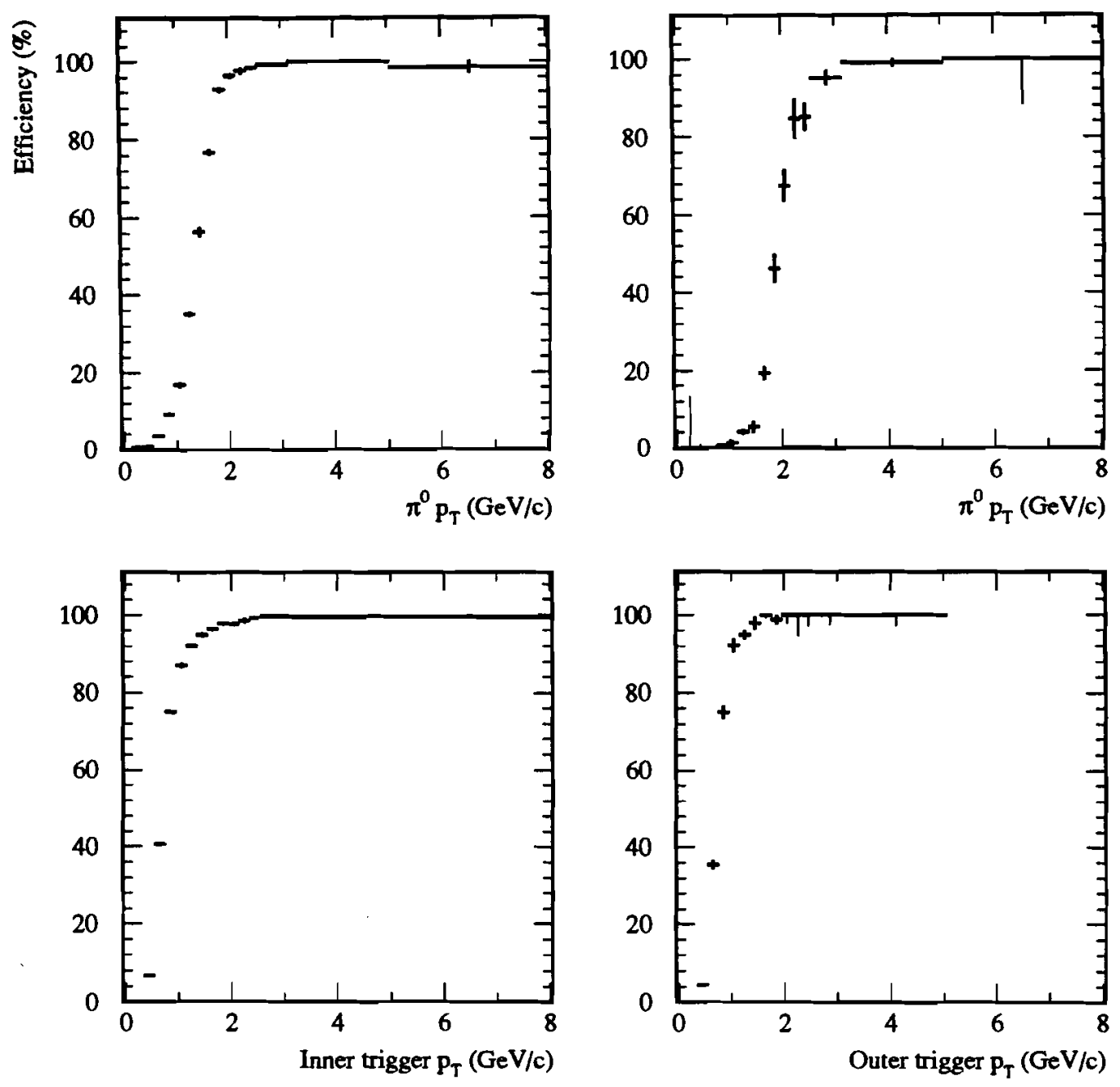

Figure 5.17: PRETRIGGER efficiency curves for the inner (left column) and outer (right column) sections of an octant as functions of the trigger and $\pi^{0} p_{T}$. 


\subsection{Beam Normalization and Energy}

During event readout, the DA system was disabled from accepting triggers. To evaluate the beam normalization several scalers were used in the calculation of the Live Triggerable Beam (LTB) factor [68] defined as:

$$
N_{L T B}=N_{B E A M 1 \cdot \overline{B E}} \bullet L I V E_{-} F R A C T I O N
$$

$N_{B E A M 10 \overline{B H}}$ was the number of beam particles for which the condition $B E A M 1$ • $\overline{B H}$ was TRUE; $B E A M 1$ and $\overline{B H}$ were defined in Chapter 3. LIVE_FRACTION determined the fraction of beam particles which could produce an acceptable trigger. It was defined as a product of the following terms:

- Clean Interaction Fraction - the fraction of interactions without another interaction within $\pm 60 \mathrm{~ns}$ of the interaction of interest.

- Computer Live Fraction - the fraction of time the DA was ready to accept a new trigger.

- Pretrigger Live Fraction and Trigger Live Fraction - this accounted for the time when the on-line veto wall veto, early $p_{T}$ veto, and the power supplies noise spike vetoing were not active.

The $\pi^{-}$beam momentum was determined as $515 \mathrm{GeV} / \mathrm{c}$ by calibrating magnet settings using the primary $800 \mathrm{GeV} / \mathrm{c}$ beam. The RMS of the momentum distribution was $30 \mathrm{GeV} / \mathrm{c}$. This measurement was confirmed using the E706 tracking system [69]. A sample of tracks was selected from prescaled BEAM trigger events. Events of interest did not have reconstructed vertices and had good SSD and PWC track. The well defined impulse delivered by the analysis magnet (calibrated from upstream $K_{s}$ and $J / \psi$ decays) enabled this estimation of the beam energy. 


\subsection{The Cross Section Definition}

The invariant cross section per nucleon ${ }^{4}$ was defined as:

$$
E \frac{d^{3} \sigma}{d p^{3}}=\frac{1}{2 \pi p_{T} \Delta p_{T} \Delta y} \frac{1}{\rho l N_{A}} \frac{N_{\pi^{0}}^{\text {corr }}}{N_{L T B}} C
$$

The first fraction on the right represents the phase space term in $p_{T}$ and $y$ variables, the second is the target nucleon count ( $\rho$ is the target density, $l$ its length and $N_{A}$ Avogadro's number) and the third is a ratio of the corrected number of pions produced in a $p_{T}-y$ bin $N_{\pi^{0}}^{\text {corr }}$ and the Live Triggerable Beam factor $N_{L T B} . N_{\pi^{0}}^{\text {corr }}$ was defined as the sum of the products of the following event dependent terms (weights):

- $W_{\text {trigger }}$ - octant trigger weight. Inverse of $P_{S L H}$ from Equation 5.4.

- Wacceptance - inverse of the azimuthal acceptance of the EMLAC.

- $W_{\text {reconstruction }}$ - inverse of $P_{\text {rec }}$ from Equation 5.3. function.

- $W_{\text {conversions }}$ - inverse of the $\pi^{0}$ photons non-conversion probability.

- $W_{\text {beam absorption }}$ - inverse of the beam non-absorption probability.

The term $C$ represents the product of the following constant correction factors:

- $W_{\text {asymmetry }}$ - inverse of the energy asymmetry cut value.

- $W_{\text {veto wall }}$ - veto wall cut correction.

- $W_{\text {directionality }}$ - directionality cut correction.

- $W_{\text {balanced pT }}-$ balanced $p_{T}$ cut correction.

- $W_{\text {scaled } \chi^{2}}$ scaled $\chi^{2}$ cut correction.

\footnotetext{
${ }^{4}$ All resulting cross sections will be presented in $\mathrm{pb} /(\mathrm{GeV} / \mathrm{c})^{2}$.
} 
- $W_{\text {beam muons }}$ - correction for the beam muon contamination [70].

- $W_{\text {target }}-$ correction for the target fiducial volume cut.

- $W_{\text {vertex }}$ - inverse of the vertex reconstruction efficiency [54].

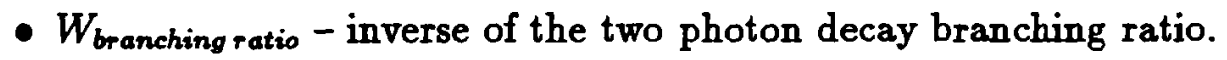

Table 5.2 presents averaged correction values applied to the data. 


\begin{tabular}{|c|c|}
\hline Correction Type & Averaged Correction \\
\hline \hline photon conversions & $1.175(\mathrm{Be})$ \\
beam absorption & $1.380(\mathrm{Cu})$ \\
asymmetry cut & $1.007(\mathrm{Cu})$ \\
veto wall cut & 1.333 \\
directionality cut & 1.05 \\
balanced $p_{T}$ cut & 1.021 \\
scaled $\chi^{2}$ cut & 1.053 \\
beam contamination & 1.016 \\
target fiducial region & 1.005 \\
vertex reconstruction & 1.35 \\
\hline
\end{tabular}

Table 5.2: Summary of the averaged corrections. 


\section{Chapter 6}

\section{Results}

\subsection{Cross Sections}

$\pi^{0}$ production cross sections were calculated as a functions of pion transverse momentum and rapidity. Figures 6.1 and 6.2 present the invariant cross section of inclusive $\pi^{0}$ production on $\mathrm{Be}$ and $\mathrm{Cu}$ target, respectively. The cross sections were calculated per nucleon over the c.m. rapidity range $-0.75<y<0.75$. Tables 6.1 and 6.2 contain the values of the cross sections together with their statistical errors. The power determining the rate of fall of the cross section as a function of $p_{T}$ can be found by fitting the plots presented in Figures 6.1 and 6.2 with the phenomenological formula (used for example in [27] ):

$$
E \frac{d^{3} \sigma}{d p^{3}}=C \frac{\left(1-x_{T}\right)^{m}}{p_{T}^{n}}
$$

where $x_{T}=2 p_{T} / \sqrt{s}$ and $C$ is a normalization constant.

Figures 6.3 and 6.4 present $\pi^{0}$ production cross sections on a Be target in seven rapidity bins. The overall shapes are similar. Rapidity profiles of the cross section are presented in Figure 6.5. The distributions are shifted towards positive rapidity values due to the (on average) larger energy of the beam partons, since in the c.m. system, beam momentum is shared among two valence quarks (and gluons), while the equal nucleon momentum is shared by three quarks (and gluons). 


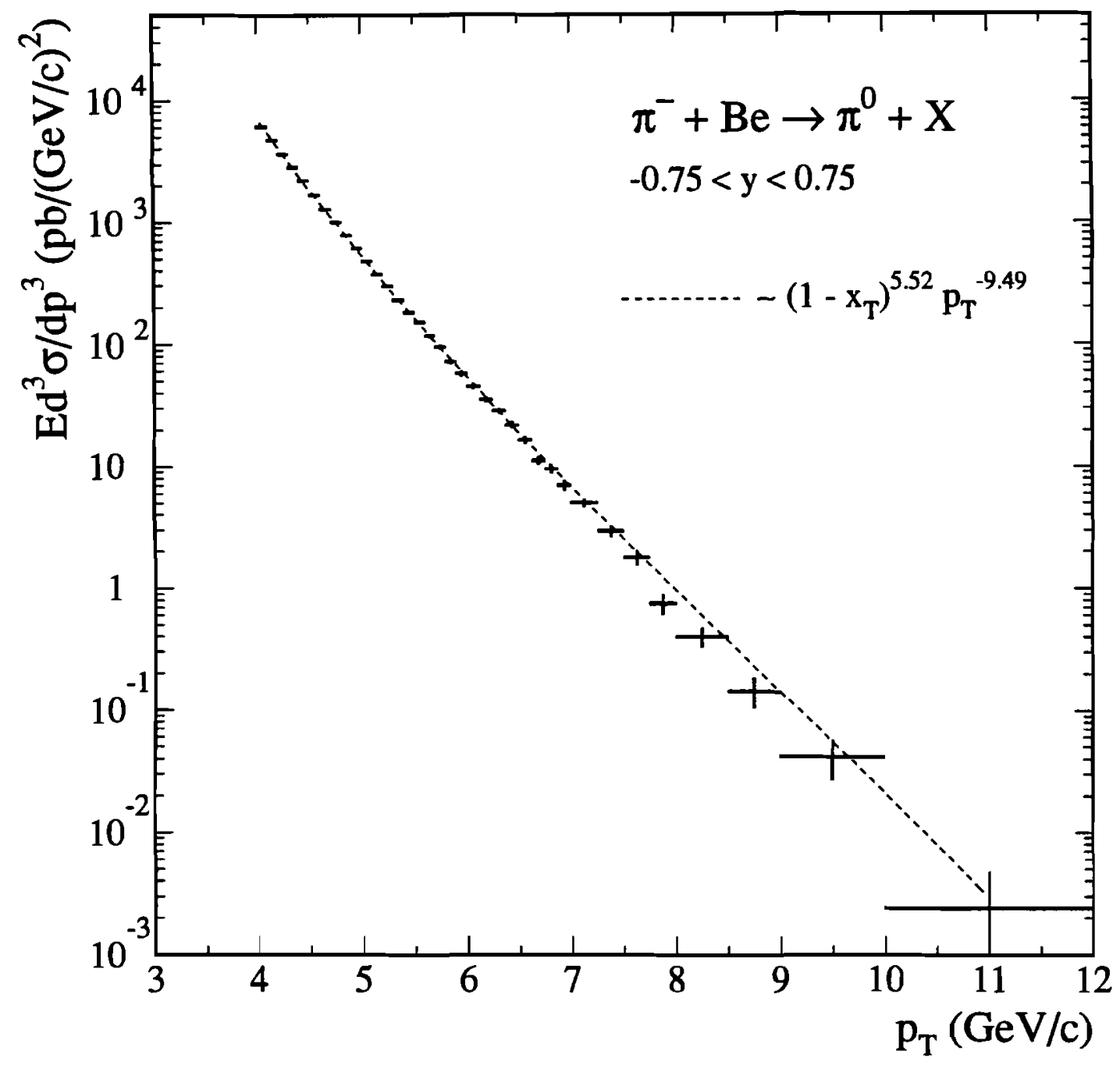

Figure 6.1: The $p_{T}$ dependence of the $\pi^{0}$ invariant production cross section per nucleon on a Be target. The dashed line is a fit to Equation 6.1. 


\begin{tabular}{||c|c|c|c||}
\hline \hline$p_{T}(\mathrm{GeV} / \mathrm{c})$ & $\begin{array}{c}\pi^{-}+\mathrm{Be} \rightarrow \pi^{0}+\mathrm{X} \\
-0.75<y<0.75\end{array}$ & $p_{T}(\mathrm{GeV} / \mathrm{c})$ & $\pi^{-}+\mathrm{Be} \rightarrow \pi^{0}+\mathrm{X}$ \\
& & & \\
\hline & & & \\
\hline $4.000-4.100$ & $6070 \pm 40$ & $5.800-5.900$ & $71.9 \pm 2.8$ \\
$4.100-4.200$ & $4710 \pm 30$ & $5.900-6.000$ & $57.1 \pm 2.3$ \\
$4.200-4.300$ & $3610 \pm 30$ & $6.000-6.125$ & $44.6 \pm 1.8$ \\
$4.300-4.400$ & $2830 \pm 20$ & $6.125-6.250$ & $35.2 \pm 1.6$ \\
$4.400-4.500$ & $2190 \pm 20$ & $6.250-6.375$ & $28.4 \pm 1.4$ \\
$4.500-4.600$ & $1660 \pm 20$ & $6.375-6.500$ & $21.8 \pm 1.3$ \\
$4.600-4.700$ & $1280 \pm 20$ & $6.500-6.625$ & $16.4 \pm 1.1$ \\
$4.700-4.800$ & $1000 \pm 13$ & $6.625-6.750$ & $11.1 \pm 0.9$ \\
$4.800-4.900$ & $776 \pm 11$ & $6.750-6.875$ & $9.51 \pm 0.74$ \\
$4.900-5.000$ & $606 \pm 9$ & $6.875-7.000$ & $6.96 \pm 0.69$ \\
$5.000-5.100$ & $478 \pm 8$ & $7.000-7.250$ & $5.00 \pm 0.40$ \\
$5.100-5.200$ & $374 \pm 7$ & $7.250-7.500$ & $2.94 \pm 0.31$ \\
$5.200-5.300$ & $297 \pm 6$ & $7.500-7.750$ & $1.79 \pm 0.23$ \\
$5.300-5.400$ & $229 \pm 5$ & $7.750-8.000$ & $0.75 \pm 0.14$ \\
$5.400-5.500$ & $182 \pm 5$ & $8.000-8.500$ & $0.40 \pm 0.08$ \\
$5.500-5.600$ & $150 \pm 4$ & $8.500-9.000$ & $0.14 \pm 0.04$ \\
$5.600-5.700$ & $117 \pm 4$ & $9.000-10.000$ & $0.04 \pm 0.02$ \\
$5.700-5.800$ & $95.4 \pm 3.1$ & $10.000-12.000$ & $0.002 \pm 0.002$ \\
& & & \\
\hline \hline
\end{tabular}

Table 6.1: The $\pi^{0}$ invariant cross section per nucleon on a Be target. 


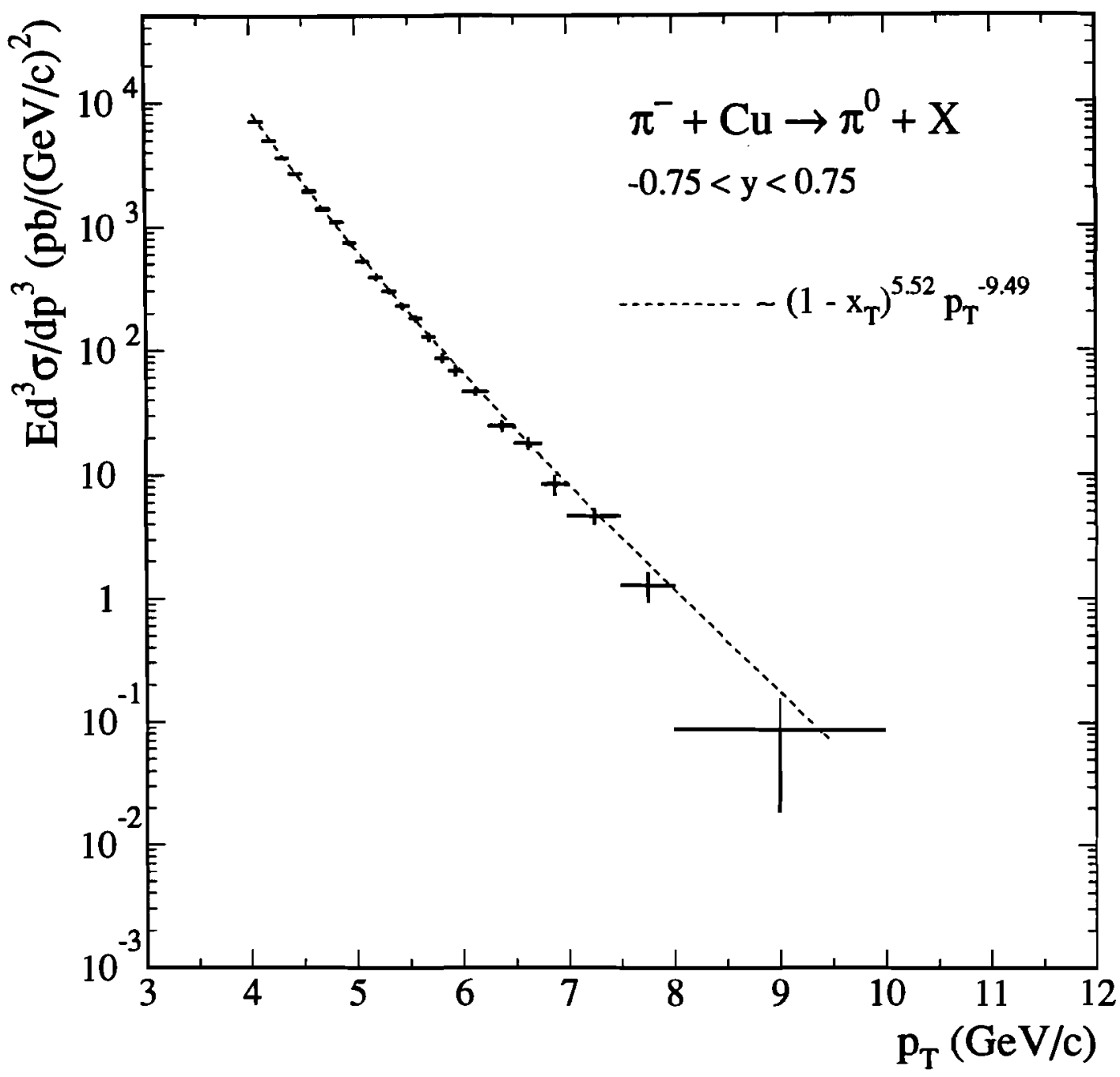

Figure 6.2: The $p_{T}$ dependence of the $\pi^{0}$ invariant production cross section per nucleon on a $\mathrm{Cu}$ target. The dashed line is a fit presented in Figure 6.1. 


\begin{tabular}{||c|c||}
\hline \hline$p_{T}(\mathrm{GeV} / \mathrm{c})$ & $\pi^{-}+\mathrm{Cu} \rightarrow \pi^{0}+\mathrm{X}$ \\
& $-0.75<y<0.75$ \\
& \\
\hline \hline $4.000-4.125$ & $7060 \pm 110$ \\
$4.125-4.250$ & $4940 \pm 90$ \\
$4.250-4.375$ & $3620 \pm 70$ \\
$4.375-4.500$ & $2670 \pm 60$ \\
$4.500-4.625$ & $1940 \pm 40$ \\
$4.625-4.750$ & $1380 \pm 40$ \\
$4.750-4.875$ & $1080 \pm 30$ \\
$4.875-5.000$ & $739 \pm 25$ \\
$5.000-5.125$ & $520 \pm 19$ \\
$5.125-5.250$ & $383 \pm 16$ \\
$5.250-5.375$ & $298 \pm 14$ \\
$5.375-5.500$ & $226 \pm 12$ \\
$5.500-5.625$ & $180 \pm 11$ \\
$5.625-5.750$ & $127 \pm 9.4$ \\
$5.750-5.875$ & $85.5 \pm 7.3$ \\
$5.875-6.000$ & $67.8 \pm 6.6$ \\
$6.000-6.250$ & $45.9 \pm 3.4$ \\
$6.250-6.500$ & $24.5 \pm 2.4$ \\
$6.500-6.750$ & $17.7 \pm 2.04$ \\
$6.750-7.000$ & $8.32 \pm 1.52$ \\
$7.000-7.500$ & $4.67 \pm 0.72$ \\
$7.500-8.000$ & $1.28 \pm 0.34$ \\
$8.000-10.000$ & $0.09 \pm 0.07$ \\
& \\
\hline \hline
\end{tabular}

Table 6.2: The $\pi^{0}$ invariant cross section per nucleon on a $\mathrm{Cu}$ target. 

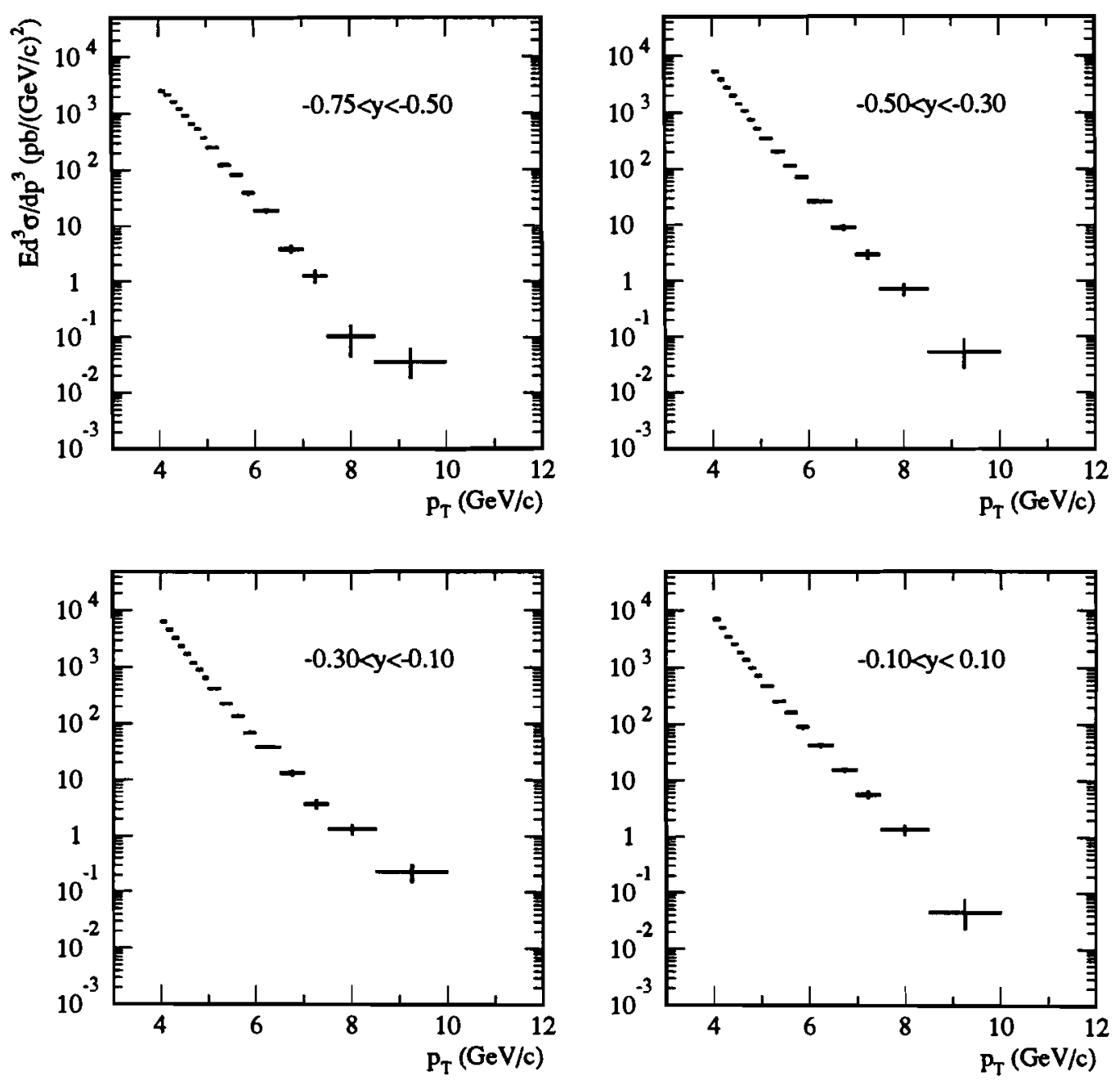

Figure 6.3: The $p_{T}$ dependence of the $\pi^{0}$ invariant production cross section per nucleon on a Be target in selected $(y \leq 0)$ rapidity bins. 

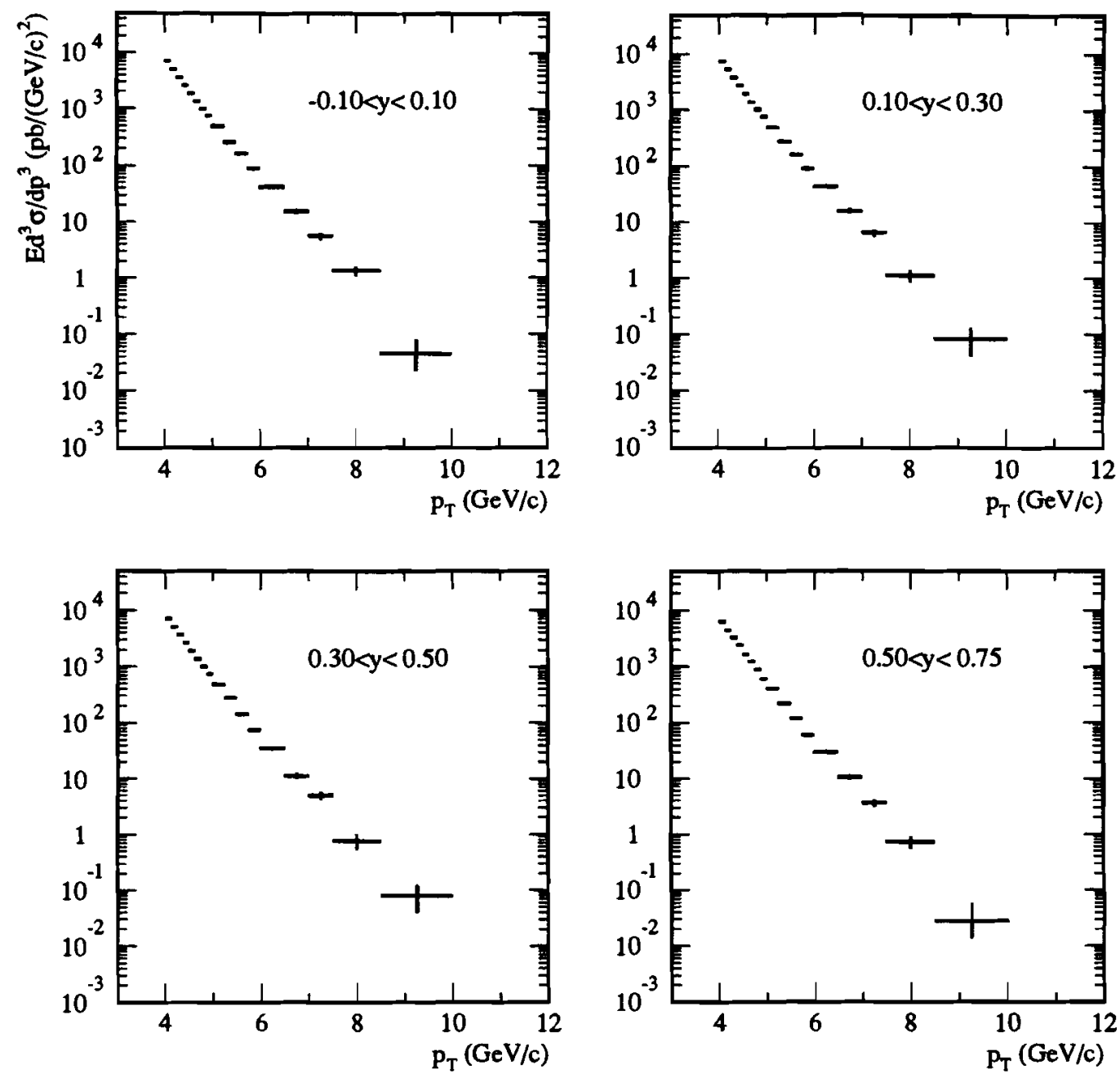

Figure 6.4: The $p_{T}$ dependence of the $\pi^{0}$ invariant production cross section per nucleon on a $\mathrm{Be}$ target in selected $(y \geq 0)$ rapidity bins. 

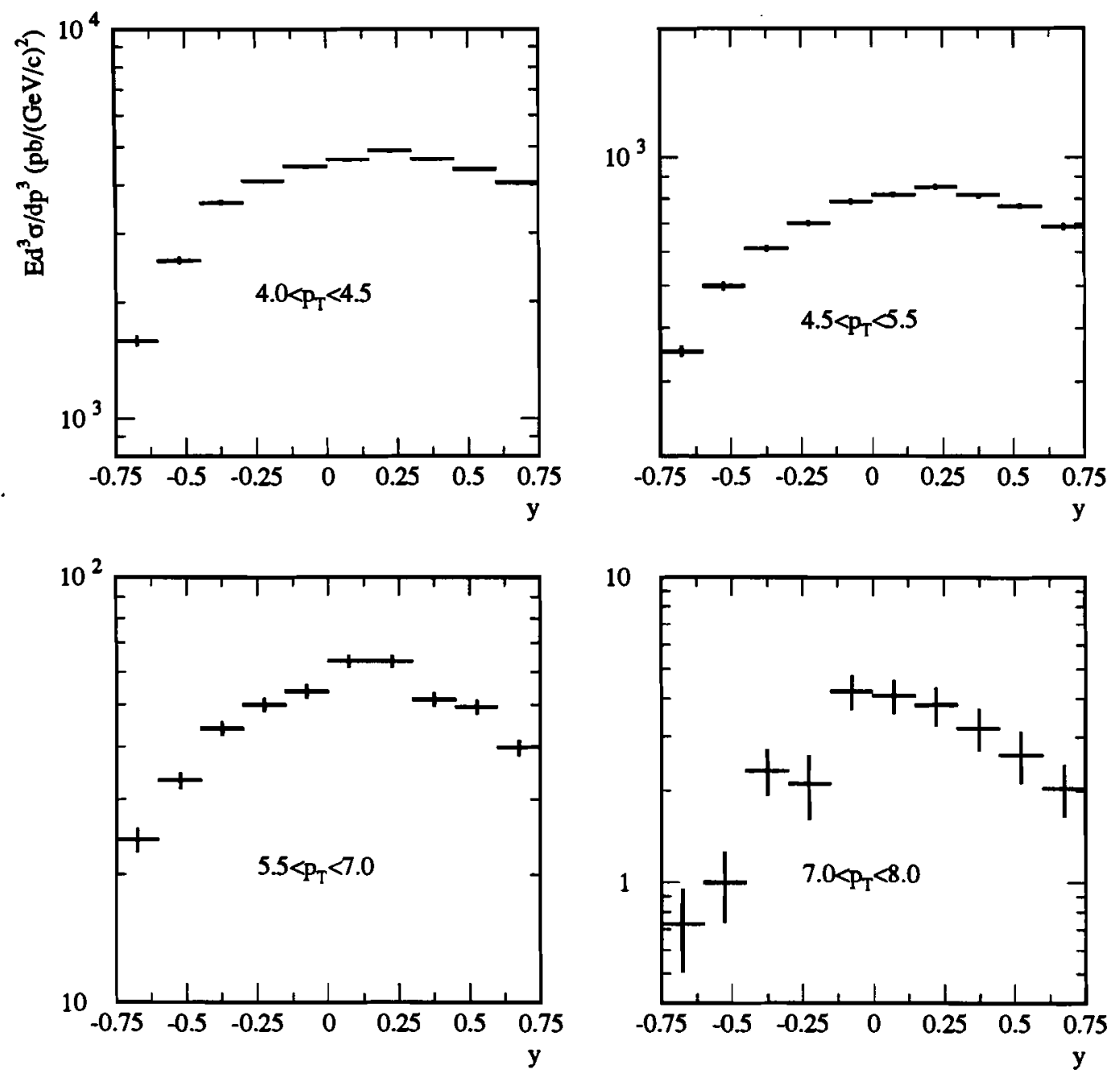

Figure 6.5: The rapidity dependence of the $\pi^{0}$ invariant production cross section per nucleon on a Be target in selected $p_{T}$ bins. 


\subsection{Comparisons with Other Experiments}

Figure 6.6 shows the E706 results on $\pi^{0}$ production cross section together with the results of the experiments mentioned in Chapter 1. The E706 cross section is higher than the ones from the other experiments because E706 used a higher energy pion beam. Considering the statistical significance of its data, and its much larger $p_{T}$ range ${ }^{1}$, E706 is clearly the definitive study of $\pi^{0}$ production by a $\pi^{-}$beam.

\subsection{Nuclear Dependence}

We use Equation 1.11 to determine the parameter $\alpha$ which describes the nuclear dependence of the $\pi^{0}$ production cross section.

Figure 6.7 presents the $p_{T}$ and $y$ dependence of $\alpha$. The $p_{T}$ dependence is averaged over the rapidity range $-0.75<y<0.75$. The values of $\alpha$ are listed in Table 6.3. In the region $4.0<p_{T}<10.0, \alpha$ is constant and its value is found to be 1.085 . Figure 6.8 also presents the rapidity dependence of $\alpha$ in several $p_{T}$ bins; we see that $\alpha$ is nearly independent of rapidity over the range studied.

In Figure 6.8 E706 values of $\alpha$ are compared to values obtained by E258 for charged pion production in the central rapidity region. The nuclear effects on the production of high $p_{T} \pi^{0}$ 's by a $515 \mathrm{GeV} / \mathrm{c} \pi^{-}$beam is consistent with that observed in the production of high $p_{T}$ charged pions by a $200 \mathrm{GeV} / \mathrm{c} \pi^{-}$beam.

\subsection{Comparison with Theoretical Predictions}

As mentioned in Chapter 1 , full NLL calculations for $\pi^{0}$ production are available [19]. The code uses ABFKW parton distribution functions for pions [21], ABFOW for nucleons [22] and the pion fragmentation function of [23]. The distri-

\footnotetext{
${ }^{1}$ The analysis of the data from the SLL, GLOBAL and INTERACTION triggers will extend the $p_{T}$ range of $\mathrm{E} 706$ down to $0.5 \mathrm{GeV} / \mathrm{c}$.
} 


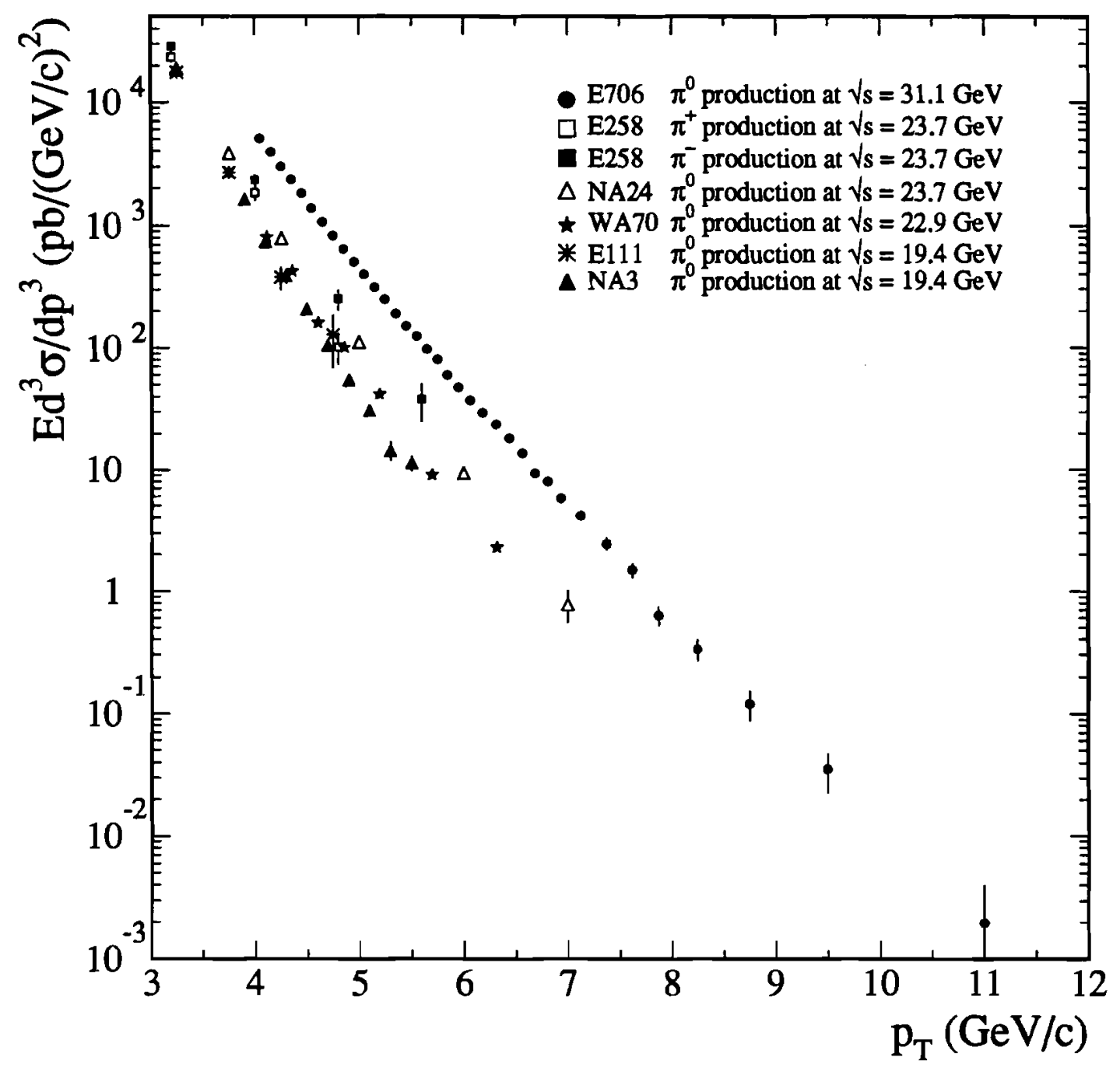

Figure 6.6: Comparison of the E706 $\pi^{0}$ invariant cross section on a Be target to the results from other experiments. Results of the experiments E706 and NA3 have been corrected for nuclear dependence (see Section 6.3). 

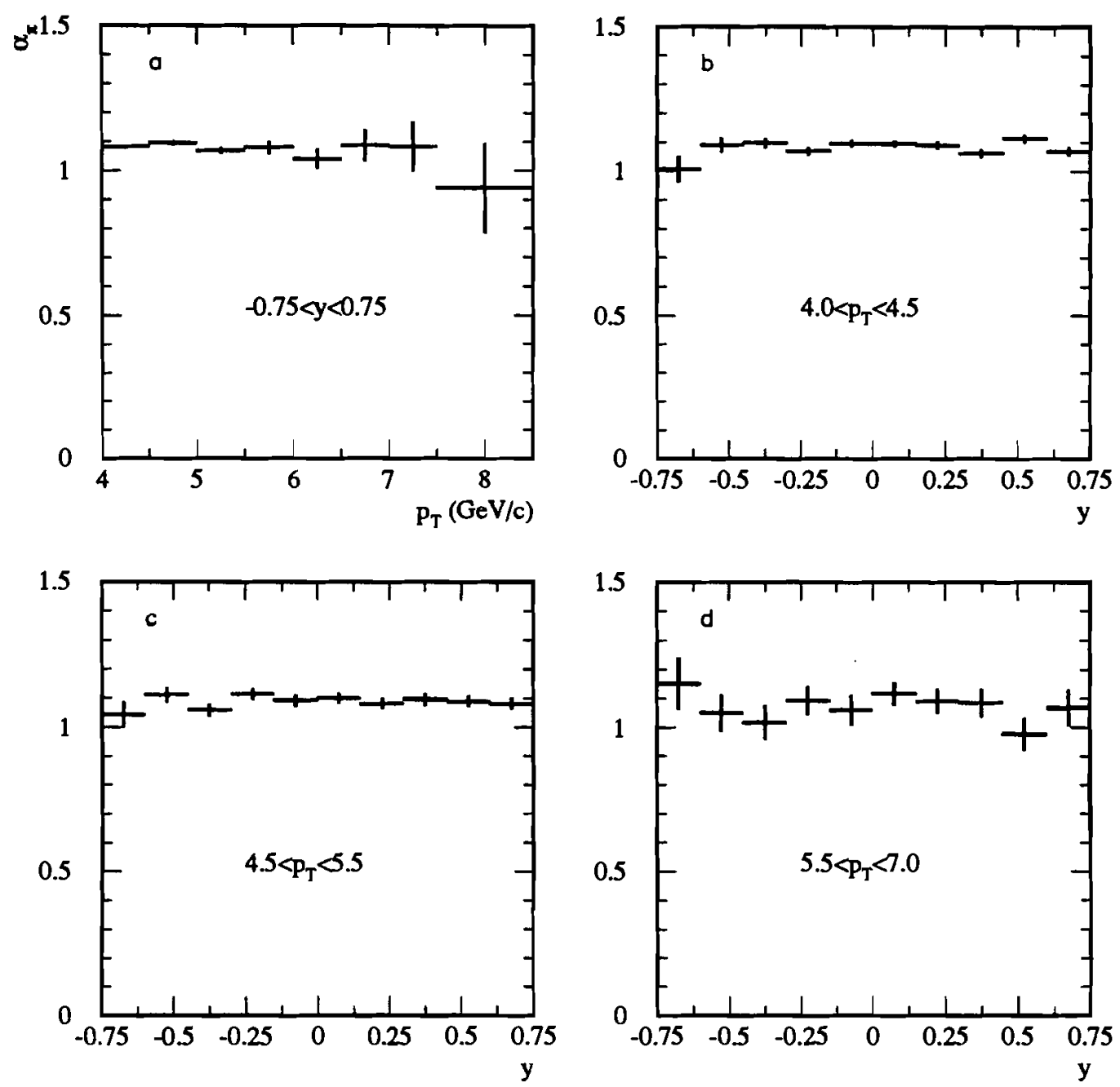

Figure 6.7: a) The $p_{T}$ dependence of $\alpha$. b), c), d) The rapidity dependence of $\alpha$ in selected $p_{T}$ bins. 


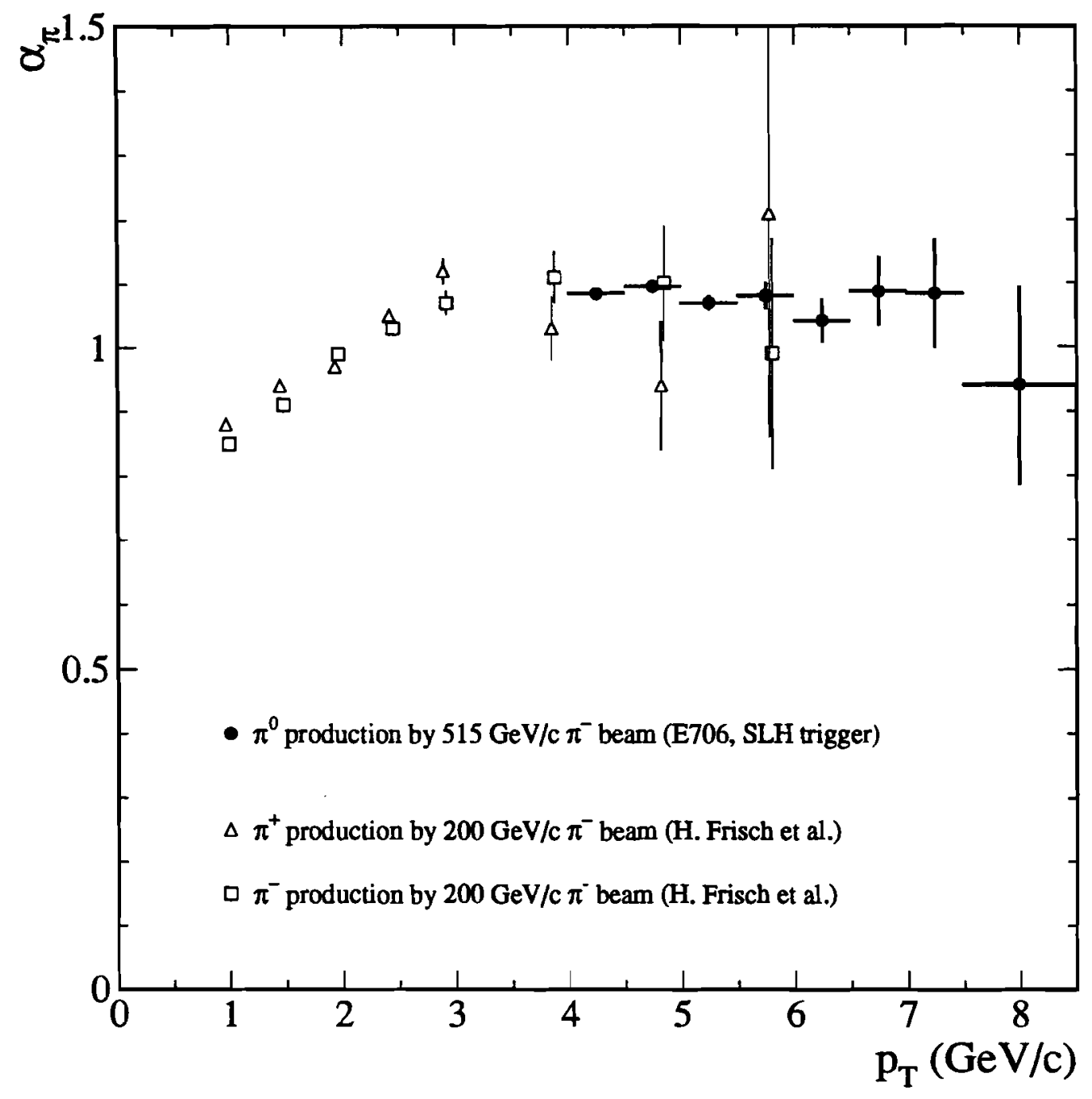

Figure 6.8: The $p_{T}$ dependence of $\alpha$ for $\pi^{-}, \pi^{+}$and $\pi^{0}$ production. Charged pion data are from Reference 26. 


\begin{tabular}{|c|c|}
\hline \hline$p_{T}(\mathrm{GeV} / \mathrm{c})$ & $\alpha$ \\
\hline \hline & \\
\hline $4.0-4.5$ & $1.084 \pm 0.005$ \\
$4.5-5.0$ & $1.095 \pm 0.008$ \\
$5.0-5.5$ & $1.068 \pm 0.011$ \\
$5.5-6.0$ & $1.080 \pm 0.021$ \\
$6.0-6.5$ & $1.041 \pm 0.033$ \\
$6.5-7.0$ & $1.087 \pm 0.054$ \\
$7.0-7.5$ & $1.083 \pm 0.085$ \\
$7.5-8.5$ & $0.940 \pm 0.155$ \\
\hline
\end{tabular}

Table 6.3: Values of $\alpha$ averaged over the rapidity range $-0.75<y<0.75$.

bution functions were extracted from DIS and direct photon data. The pion fragmentation function was based on data from the one hadron inclusive production in $e^{+} e^{-}$and hadronic colliders as well as from selected fixed target experiments.

The theoretical cross sections were calculated for two energy scales $Q^{2}=p_{T}^{2}$ and $Q^{2}=p_{T}^{2} / 4$ over the c.m. rapidity range $-0.75<y<0.75$. $M_{d}^{2}$ and $M_{f}^{2}$ parameters defining distribution and fragmentation energy scales were assumed to be equal to $Q^{2}$. The results were multiplied by $A^{\alpha-1}$ to account for the observed A dependence of the cross section.

The comparisons for $\mathrm{Be}$ and $\mathrm{Cu}$ targets are presented in Figures 6.9 and 6.10 respectively. The agreement between the data and the theoretical predictions is good over a range of 6 orders of magnitude. The choice of $p_{T}^{2} / 4$ is clearly preferred for the energy scale.

\subsection{Major Sources of Systematic Uncertainties}

The major sources of the systematic uncertainties were: 


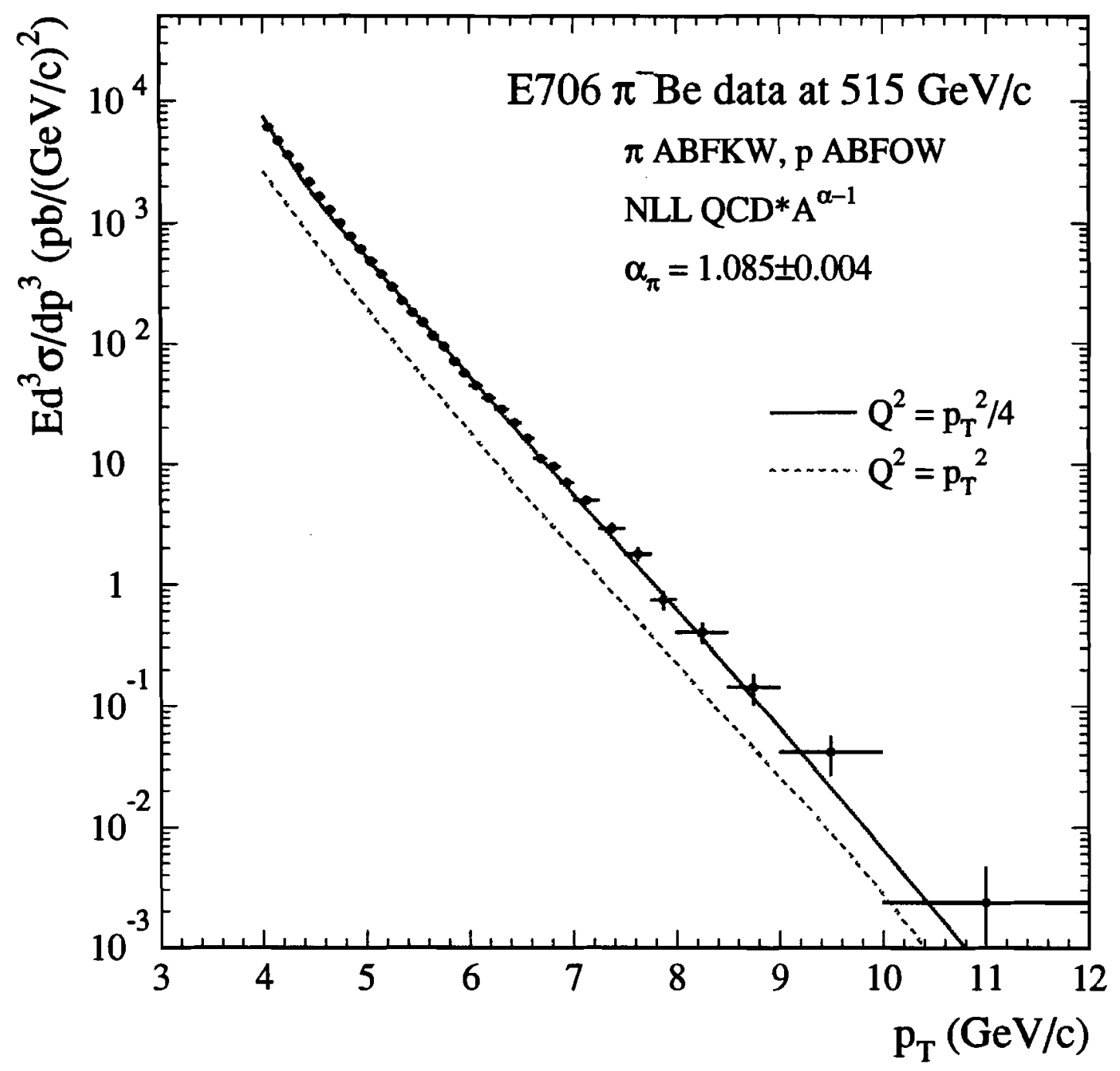

Figure 6.9: Comparison of the $\pi^{0}$ invariant cross section per nucleon on a Be target with the NLL calculations. 


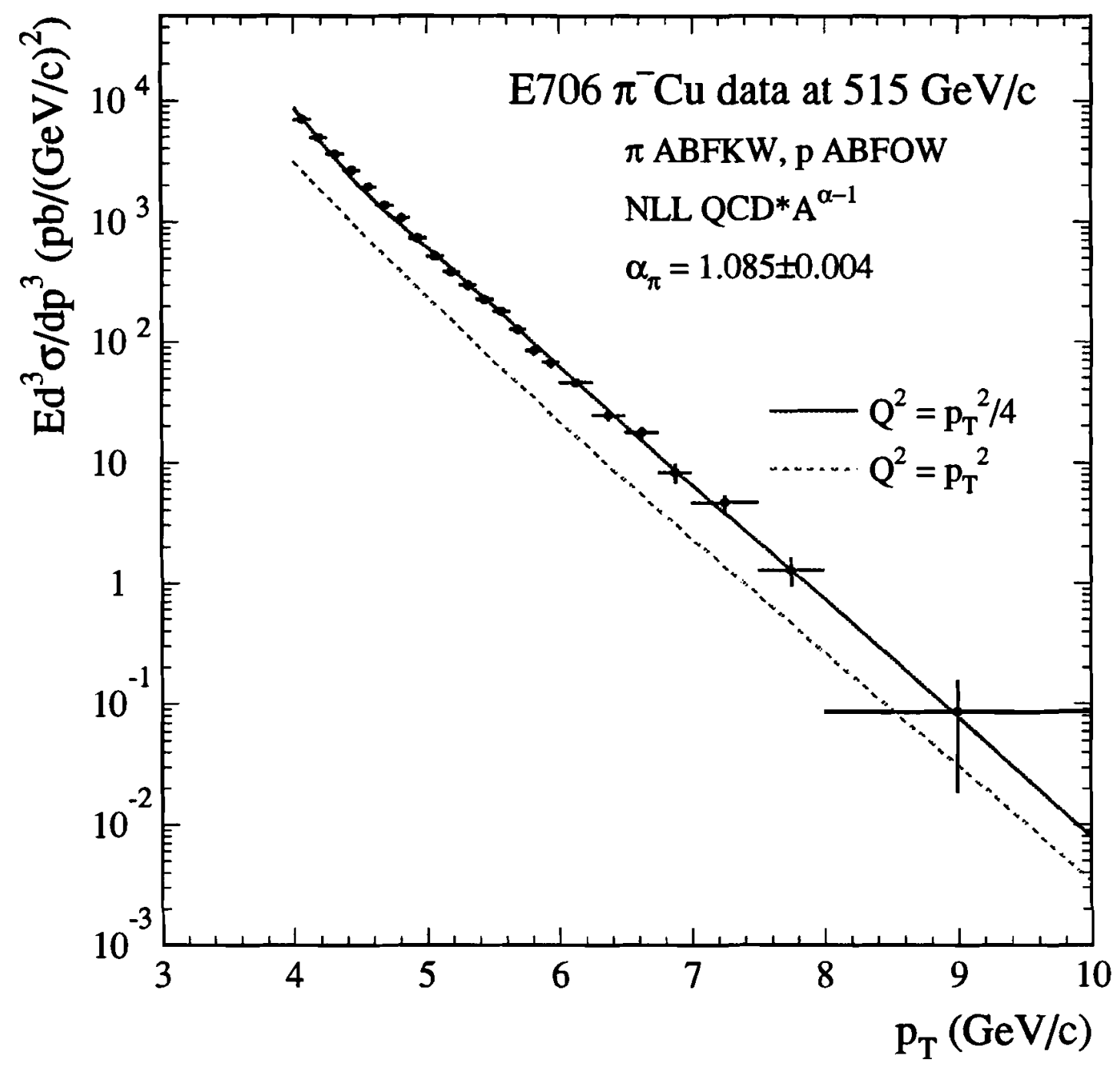

Figure 6.10: Comparison of the $\pi^{0}$ invariant cross section per nucleon on a $\mathrm{Cu}$ target with the NLL calculations. 
- the EMLAC energy scale: The energy scale of the EMLAC was determined using samples of $\pi^{0}$ and $\eta$ mesons in the two photon decay mode. The uncertainty was estimated to be $0.5 \%$ based on analysis of meson masses as functions of EMLAC octant, meson energy, meson position and energy asymmetry. Using Equation 6.1, we estimate the contribution to the cross section uncertainty to be $6 \%$ at $p_{T}=4.0 \mathrm{GeV} / \mathrm{c}$ and $7.5 \%$ at $p_{T}=8.0 \mathrm{GeV} / \mathrm{c}$.

- the $\pi^{0}$ reconstruction efficiency: The efficiency was obtained by fitting a smooth surface to the distribution obtained from MC data. The width of the residual distribution gave the estimate of the systematic uncertainty to be $5 \%$ for $p_{T}<6 \mathrm{GeV} / \mathrm{c}$ and $8 \%$ for $p_{T}>6 \mathrm{GeV} / \mathrm{c}$.

- the MC energy scale: Its uncertainty contributed an additional $6 \%$ to the systematic error of the cross section.

- the overall normalization: An additional $10 \%$ uncertainty was caused by uncertainties in the determination of LTB, losses during the data acquisition and reconstruction processes.

- photon conversions: The current understanding of the material encountered by photons contributed an additional $10 \%$ to the systematic uncertainties. Efforts are underway to minimize this contribution.

- smaller uncertainties, of the order of $1 \%$, originated from the trigger corrections, muon rejection cuts and the $\pi^{0}$ signal definition.

When added in quadrature, they contributed $17 \%$ systematic error to the measured cross section. 


\subsection{Conclusions}

This thesis reports on the production of $\pi^{0}$ mesons by a $\pi^{-}$beam on nuclear targets as studied with the MWEST spectrometer located at Fermilab. The apparatus consisted of a finely segmented lead liquid argon calorimeter and a sophisticated charged particle tracking system. The experiment utilized a $515 \mathrm{GeV} / \mathrm{c}$ secondary $\pi^{-}$beam and $\mathrm{Be}$ and $\mathrm{Cu}$ targets. The experiment triggered on events containing high $p_{T}$ electromagnetic showers detected in the calorimeter.

The inclusive $\pi^{0}$ cross sections were measured at $\sqrt{s}=31.1 \mathrm{GeV}$. Results were reported in the $4.0<p_{T}<12.0 \mathrm{GeV} / \mathrm{c}$ and $-0.75<y<0.75$ kinematic range for $\pi^{-}$Be interactions, and in the $4.0<p_{T}<10.0 \mathrm{GeV} / \mathrm{c}$ and $-0.75<y<0.75$ range for $\pi^{-} \mathrm{Cu}$ interactions. The cross sections fall as $p_{T}^{-n}$ with $n \sim 9.5$.

The values of the parameter $\alpha$ that describes the nuclear dependence of the cross section, and is defined by:

$$
\sigma_{A}=\sigma A=\sigma_{0} A^{\alpha}
$$

where $\mathrm{A}$ is atomic number of the target material, $\sigma$ is the cross section per nucleon and $\sigma_{0}$ is a constant, has been extracted from our data. Its value was determined to be $1.085 \pm 0.004$, and no dependence on $p_{T}$ and $y$ over the range $4.0<p_{T}<8.5$ $\mathrm{GeV} / \mathrm{c}$ and $-0.75<y<0.75$ was observed. The value of $\alpha$ is in agreement with measurements performed for charged pions.

Experimental cross sections were compared with an available next-to-leading logarithm PQCD calculation. The theoretical results were corrected for the A dependence using our measured dependence. Good agreement over six orders of magnitude in the cross section was found for the choice of $p_{T}^{2} / 4$ for the energy scale. 


\section{Bibliography}

-[1] W.M. Geist et al., Phys. Rep. 197 (1990), 263.

[2] T. Ferbel, W.R. Molzon, Rev. Mod. Phys. 56 (1984), 181.

[3] G. Alverson et al., Phys. Rev. Lett. 68 (1992), 2584.

[4] G. Alverson et al., Phys. Rev. D45 (1992), 3899.

[5] G. Alverson et al., Phys. Rev. D48 (1993), 5.

[6] G. Alverson et al., Phys. Rev. D49 (1993), 3106.

[7] H. Fritzsch et al., Phys. Lett. B47 (1973), 365.

[8] R. D. Field, Applications of Perturbative QCD, (1989).

[9] Review of Particle Properties, Phys. Lett. B239 (1992).

[10] D. Perkins, Introduction to High Energy Physics Ch.5, (1987).

[11] D.J. Gross, F. Wilczek, Phys. Rev. Lett. 30 (1973), 1343.

[12] H.D. Politzer, Phys. Rev. Lett. 30 (1973), 1346.

[13] S. Bethke, J.E. Pilcher, Ann. Rev Nucl. Part. Sci. 42 (1992), 251.

[14] A.S. Kronfeld, P.B. Mackenzie, Ann. Rev. Nucl. Part. Sci. 43 (1993), 793.

[15] J.F. Owens, Rev. Mod. Phys. 59 (1987), 465. 
[16] W.K. Tung, J.F. Owens, Ann. Rev. Nucl. Part. Sci 42 (1992), 291.

[17] K. Charchula at al., preprint DESY 90-019, (1990).

[18] R. Barlow, Rep. Prog. Phys. 56 (1993), N9.

[19] F. Aversa et al., Nucl. Phys. B327 (1989), 105.

[20] W. Giele et al., preprint FERMILAB - CONF - 91/243-T (1991).

[21] P. Aurenche et al., Phys. Lett. B233 (1989), 517.

[22] P. Aurenche et al., Phys. Rev. D39 (1989), 3275.

[23] P. Chiappetta et al., Nucl. Phys. B412 (1994), 3.

[24] G. Altarelli, G. Parisi, Nucl. Phys. B126 (1977), 298.

[25] J.W. Cronin et al., Phys. Rev. D11 (1975), 3105.

[26] G. Donaldson et al., Phys. Rev. Lett 36 (1976), 1110.

[27] H.J. Frisch et al., Phys. Rev. D27 (1983), 1001.

[28] L.R. Fortney, Proceedings DPF Meeting, Vancouver 1991, 669.

[29] J. Badier et al., Z. Phys. C30 (1986), 45.

[30] C. De Marzo et al., Phys. Rev. D36 (1987), 16.

[31] M. Bonesini et al., Z. Phys. C37 (1987), 39.

[32] I. Kourbanis, The A Dependence of Leading Particle Production by $800 \mathrm{GeV} / \mathrm{c}$ Protons, Ph. D. Dissertation, Northeastern University (1989).

[33] E. Engels et al., Nucl. Instr. Meth. A279 (1989), 272. 
[34] K. Hartman, Hadronic Production of $\pi^{0}$ Pairs and Associated Event Structure, $\mathrm{Ph}$. D. Dissertation, Pennsylvania State University (1990).

[35] C. Bromberg et al., Nucl. Instr. Meth A307 (1991), 292.

[36] F. Lobkowicz et al., Nucl. Instr. Meth. A235 (1985), 332.

[37] W. E. DeSoi, Construction and Performance of a Liquid Argon Calorimeter for Use in Experiment E-706 at the Fermi National Accelerator Laboratory, $\mathrm{Ph}$. D. Dissertation, University of Rochester (1990).

[38] C. Lirakis, A Study of High Transverse Momentum $\eta$ Production in $530 \mathrm{GeV} / \mathrm{c}$ Hadronic Interactions, Ph. D. Dissertation, Northeastern University (1990).

[39] R. Benson, Characteristics of Forward Energy Production in Proton - Nucleus and Pion - Nucleus Collisions at $\sqrt{ } s=31.5 \mathrm{GeV}, \mathrm{Ph}$. D. Dissertation, University of Minnesota (1990).

[40] VAXONLINE System, FNAL Computer Department PN-252.

[41] IEEE Standard FASTBUS, (1985), ISBN 471-84472-1.

[42] D.D. Skow, A Study of High Transverse Momentum Eta Meson Production, $\mathrm{Ph}$. D. Dissertation, University of Rochester (1990).

[43] G. Drake, Nucl. Instr. Meth. A269 (1988), 68.

[44] 1990 Research Instrumentation Catalog, LeCroy Corp.

[45] GPM Manual, STR500, Dr. B. Struck.

[46] C. Lirakis, Proceedings of the First Annual Conference on Electronics for Future Colliders, LeCroy Corp. (1991), 37. 
[47] DSP56000/DSP56001 Digital Signal Processor User's Manual, Motorola.

[48] C. Yosef, Production of High Transverse Momentum $\pi^{0}$ Mesons in Interactions of $530 \mathrm{GeV} / \mathrm{c}$ Proton and $\pi^{-}$Beams on Beryllium and Copper Targets, $\mathrm{Ph}$. D. Dissertation, Northeastern University (1990).

[49] L. Sorrell, private communication.

[50] N. Varelas, $\pi^{0}$ Production at High Transverse Momenta from $\pi^{-}$Collisions at $520 \mathrm{GeV} / \mathrm{c}$ on $\mathrm{Be}$ and $\mathrm{Cu}$ Targets, Ph. D. Dissertation, University of Rochester (1994).

[51] G.O. Alverson, E.L. Pothier, E706 Internal Note 139 (1985).

[52] H.J. Klein, J. Zoll, PATCHY Reference Manual, CERN (1983).

[53] R. Brun et al., ZEBRA User's Guide, CERN DD/EE/85-6.

[54] S. Blusk, private communication.

[55] J. P. Mansour ,High Transverse Momentum $\pi^{0}$ Production from $\pi^{-}$and $p$ Beams at $530 \mathrm{GeV} / \mathrm{c}$ on $\mathrm{Be}$ and $\mathrm{Cu}, \mathrm{Ph}$. D. Dissertation, University of Rochester (1989).

[56] A. Sinanidis, Particles Produced in Association with High Transverse Momentum Single Photon and $\pi^{0}$ in Hadronic Collisions, Ph. D. Dissertation, Northeastern University (1989).

[57] R.M. Roser, Eta Production at High Transverse Mdmentum by Negative 520 GeV/c Pions Incident on Beryllium and Copper Targets, Ph. D. Dissertation, University of Rochester (1994).

[58] G. Osborne, private communication. 
[59] M. Zieliński, private communication.

[60] L. Apanasevich, private communication.

[61] A. Carroll et al., Phys. Lett. B80 (1979), 319.

[62] J. Ftacnik, private communication.

[63] V. Zutshi, private communication.

[64] G. Marchesini et al., HERWIG V5.6, CERN (1993).

[65] H.U. Bengtsson, T. Sjostrand, Comp. Phys. Comm 46 (1987), 43.

[66] R. Brun et al., GEANT3 User's Guide, CERN DD/EE/84-1.

[67] P. Chang, private communication.

[68] G. Ginther, private communication.

[69] R.J. Miller, E706 Internal Note (1994).

[70] G. Osborne, E706 Internal Note 197. 


\section{RESUME}

\section{EDUCATION}

1994 Ph.D. in Physics NORTHEASTERN UNIVERSITY Boston, MA.

Thesis: "The Production of High $\mathrm{P}_{\mathrm{T}} \pi^{0}$ Mesons

in $515 \mathrm{GeV} / \mathrm{c} \pi^{-}-$Nucleon Collisions"

1990 M.S. in Physics NORTHEASTERN UNIVERSITY Boston, MA.

1983 M.S. in Technical Physics

UNIVERSITY OF MINING AND METALLURGY Krakow, POLAND.

\section{EXPERIENCE}

1989-1994 Research Assistant for Northeastern University

in residence at Fermi National Accelerator Laboratory, Batavia, IL.

- Involved in data analysis stage of fixed target direct photon experiment E706 which employed liquid argon electromagnetic calorimeter as a shower detector and trigger.

- Responsible for upgrades to code used to reconstruct electromagnetic showers.

- Participated in data collection and monitoring during two runs of E706.

- Worked on tuning of the beam for experiment - primary $(800 \mathrm{GeV} / \mathrm{c})$ proton beam and secondary beams of both polarities.

- Attended (for academic credit) 3 courses at US Particle Accelerator School.

- Wrote major portion of the liquid argon calorimeter readout software involving Motorola DSP56001 assembly language in FASTBUS environment.

1987-1989 Teaching/Research Assistant at Northeastern University.

- Worked on the first principles calculations of electronic structure of high- $T_{c}$ superconductors and transition metal alloys in Green's function formalism.

- Taught undergraduate physics laboratory.

1983-1987 Teaching/Research Assistant at University of Mining and Metallurgy.

- Worked on the calculations of the electronic structure of metals.

- Taught physics laboratory, conducted physics recitation sessions. 


$$
\begin{aligned}
& \text { H. } \quad(k+x)
\end{aligned}
$$

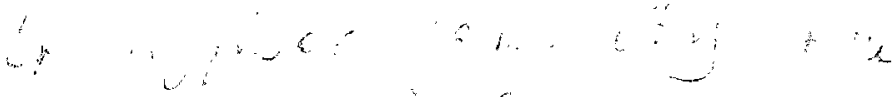

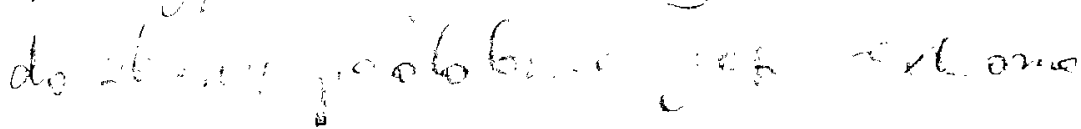

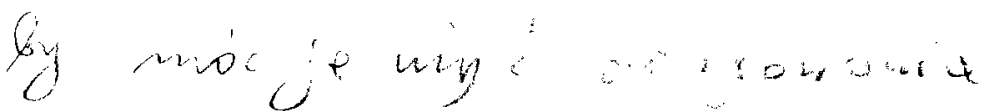

ivgrgire sumt

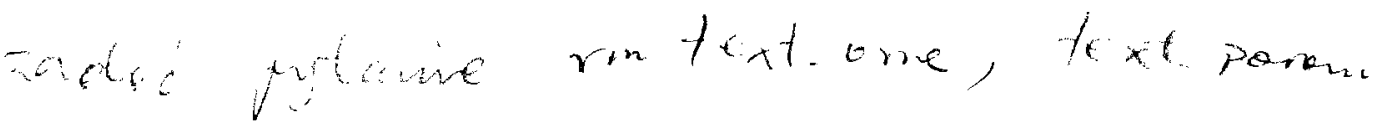

$i$ goly nie $\rightarrow$ axit

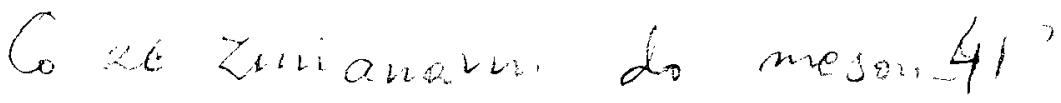

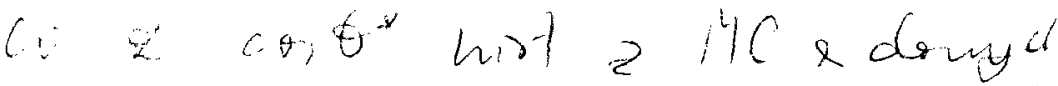

$$
\begin{aligned}
& z \text {. mositovinght? }
\end{aligned}
$$

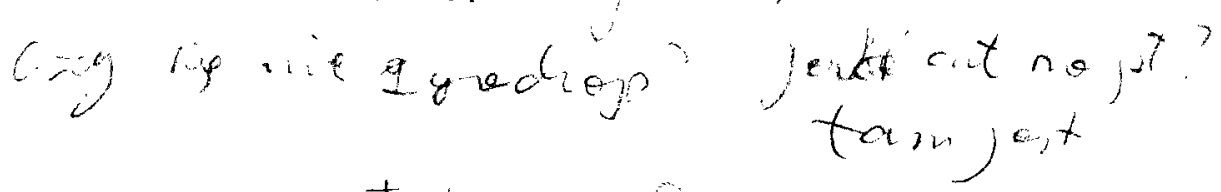

$$
\begin{aligned}
& A=\text { mon... trug gab., } \\
& \text { 1... b. by roatle" } S \angle H
\end{aligned}
$$

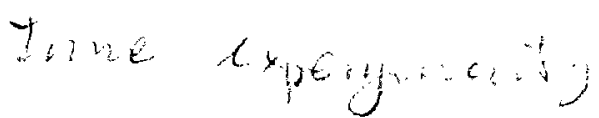

$$
\begin{aligned}
& C G H
\end{aligned}
$$

